\title{
Development of nanofluidic/microfluidic interfaces as analyte concentrators for proteomic samples
}

\author{
Kathleen C. Reschke \\ West Virginia University
}

Follow this and additional works at: https://researchrepository.wvu.edu/etd

\section{Recommended Citation}

Reschke, Kathleen C., "Development of nanofluidic/microfluidic interfaces as analyte concentrators for proteomic samples" (2010). Graduate Theses, Dissertations, and Problem Reports. 4646.

https://researchrepository.wvu.edu/etd/4646

This Dissertation is protected by copyright and/or related rights. It has been brought to you by the The Research Repository @ WVU with permission from the rights-holder(s). You are free to use this Dissertation in any way that is permitted by the copyright and related rights legislation that applies to your use. For other uses you must obtain permission from the rights-holder(s) directly, unless additional rights are indicated by a Creative Commons license in the record and/ or on the work itself. This Dissertation has been accepted for inclusion in WVU Graduate Theses, Dissertations, and Problem Reports collection by an authorized administrator of The Research Repository @ WVU.

For more information, please contact researchrepository@mail.wvu.edu. 


\title{
Development of Nanofluidic/Microfluidic Interfaces as Analyte Concentrators for Proteomic Samples
}

\author{
Kathleen C. Reschke \\ Dissertation submitted to the \\ Eberly College of Arts and Sciences \\ at West Virginia University \\ in partial fulfillment of the requirements \\ for the degree of \\ Doctor of Philosophy \\ in \\ Chemistry
}

Aaron T. Timperman, PhD., Chair

Ronald B. Smart, Ph.D.

Suzanne C. Bell, Ph.D.

Xiaodong Michael Shi, Ph.D.

Lisa M. Salati, Ph.D.

C. Eugene Bennett Department of Chemistry

Morgantown, West Virginia

2010

Keywords: Microfluidic devices, Proteomics, Nanocapillary membranes, Nanofluidics, Nanofluidic microfluidic interfaces, Concentration polarization, Current rectification 


\title{
Abstract \\ Development of Nanofluidic Microfluidic Interfaces as Analyte Concentrators for Proteomic Samples \\ Kathleen C. Reschke
}

\begin{abstract}
We have developed a nanofluidic microfluidic analyte concentrator for application to proteomic samples. This dissertation includes a study investigating the current rectification behavior of the device including a temporal characterization of the ON state to OFF state transition and the OFF state to ON state transition as a function of device design. An analyte concentrator compatible with proteomic samples is presented and the current rectifying phenomenon is utilized to ensure adequate analyte delivery for the enrichment process. Finally, characterization of a coating technique to alter the surface charge of the nanofluidic element from anionic to cationic is presented.
\end{abstract}




\section{Acknowledgements}

I would first like to thank my mentor, Dr. Corbin, from Ashland University. I did undergraduate research studying capillary electrophoresis with her and she encouraged me to pursue a position in a Research Experience for Undergraduates (REU) program. I spent 10 weeks at North Carolina State University the summer between my junior and senior year of college and had one of the best summers of my life. With her guidance, I applied for graduate school to study analytical chemistry and without her help I may not have attended graduate school.

I would like to acknowledge my advisor, Dr. Timperman, for his help throughout this journey. He provided a great variety of instrumentation that was useful to tackle obstacles along the research path and always found a way to provide the necessary funding for my research. He helped me to become a more confident and independent researcher. I would also like to thank my graduate committee for their guidance.

I would like to thank several different group members throughout the years. I would especially like to thank Dr. Matt Powell for his help in preparing for my proposal defense and bits of guidance he has passed along. I owe Dr. Trust Razunguzwa a special thank you for showing me how to use multiple pieces of instrumentation, discussing research problems, and helping me prepare for my proposal. I owe a great deal to Dr. Scott Miller who taught me all he knows about nanocapillary membranes and interfacing them to microfluidic devices. Jim Lenke always helped open my mind to the many possible ways to tackle a problem and taught me how to drill holes in microfluidic devices and offered technical expertise. I would also like to thank Brent Reschke, Ting Zhao, Heather Glover, Han Wang, Dr. Xiuli Mao, Dr. Yuchen Lu, Dr. Kyoo Jo, and Dr. Callee Walsh. Don Feathers, Allen Burns, Randy Eaglen, Philip Tucker, and Sherman Adams all contributed significant aspects to this work and without them I would not have been able to complete my research projects. 
Finally, I would like to thank my family for always being supportive of my decisions. I would like to thank my Dad for being the first person to show me how interesting and fun science is and my Mom for always being there to listen when things became difficult. Lastly but not least, I would like to thank my husband, Brent, for the supportive words that I could not have endured graduate school without. 


\section{Dedication}

To my loving and understanding husband Brent 


\section{Contents}

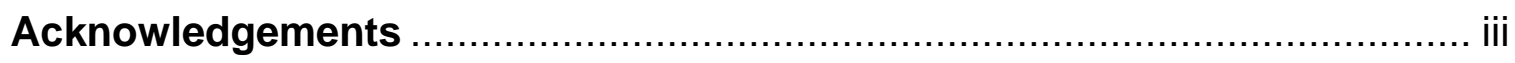

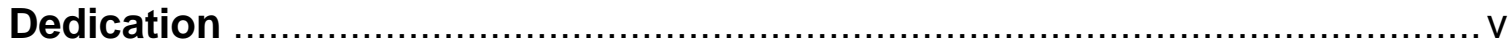

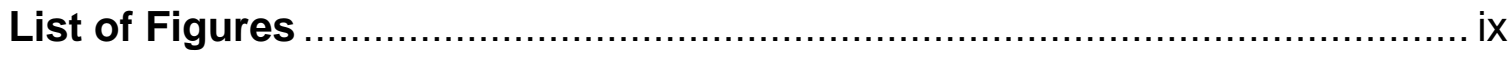

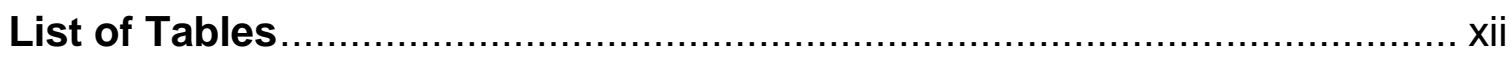

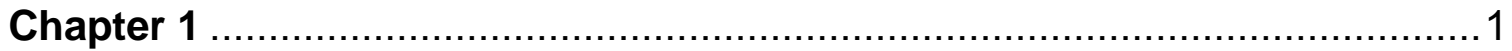

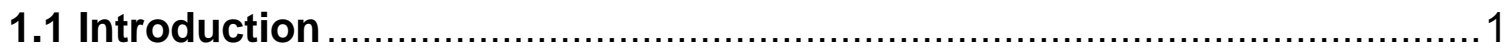

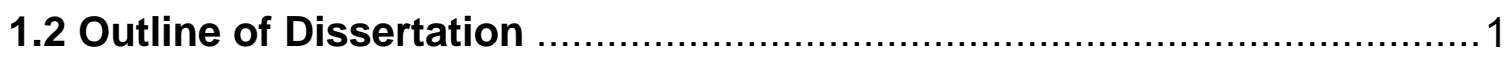

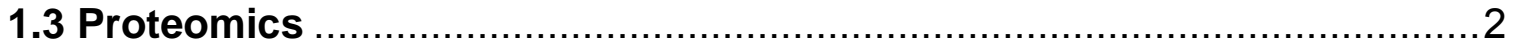

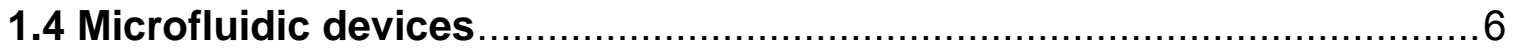

1.4.1 Device Fabrication ................................................................................... 10

1.4.2 Microfluidic Channel Fabrication......................................................... 11

1.4.3 Nanofluidic and Nanocapillary Membrane Fabrication ...................... 12

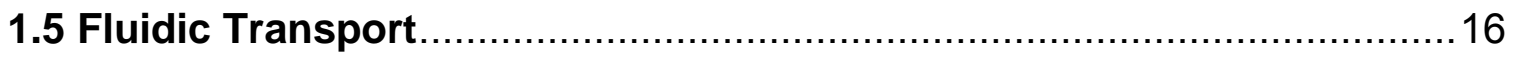

1.5.1 Transport through Microfluidic Channels ……..................................... 16

1.5.2 Transport through Nanofluidic Channels/Capillaries ......................... 19

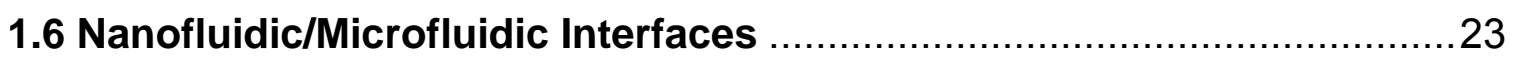

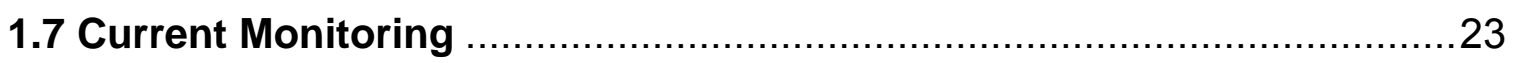

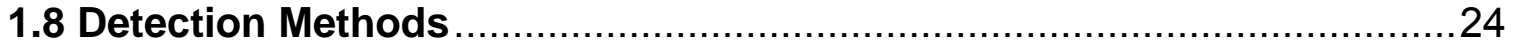

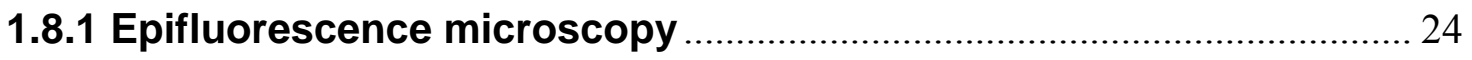

1.9 Proteomic Sample Analyses with Microfluidic Platforms …................26

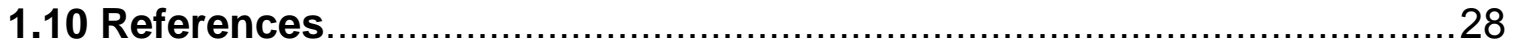

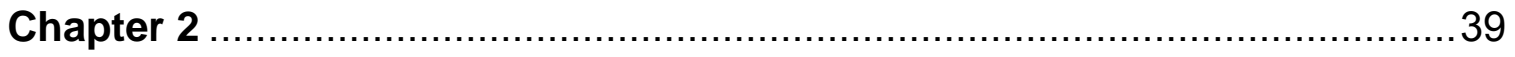

Ionic Current Rectification at a Nanofluidic/Microfluidic Interface with an

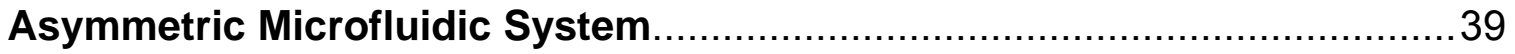

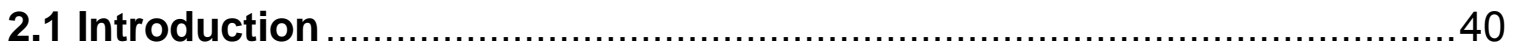

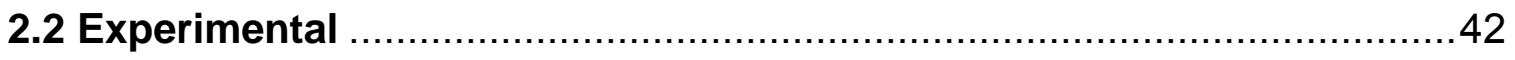

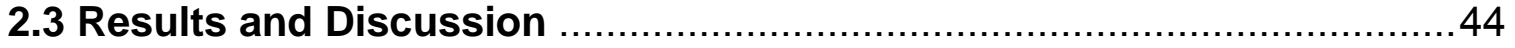

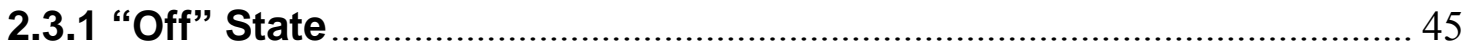

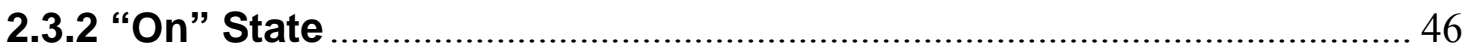




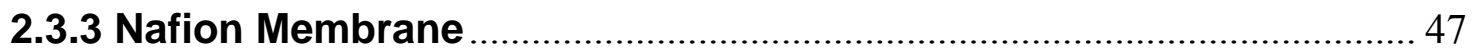

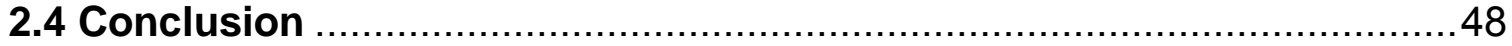

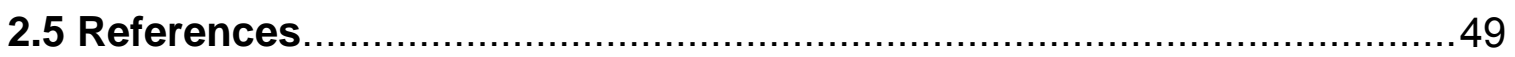

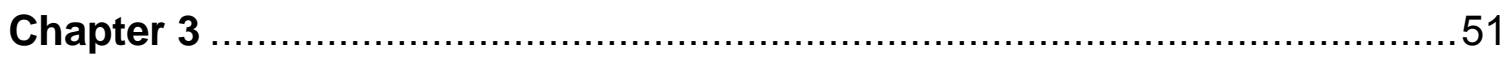

Investigation of Zone Migration in a Current Rectifying Nanofluidic/Microfluidic Analyte Concentrator .....................................51

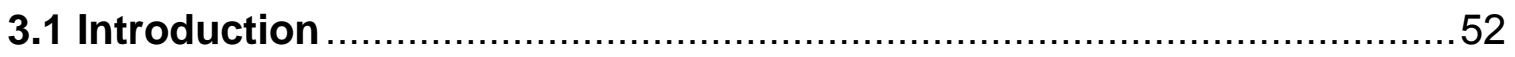

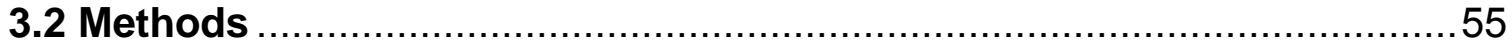

3.2.1 Fabrication of Glass Microfluidic Devices ........................................... 55

3.2.2 Integration of NCMs and Device Preparation ....................................... 57

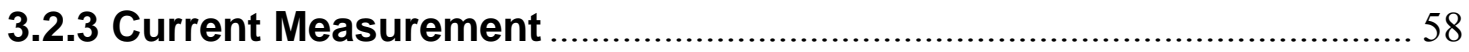

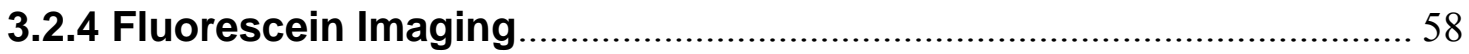

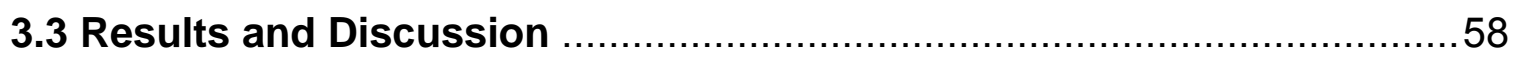

3.3.1 Changing Volume at the NCM/Microfluidic Channel Interface .......... 59

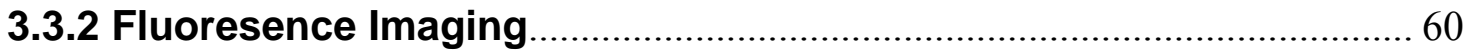

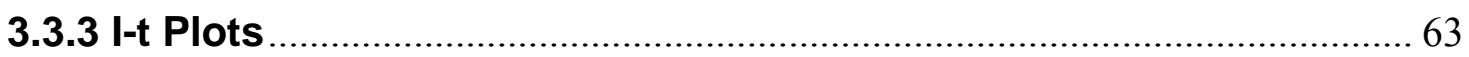

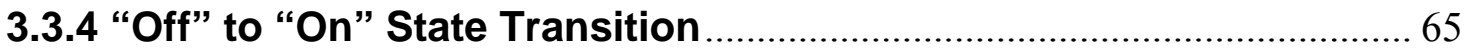

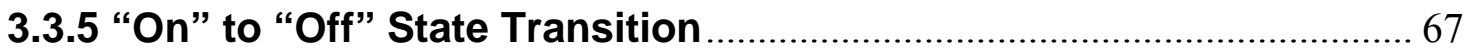

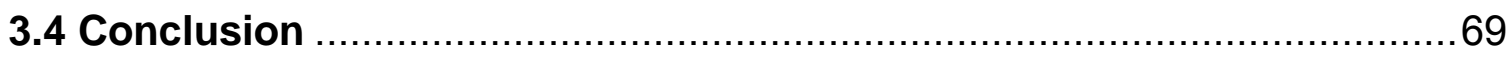

3.5 References

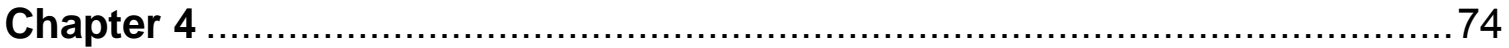

Development of an ON State Electrophoretic Dominant Concentrator Based on a Nanofluidic/Microfluidic Interface ................................................. 74

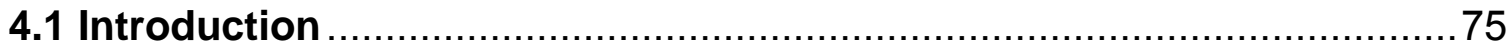

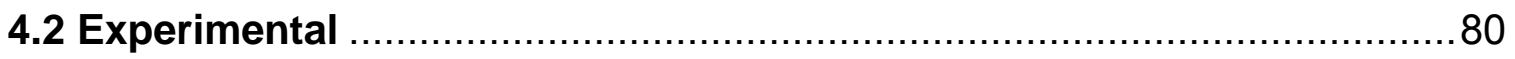

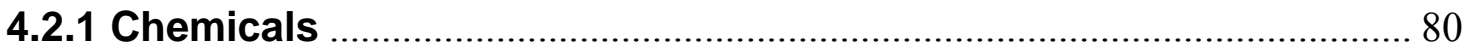

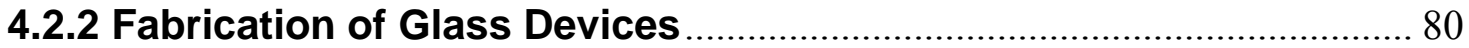

4.2.3 Formation and Measurement of the Nanochannel................................ 81

4.2.4 Fabrication of Hybrid PDMS-Glass Devices ………………………..... 83

4.2.5 Enrichment Process and Epifluorescence Imaging …….................... 85

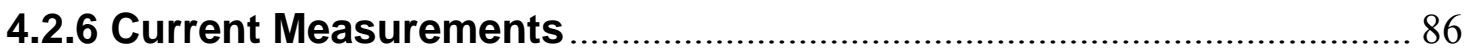




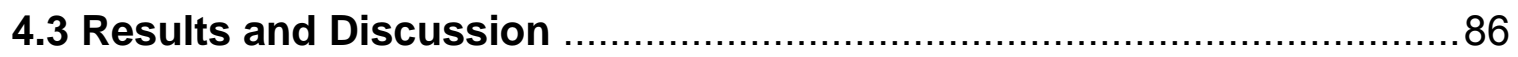

4.3.1 Measurement of Nanochannel Height ................................................ 86

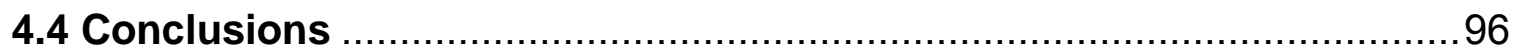

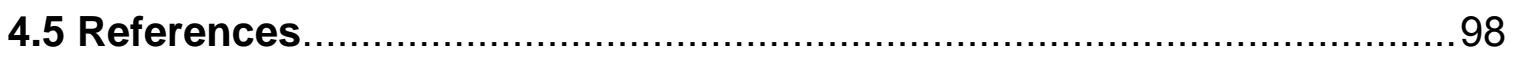

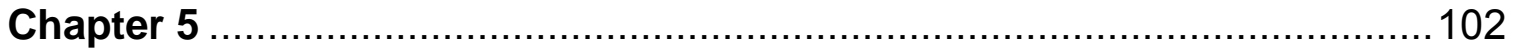

Positively Coated Nuclear Track Etched Nanocapillary Membranes for

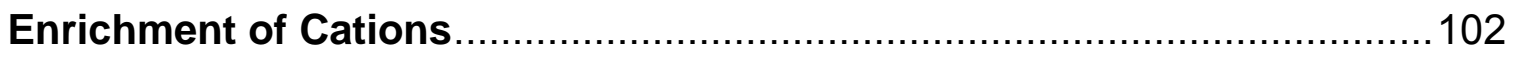

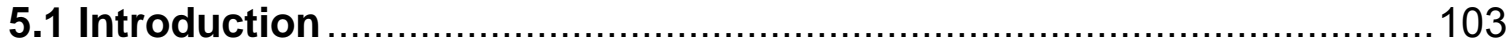

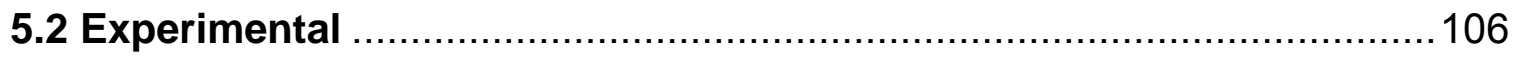

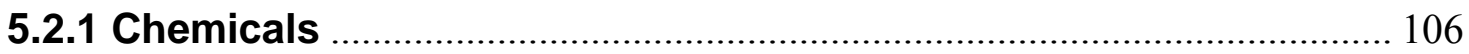

5.2.2 NCM Preparation and Coating .......................................................... 106

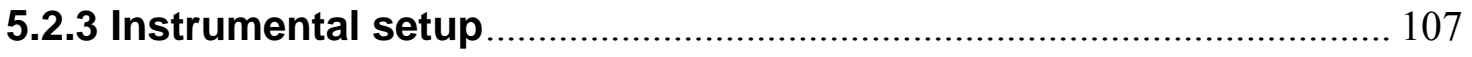

5.2.4 Calibration Curve Determination ........................................................... 109

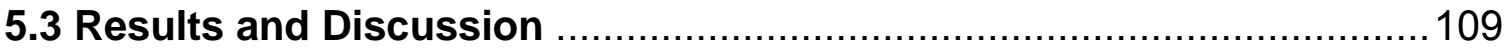

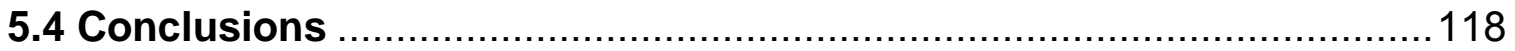

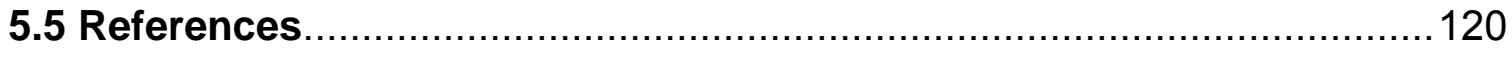

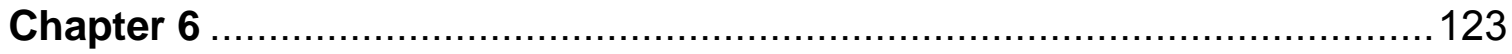

Future Work 


\section{List of Figures}

Figure 1.1: Schematic of the wet chemical etching process for glass microfluidic devices.

Figure 1.2: Schematic illustrating the parabolic flow profile of hydrodynamic flow in a microfluidic device.

Figure 1.3: Schematic illustrating the double layer associated with a negative surface. 18

Figure 1.4: Schematic illustrating the flat plug profile of EOF

Figure 1.5: Schematic illustrating double layer overlap in negatively charged nanochannels or nanocapillaries at varying buffer concentrations.

Figure 1.6: Schematic of concentration polarization. ........................................... 23

Figure 1.7: Schematic of an Epifluorescence system .......................................... 26

Figure 2.1: Schematic of NMI in which the NCM connects a microfluidic channel and macroscopic NCM reservoir.

Figure 2.2: I-V curve in which the average currents are plotted as a function of potential applied across the microchannel interface (red) with a NCM and (blue) without a NCM. 45

Figure 2.3: I-V curve in which the average current was plotted as a function of potential applied across the microchannel side-interfaced (blue) with a Nafion-117 proton exchange membrane and (red) without a membrane.. 47

Figure 3 1: Three device designs are shown for the NMI with the PC-NCM.... 56

Figure 3.2: Images of the enriched zone of fluorescein in the microchannel of the device design shown in Figure 3.1A..

Figure 3.3: Fluorescent images showing the elution of an enriched zone of fluorescein in an NMI with the alumina backed side interface design (Figure 3.1C).

Figure 3.4: The current responses as a function of time are shown for the corresponding device designs shown in Figure 3.1A, 3.1B, and $3.1 \mathrm{C}$ as each device is switched between the "on" and the "off" states, by applying $+800 \mathrm{~V}$ and $-800 \mathrm{~V}$ at the NCM reservoir respectively. 
Figure 3.5: A schematic representation of the enriched zone and CP zones following the "off" to "on" state transition..

Figure 3.6: A schematic representation of the "on" to "off" state transition is shown.

Figure 4.1: Schematic representation of the location of CP zones according to the applied voltage and location of enriched anionic sample in both high EOF and low EOF devices..

Figure 4.2: Schematics of device designs A) Schematic of symmetric glass microfluidic device.

Figure 4 3: A) A representative micrograph acquired with a 10x objective of the microchannels and the nanochannel formed using dielectric breakdown..

Figure 4.4: The symmetric device design is used to obtain the data presented and only one v-shaped channel was utilized to acquire the data. 88

Figure 4.5: The average intensity vs. time is presented for the symmetric device.

Figure 4.6: Current vs. voltage plots for the symmetric device.. 91

Figure 4.7: Asymmetric device is used to collect the data presented.. 93

Figure 4.8: Current vs. time plot that corresponds to the fluorescence imaging in Figure 4.7.. 95

Figure 4.9: The average intensity vs. time is presented for the asymmetric device...

Figure 5.1: Schematic representation of the experimental setup.

Figure 5.2: Calibration curve for the fluorescence produced from negative fluorescein (blue diamonds), positive rhodamine 123 (red squares), and neutral riboflavin (green triangles) at given concentrations

Figure 5 3: Representative transport of a neutral marker, riboflavin, through uncoated (blue) and coated (orange) NCMs in response to applied currents 
Figure 5 4: Representative transport of a negative marker, fluorescein, through uncoated (blue) and coated (orange) NCMs in response to applied currents.

Figure 5 5: Representative transport of a positive marker, rhodamine 123, through uncoated (blue) and coated (orange) NCMs in response to applied currents. 


\section{List of Tables}

Table 5 1: Comparison of Electroosmotic Flow produced by Coated and

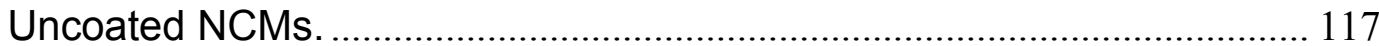




\section{Chapter 1}

\subsection{Introduction}

\subsection{Outline of Dissertation}

This dissertation investigates the characteristics and behavior of a nanofluidic/microfluidic interface (NMI) to aid in the development of an analyte concentrator for proteomic samples. The first chapter presents a basic overview of the components involved in the research projects while the following four chapters are detailed studies that contribute to the construction of an analyte concentrator for a lab on a chip device for proteomic analysis. Chapter 2 involves the characterization of a NMI for use as an analyte concentrator. This chapter reveals the current rectification phenomena associated with the analyte concentrator design through current versus voltage plots and helps to create an understanding of how the device operates. Chapter 3 investigates the current rectification of the analyte concentrator in further detail through current versus time plots. The current versus time plots along with corresponding images reveal how the device transitions from the off state, or low current, to the on state, or high current. Chapter two and three both show the analyte is delivered via electroosmotic flow (EOF) as it becomes enriched, which leads to analytes separating according to their electrophoretic mobility and requires the enrichment process to be tuned to the electrophoretic mobility of particular analytes. A more universal and compatible method of enriching proteomic samples would rely on electrophoresis to deliver the sample and in Chapter 4 a design is created using the knowledge of previous chapters to make a concentrator that delivers sample via electrophoresis. Prior to Chapter 5 , all the concentrators were only capable of enriching anions. However, it is also desirable to enrich both anionic and cationic analytes, as proteomic samples will likely contain both. Therefore, Chapter 5 develops a method to coat the nanocapillary membranes (NCM) to create a positive surface charge that will allow the NCM to enrich cations when properly interfaced with a microfluidic channel. 


\subsection{Proteomics}

Proteomics is a relatively new area of research and is a discipline of systems biology. Systems biology approaches biology by investigating the biological system rather than understanding the isolated parts of the biological system that may be incapable of explaining the behavior of the system as a whole. Therefore, proteomics has a similar approach and accordingly has the challenging goal of identifying all the proteins and their roles within the system. Proteomics differs greatly from protein chemistry in which the focus is placed on a single or a few proteins. In protein chemistry, a protein is sequenced in its entirety and its structure is determined. The structure of the protein is then used to identify the function of the particular protein within a given system. Proteomics is different in that only partial sequencing is performed, which was made possible through the human genome project and the determination of the genomes of other animals and organisms. ${ }^{1}$

The genome dictates the possible proteins that can be produced in a given organism through DNA that is housed in the nucleus of each cell or in a general region of prokaryotic cells. Within eukaryotic cells, the DNA is transcribed into RNA within the nucleus and minor changes are made to the RNA to modify it into messenger RNA (mRNA). The mRNA is then moved out of the nucleus into the cytosol and into ribosomes that translate the code of the mRNA into proteins. ${ }^{2}$ Therefore, the possible protein sequences can be directly correlated back and predicted from the DNA of an organism and the need to sequence all the peptides from a protein is unnecessary to identify a protein with statistical certainty. If a few peptides unique to a particular protein are sequenced, then the protein is identified with statistical certainty using the genetic information to first narrow the possible protein sequences and then identifying a combination of peptides unique to a predicted protein.

It may seem unnecessary to study the proteins and instead place the focus on the concentration and sequence of the mRNA. However, the levels of mRNA found in the cell do not directly correlate to the amount of protein 
produced because as in most processes several variables are at play. ${ }^{3}$ Different strands of mRNA can have different stabilities or different rates of translation into protein. The differences between strands of mRNA are not the only variable, because proteins can have post-translational modifications such as phosphorylation, glycosylation, methylation, or acetylation following translation. These modifications can influence the behavior of a protein by causing it to be more or less active. Furthermore, the mRNA does not offer any information about whether the protein will be modified post-translationally. The posttranslational modifications are carried out after the sequence of the mRNA has been translated into a sequence of amino acids or a protein and therefore the mRNA does not influence whether the protein becomes modified. Clearly, a complete understanding of the events within a cellular system cannot be deduced from only the mRNA, so a need to study the behavior and identity of other biologically relevant molecules within systems such as proteins arises. ${ }^{1}$ Therefore, it is important to have appropriate technologies to address the field of proteomics.

Proteomics is an area of research that is pushing current analytical instrumentation past its capabilities by introducing samples with a diverse range of solubilities, large dynamic range, and low concentrations. The diversity of the samples within the proteome is unusual. Proteins are a combination of typically 20 different amino acids that vary in hydrophobicity, polarity, and charge. In addition to these varied amino acids, proteins can be modified following translation meaning different versions of the same protein can exist depending on conditions following translation. The properties of proteins vary so greatly that it can be a challenge to keep them all solubilized and then separate them effectively.

Proteins can also be found at very different concentrations within the same cell. Some proteins may have as little as ten copies per cell while another protein within the same cell may have a million copies per cell. ${ }^{4}$ Within human serum samples, it is estimated that 20,000 different proteins are present with concentrations ranging over 10 orders of magnitude. ${ }^{5}$ Human serum samples 
are one of the most complex proteomic samples, but even yeast (Saccharomyces cerevisiae) cell lysates have been shown to express as many as 4,517 proteins over a dynamic range from fewer than 50 copies to $10^{6}$ copies of protein per cell. ${ }^{6}$ Therefore, it becomes difficult to detect both proteins of low abundance and proteins of high abundance. Typically, the high abundant proteins mask the signal from the low abundance proteins. Therefore, both the separation and concentration of the low abundance proteins is critical to their detection and methods to minimize sample loss and ensure low abundance proteins are concentrated enough must be employed. Two general approaches for proteomics research have been developed thus far and are referred to as bottom-up proteomics and top-down proteomics.

Bottom-up techniques first digest the proteins into peptides; next the peptides are separated and identified, and finally correlated back to the whole protein. Conversely, top-down techniques keep the proteins intact and separate and identify the protein as a whole. Bottom-up techniques are better suited for sequencing unknown proteins while top-down techniques are more useful for distinguishing differences between proteins such as post-translational modifications. Often multidimensional separations are employed to sufficiently separate samples from bottom-up techniques. A typical separation can involve techniques such as two-dimensional gel electrophoresis (2DE) followed by reversed-phase high performance liquid chromatography (RP-HPLC). When using $2 \mathrm{DE}$, the first dimension separates the proteins based on isoelectric point and the second dimension separates the proteins based on molecular weight. 2DE can typically only separate 1000 to 2000 different proteins. ${ }^{7}$ The protein bands are then excised and digested using an enzyme, most commonly trypsin, to make peptides that are much smaller and easier to sequence. Finally, the peptides are further separated using RP-HPLC and identified using mass spectrometry (MS). The solubility of the proteins can become problematic using these techniques since the proteins remain intact through the first two separation methods. The typical dynamic range of such methods are on the order of $10^{2}$ to 
$10^{4}$, which is far less than the dynamic range needed for the analysis of human serum samples and even yeast cell lysates. ${ }^{5}$

Another bottom-up approach is multidimensional protein identification technology (MudPIT), which is also referred to as shot-gun sequencing, in which a proteomic sample, containing many different proteins, is first digested. ${ }^{8}$ However, digesting the proteins into peptides complicates the sample even further. The digestion is followed by ion exchange chromatography followed by RP-HPLC and electrospray ionization (ESI)-MS for identification. Digestion of the proteomic sample prior to any separation allows for less concern about the solubility of the sample, because peptides are generally more soluble than proteins. ${ }^{9}$ However, there initially were many proteins and it is more difficult to determine the origin of any single peptide. To ease this difficulty, MS in combination with database searching is often employed for peptide and/or protein identification.

MS is a common theme in all proteomics approaches and has become an indispensable analytical instrument for proteomics due to its low limits of detection, ability to provide structural information, database matching capabilities, and the ease with which it can be directly coupled to separation techniques such as LC and capillary electrophoresis (CE). MS can be coupled to LC and CE techniques through soft ionization techniques that allow intact gas phase ions to be created from biomolecules for MS analysis. MS also has the ability to determine the masses of both proteins and peptides accurately. While the high accuracy mass determination of the proteins is often not successful for whole protein identification, it is highly useful in determining post-translational modifications. However, when a few peptides are identified with high mass accuracy, from fragmentation techniques, the identity of the protein can often be determined with the aid of database matching techniques.

While MS is an excellent detection method for proteomics, better sample preparation and separation techniques are still required to meet the challenges of proteomic samples, namely the sheer number of proteins present and the dynamic range of the samples. Separation methods with improved peak 
capacities will aid in making proteomics a feasible endeavor. Methods of developing separation techniques with improved peak capacities often involve coupling multiple separations, which improves the overall peak capacity significantly because it is the product of the individual separations' peak capacities.

In addition to better separation techniques, methods to couple separation techniques with minimal sample loss and contamination are needed for proteomic analysis. Sample loss and contamination are especially problematic when dealing with low abundant proteins, because they are more susceptible to masking making their separation and identification more difficult. Furthermore, the complete analysis of a proteomic sample can be very labor intensive and time consuming. Therefore, improved technology that minimizes sample analysis time and yields more information simultaneously is necessary.

\subsection{Microfluidic devices}

Microfluidics, or lab on a chip technology, offers a method to couple multiple separation techniques together with near zero dead volume intersections. The near zero dead volume intersections minimize band broadening from one separation technique to the next and require less sample and solvent compared to current techniques, an important feature when a limited amount of sample is available. In addition, microfluidics allows for multiple samples to be analyzed in parallel through several steps. Consequently, microfluidics has the potential to analyze the same sample using both bottom-up and top-down proteomics techniques simultaneously allowing both sequence and post-translational modification information to be gathered quickly.

Lab on a chip technology also minimizes sample contamination and loss due to the multiplexing capabilities that decrease the transfer and handling of the sample because each sample preparation step is contained within the microfluidic device. Sample contamination and loss is a key factor to proteomic samples where the concentrations are low at the start and a contamination may mask the signal of the analyte of interest. Microfluidic systems clearly offer many 
advantages for multiplexing separation techniques; however this ability can also be extended to processes such as sample enrichment as well.

Sample enrichment techniques are often a necessity when utilizing microfluidic devices for analyses. While microfluidic devices only consume a small volume of sample, a greater demand is placed on detection systems when only small amounts of sample are used. Several techniques for in solution concentration techniques have been developed for microfluidic devices including solid phase extraction (SPE), ${ }^{10-14}$ isoelectric focusing (IEF), ${ }^{15-20}$

isotachophoresis, ${ }^{21-25}$ field amplified stacking, ${ }^{26-28}$ field gradient methods, ${ }^{29-41}$ and nanofluidic preconcentrators. ${ }^{42-66}$

SPE is a technique in which sample is selectively bound to a stationary phase and then eluted with a buffer system different from the buffer originally used to dissolve the sample. SPE allows for a large volume of sample to be washed across the stationary phase where the analyte of interest is bound to the stationary phase and contaminants that do not interact with the stationary phase are rinsed away. The analyte is then eluted as a more concentrated solution with fewer contaminants in the sample. While SPE is effective, it can be difficult to integrate multiple buffer systems onto a microfluidic platform. Also, the performance of SPE is highly influenced by the properties of the analyte and therefore the efficacy is dependant upon the particular analyte. The recovery of analytes is dependent on their individual interactions with the stationary and mobile phases and therefore the amount of sample enrichment will vary from analyte to analyte.

IEF is widely applicable to protein samples. IEF has been performed both as the first stage of a gel and within microfluidic devices, but generally the principles of the method are the same regardless of whether it is performed as the first stage of a gel or within a microfluidic device. In IEF, a pH gradient is generated by using a dissimilar (high and low pH buffers) and an external electric field. Ampholytes, which is a suite of molecules with varying $\mathrm{pK}_{\mathrm{a}}$ values, are used to help stabilize the $\mathrm{pH}$ gradient. An acidic buffer is placed at the anode and a basic buffer is placed at the cathode. With the application of an electric 
field the hydronium and hydroxide ions cross-migrate and create a $\mathrm{pH}$ gradient. Analytes being focused migrate in this gradient until they reach their isoelectric point ( $\mathrm{pl}$ ) where they are neutral and no longer respond to the electric field. At their pl the analyte becomes stationary, which allows for analyte to become enriched in a small band. The ampholytes are focused in a similar manner and help mask the charge of the analyte molecules, so the charge of the analyte molecules has less of an effect on the local electric field as they are focused. However, for proteins, the $\mathrm{pl}$ is where they are most insoluble and therefore, methods of resolubilizing the proteins are sometimes necessary.

Another electrophoretic method called isotachophoresis exploits differences in buffer characteristics to enrich sample. Initially, a capillary is filled with buffer containing an electrolyte with a fast mobility (leading buffer) followed by the sample dissolved in water and finally followed by a buffer containing an electrolyte with a low mobility (terminating buffer). When a voltage is applied across the capillary, the leading buffer has a low electric field and the terminating buffer has a high electric field. As the sample migrates toward the outlet at a steady state velocity it becomes focused from the non-uniform electric field. The technique has minimal band broadening, because as analyte begins to lag behind the steady state migration velocity then the migration velocity of the analyte will increase as it reaches a zone with a greater electric field and the opposite occurs in the case of the analyte migrating faster than its steady state velocity. However, the buffers must be chosen appropriately to address the sample such that the mobility of the sample lies between the mobilities of the additives in the leading and terminating buffers. Also, the device is complicated by the need to introduce multiple buffer systems.

A similar method to isotachophoresis is field amplified stacking. However, field amplified stacking uses the same buffer throughout but varies the buffer concentration. The capillary is filled with a higher buffer concentration than the buffer concentration used to dissolve the sample. This causes the electric field in the sample plug to be high and the analyte quickly migrates to the buffer with higher concentration where the electric field is lower. As the sample reaches the 
more concentrated buffer it slows, and becomes focused. While this method does keep a uniform buffer composition, it still requires buffers with different concentrations.

Field gradient techniques generally involve balancing two flows against one another to result in enriched sample bands. Electric field gradient methods typically involve balancing bulk flow against an electric field gradient and analytes focus where the hydrodynamic flow equals their electrophoretic velocity. Electric field gradient methods, while effective for concentrating analyte, require the bulk flow used to enrich sample to be isolated from latter stages of analysis on the device. A related method, temperature gradient focusing, creates a gradient in the electrophoretic velocities of analytes by using a temperature gradient. ${ }^{30-34}$ The temperature gradient causes the viscosity of the buffer solution to vary in the adjacent buffer. When a potential is applied across the temperature gradient, the electrophoretic velocity of the analyte changes along the temperature gradient. Ultimately, when the velocity of the analyte is balanced by the bulk flow in the device, the analyte becomes enriched.

Finally, nanofluidic concentrators incorporate a nanofluidic element into the microfluidic device and an electric field is applied across the nanofluidic element to cause sample enrichment. Nanofluidic concentrators do not require complex buffer systems for successful operation and two modes of sample enrichment have been reported for nanofluidic concentrators: size exclusion and electrostatic exclusion enrichment. The size-based concentration methods involve media that pass small molecules, but large molecules such as DNA and proteins are retained. ${ }^{45-47,59,66-68}$ However, smaller analytes including peptides easily pass through the size-based selector. Thus, size-based methods are useful for whole protein work.

The electrostatic exclusion enrichment essentially uses the nanofluidic element to allow analyte of one charge to pass while the other remains and becomes enriched as more sample is delivered to the interface. ${ }^{42-44,48,50-52,54-58,60-}$ 65 The electrostatic exclusion enrichment relies on a permselective element (a media that preferentially transports either cations or anions). The permselective 
element could be a nonochannel, hydrogel, or similar material with small capillaries or pores and substantial surface charge. Therefore, both small and large molecules can be enriched and these types of concentrators are compatible with both bottom-up and top-down proteomic approaches. Furthermore, nanofluidic concentrators in electrostatic exclusion mode have some of the highest reported concentration factors and show much promise as they do not require complex buffer systems, are relatively universal, and are highly effective at enriching sample.

\subsubsection{Device Fabrication}

Different types of substrates have been used to create microfluidic devices such as glass, polydimethylsiloxane (PDMS), and polydimethylmethacrylate (PMMA) to list some of the most common. The type of substrate chosen varies with the application and all substrates have advantages and disadvantages. In general, plastic devices are cheaper and faster to produce than glass devices. However, plastic devices often suffer from incompatibility with organic solvents that cause them to swell and distort their shape causing leaks. In addition, plastics that are more resistant to solvents are typically not transparent making optical detection difficult. Glass devices do not have a solvent incompatibility problem, but they do have high electroosmotic flow natively resulting from a relatively high negative surface charge at $\mathrm{pH}>4$. The surface charge is also problematic and causes adsorption of sample to the surface leading to peak tailing and loss of sample. Samples that are positively charged tend to adsorb to the negative surface through electrostatic interactions. However, glass devices have well characterized surface chemistry and multiple coating methods are available to tailor the surface chemistry to the requirements of particular applications. Therefore, the surface can be altered to minimize interactions with the sample that will lead to degraded separations and sample loss. 


\subsubsection{Microfluidic Channel Fabrication}

Once the appropriate substrate for the application is selected, a series of channels must be fabricated to construct the microfluidic device.

Photolithography and wet chemical etching techniques are used to make the glass devices, which relies on photoresist deposited on a chrome layer that is deposited onto the glass surface. A pattern is transferred to the photoresist by exposure to ultraviolet (UV) light through distinct regions defined by a photomask with transparent areas to define channels as shown in Figure 1.1. Once exposed to the UV light, depending on the type of photoresist, the bonds will either be broken or formed.

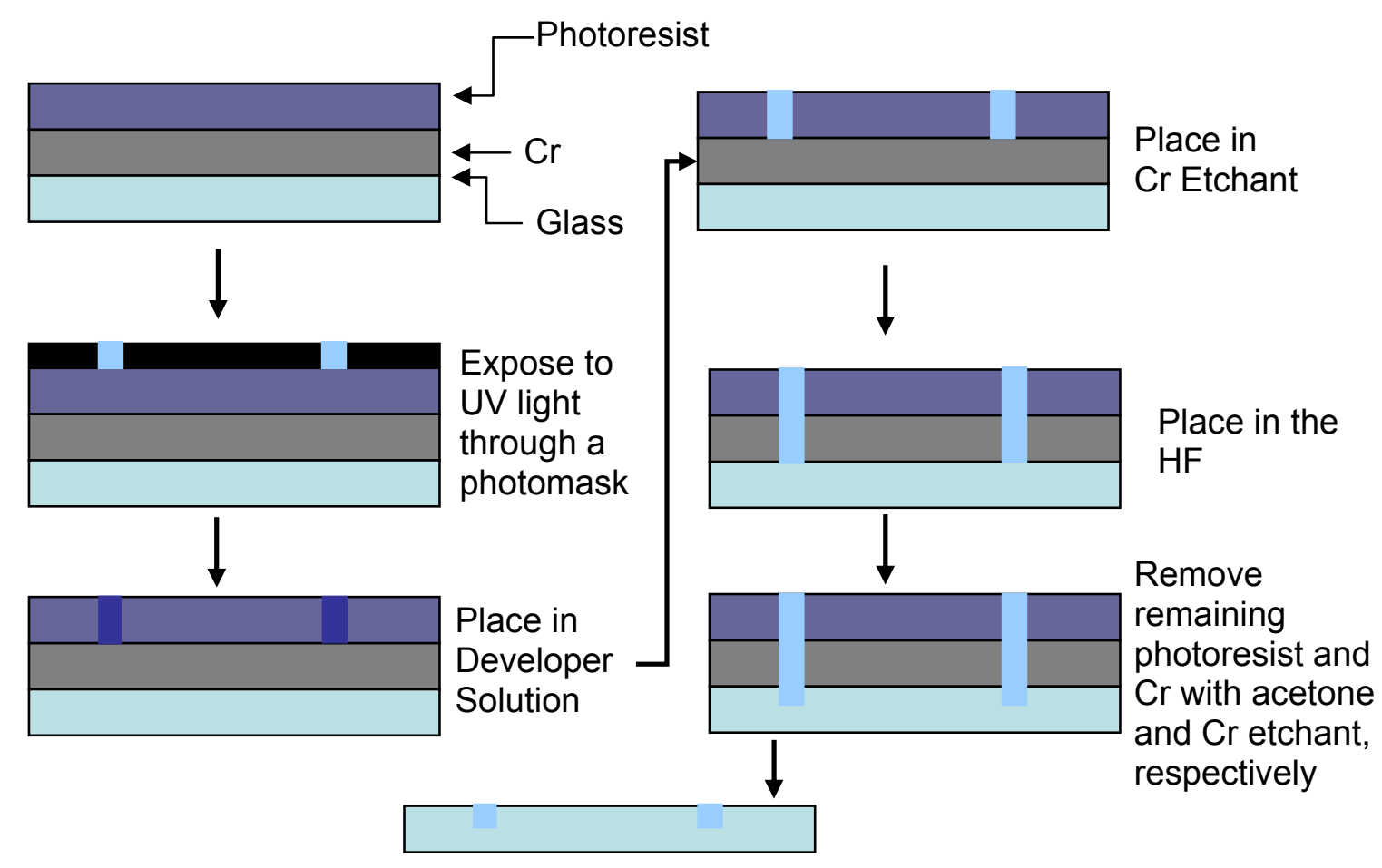

Glass wafer with microchannels

Figure 1.1: Schematic of the wet chemical etching process for glass microfluidic devices.

For glass devices, the photoresist is typically a positive photoresist meaning the bonds are broken when exposed to UV light allowing this region of the photoresist to be soluble in the photoresist developer. Once placed in the developer solution, the degraded photoresist is dissolved away exposing the layer of chrome. The chromium can be etched away using chromium etchant 
consisting of ceric ammonium nitrate and nitric acid. Finally, the glass is etched using a mixture of hydrofluoric acid, nitric acid, and water. The feature sizes can be controlled by the size of the features on the mask, etch time, and concentration of the $\mathrm{HF} / \mathrm{HNO}_{3}$ solution. The glass etching process is isotropic, etching equally in all directions, so it etches the depth of the channel as well as etching the sides. Therefore, channels in the glass will end up slightly larger than the channels on the mask and have a more trapezoidal shape than rectangular.

\subsubsection{Nanofluidic and Nanocapillary Membrane Fabrication}

Several research groups have started incorporating nanofluidic channels interfaced to microfluidic channels into their device designs to serve two main purposes thus far: 1) defined injection and fractionation ${ }^{69,70}$ and 2) sample concentration enhancement. ${ }^{43,44,48,51,52,67}$ Nanofluidic channels also have promise in separation techniques, single molecule detection, and fluidic diodes. Two approaches of coupling nanofluidic channels to microfluidic channels within devices have been executed; one of which connects microfluidic channels with nanochannels in the same piece of material as the microfluidic channels ${ }^{42,43,48,51,52}$ and the second uses membranes consisting of nanocapillaries to connect to the microfluidic channels. ${ }^{44}$

Nanochannels are typically characterized by their aspect ratio or the ratio between their height and width, which breaks them into three categories: low aspect ratio (width is on the micron scale and the depth is on the nanoscale), aspect ratio near one (both the width and depth are on the nanoscale), and high aspect ratio (width is on the nanoscale and the depth is on the micron scale). ${ }^{71}$ Many techniques of making nanochannels in devices have been devised including both bottom-up and top-down approaches. Bottom-up approaches arrange a material to create a nanosized feature and top-down approaches create nanosized features in a bulk material.

Thin film deposition and sacrificial layer techniques are both bottom-up methods to create nanochannels. Thin films can be either deposited or grown in a manner that results in nanochannels and larger features can be etched in the 
substrate to which the thin film is attached. ${ }^{72}$ Sacrificial layers can be spaced nanometers apart, a second layer can be cast onto the sacrificial layer, and finally the sacrificial layer is etched away leaving nanoscale channels. ${ }^{73}$

Top-down methods include photolithography, serial patterning techniques, and replication techniques. Photolithography is typically useful for creating nanochannels with low aspect ratios due to its inherent resolution limitations according to the Rayleigh equation shown in Equation 1.1 where $\mathrm{R}$ is the distance between the centers of evenly spaced patterns; $k_{1}$ is dependent on multiple factors including but not limited to the quality of the photomask, optics, and photoresist; $\lambda$ is the wavelength; and N.A. is the numerical aperture of the lens delivering light. ${ }^{71}$ Therefore, the wavelength of light (typically in the UV region) used to expose the photoresist becomes the limiting factor when attempting to pattern multiple nanochannels in parallel.

$$
R=\frac{k_{1} \lambda}{N . A}
$$

Scanning beam lithography (SBL) methods offer improved dimensions in comparison to photolithography. SBL techniques use energetic beams to bombard the substrate surface to create a pattern of nanochannels, which include electron beam lithography (EBL), ${ }^{74,75}$ focused ion beam (FIB), ${ }^{76-78}$ and scanning probe lithography (SPL). ${ }^{79,80} \mathrm{SBL}$ methods are typically compatible with both glass and polymer substrates. These methods can be time consuming, because a single nanochannel is created at a time instead of a parallel method that allows all the nanosized features to be made at once. Replication methods use a master to transfer a pattern onto a substrate by techniques such as nano imprint lithography (NIL), ${ }^{81}$ step-and-flash imprint lithography (SFIL), ${ }^{82}$ and nanotransfer printing (nTP). ${ }^{83}$ Often to use replication methods, the master or mold is made by using one of the serial patterning techniques but once the master is made the nanosized features can be made in parallel. ${ }^{71}$ The replication methods are more restrictive to the types of substrates that can be used. NIL is 
only compatible with thermoplastics and SFIL requires the use of a UV-curable liquid to create the nanochannel.

Another method to generate nanochannels is by exploiting the dielectric breakdown of materials. ${ }^{51,52}$ If an electric field is applied across two separate channels that it is stronger than the dielectric constant of the material, a channel will be formed connecting the two microchannels and its size is controlled by the strength of the electric field and the length of time the electric field is applied. However, this technique is only useful if a single nanochannel joining two larger microchannels is the desired result. These methods allow for the nanochannel to be in the same plane as the microfluidic channels and allow for simple imaging techniques to see on either side of the nanochannel effectively and have been reported in PDMS devices, glass devices, and glass/PDMS hybrid devices.

Several different types of membranes consisting of nanocapillaries can be implemented to serve as nanochannels within a device and will be referred to as nanocapillary membranes (NCM). Common materials used are alumina and polycarbonate, but both materials employ different methods to generate the nanocapillaries. Alumina membranes are formed through an anodization process in which a piece of aluminum is used as the anode. Oxygen is produced at the aluminum anode and is in close proximity to the surface of the aluminum and oxidizes it. The anodization process is typically carried out in a relatively strong acid solution in which the aluminum oxide is soluble allowing some of the aluminum oxide to be dissolved and continued exposure of the aluminum surface to the oxygen being produced. Typically, the anodization process is carried out twice. Following the first anodization, the aluminum oxide is removed and then the second anodization is performed to ensure a more uniform membrane. The anodization process results in nanocapillaries forming and being defined by aluminum oxide, but at the end of the nanocapillaries lies a barrier layer or a layer of aluminum oxide on top of the remaining aluminum. The nanocapillaries are then separated from the barrier layer and aluminum to result in an NCM with nanocapillaries that are open at either end. 
Polycarbonate membranes are a polymer, but the pores in the membrane are not a result of the polymerization process and can be more precisely controlled due to the fabrication technique. The technique to make track-etched membranes was first developed by P.B. Price and R.M. Walker in the 1960's as a method to more readily detect particles. ${ }^{84}$ They found that if a surface bombarded with ions (known at the time to produce tracks in the substances) was subsequently exposed to an appropriate etchant for the substrate, the tracks were enhanced. The production of nuclear track-etched membranes is based on this research in which the membrane is irradiated with charged particles in a nuclear reactor and followed by etching with sodium hydroxide. The nuclear track-etched membranes can be purchased with a range of different pore sizes as small as $10 \mathrm{~nm}$ and as large as $20 \mu \mathrm{m}$. The nanocapillaries of NCMs are smaller than most nanofluidic channels fabricated in devices to date, which offers advantages for the application of sample concentration enrichment. Buffer concentration becomes a critical parameter for sample concentration enrichment devices (this will be discussed later in more detail). The smaller nanocapillaries of NCMs allow for more concentrated buffers to be implemented such as $10 \mathrm{mM}$, which is more compatible with capillary electrophoresis experiments that will eventually be coupled to the analyte concentrator devices. In addition, implementing NCMs rather than fabricating nanochannels in the device allows for differential coating of the microfluidic channels and nanofluidic channels/capillaries for particular applications.

Nafion is also a membrane, but it does not contain straight regular nanopores. Nafion membranes are polymerized using two monomers, tetrafluoroethylene (Teflon) and a second monomer created from Teflon and sulfur trioxide. ${ }^{85}$ The resulting Nafion contains exposed sulfonate groups and can be extruded into the desired thickness and shape. The details of Nafion's structure have not been resolved, but in general, it is accepted that nanosized channels that have charged sulfonate groups on the surface make up the Nafion membrane. 


\subsection{Fluidic Transport}

Regardless of the fabrication technique used, transport of analyte in microfluidic devices is critical. Understanding the fluidic movement is important, because the characteristics of the fluid movement will affect the capabilities of the techniques being developed. An important characteristic of the fluid flow in microfluidic devices is its laminar nature meaning it is composed of multiple layers of solution moving in the same direction with minimal interaction with adjacent layers of solution. This type of movement results in little mixing and little random movement. The low mixing is a direct result of the dimensions of microfluidic channels and can be related through Equation 1.2 where $R_{e}$ is the Reynold's number, $d_{c}$ is the capillary diameter, $\rho$ is the solution density, $\langle v\rangle$ is the average fluid velocity, and $\eta$ is the viscosity of the solution.

$$
R_{e}=\frac{d_{c} \rho\langle v\rangle}{\eta}
$$

The Reynold's number is typically very low in microfluidic devices and not until the Reynold's number approaches 2000 does the regime of laminar begin transitioning into turbulent flow or flow with a much greater propensity to mix. ${ }^{86}$

Two typical methods of pumping bulk solution and manipulating samples are possible in microfluidic devices 1) hydrodynamic and 2) electrokinetic. Hydrodynamic flow can be generated by the application of a pressure differential or by differences in solution height between reservoirs. Electrokinetic flow is produced by applying an electric field across the microfluidic channels. Electrokinetic flow consists of two types of flow electrophoresis and EOF.

\subsubsection{Transport through Microfluidic Channels}

Hydrodynamic flow has a parabolic flow profile with the solution in the center traveling the fastest due to minimized frictional interaction with the wall of the channel as shown in Figure 1.2. ${ }^{86}$ 


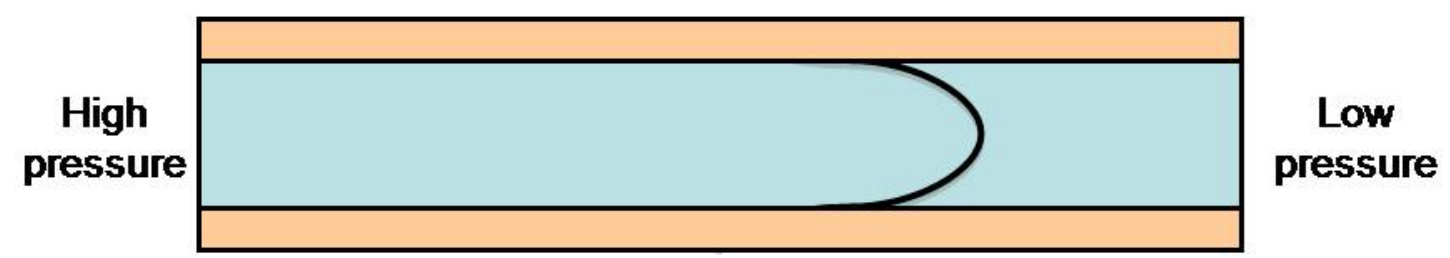

Figure 1.2: Schematic illustrating the parabolic flow profile of hydrodynamic flow in a microfluidic device.

Hydrodynamic flow can be useful for simple microfluidic devices. However, the non-uniform flow profile across the microchannel causes large band dispersion and damages separations. As more intersections are introduced onto the device, it becomes more difficult to control and steer the flow. The hydrodynamic flow will naturally follow the path of least resistance and often this is not the desired outcome. Therefore, electrokinetic flow becomes an appealing option with the ability to control the flow through voltages at multiple reservoirs electronically connected through the buffer in the microfluidic device.

Electrophoresis is the movement of an individual ion in response to an electric field through a medium as described by Equation 1.3 where $v$ is the velocity, $\mu_{\mathrm{ep}}$ is the electrophoretic mobility, and $E$ is the electric field.

$$
\begin{aligned}
& v=\mu_{e p} E \\
& \mu_{e p}=\frac{q}{f} E
\end{aligned}
$$

The electrophoretic mobility as shown in Equation 1.4 is dependent on the applied electric field, ion's charge (q), size, and shape and therefore the velocity depends on these characteristics as well. The size and shape are represented by $f$ the frictional force.

EOF is a bulk flow generated within capillaries that have a surface charge that is compensated with an electric double layer in solution. ${ }^{87}$ When a substrate such as glass is exposed to an aqueous solution, the groups on the surface of the glass can dissociate depending on solution $\mathrm{pH}$ causing a negative surface 
charge at $\mathrm{pH}$ values greater than the $\mathrm{pK}_{\mathrm{a}}$ of the surface groups. This surface charge, which is negative in the case of bare glass, must be balanced by positive charge in solution or the counter-ions. Therefore, a greater amount of positive charge than negative charge is found in solution near the capillary walls. The positive charge near the capillary walls is classified into two groups the stern layer and the diffuse layer as shown in Figure 1.3. The defining characteristic between the two layers for the following discussion is the movement of the ions. The stern layer is immobile while the ions of the diffuse layer are free to move.

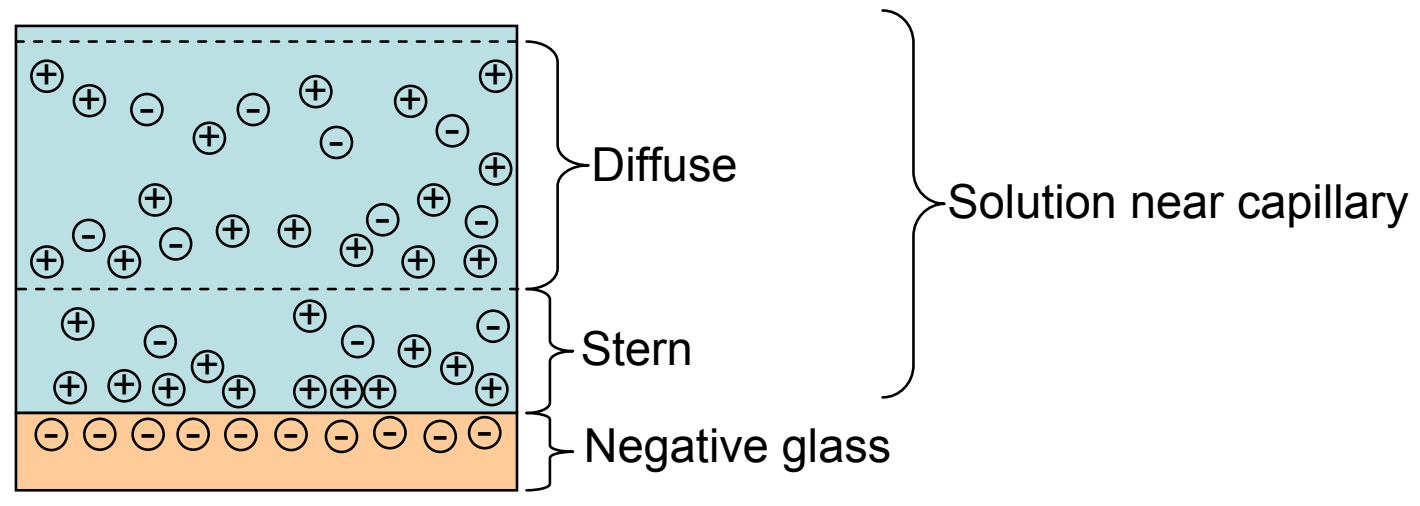

Figure 1.3: Schematic illustrating the double layer associated with a negative surface.

Once a voltage is applied, the positive charges move toward the cathode and due to their non-uniform distribution cause viscous coupling between the layers of solution and cause a bulk movement of solution to the cathode referred to as cathodic EOF. Therefore, in the presence of EOF it is possible for neutrals and anions to migrate to the cathode provided the magnitude of the EOF is greater than the mobility of the anions. If the surface charge of the substrate is altered to be positive, the direction of the EOF will be reversed and is frequently referred to as reverse EOF or anodic EOF. EOF, unlike hydrodynamic flow, provides a flat plug profile, which minimizes band broadening by providing a more uniform sample plug as shown in Figure 1.4. 


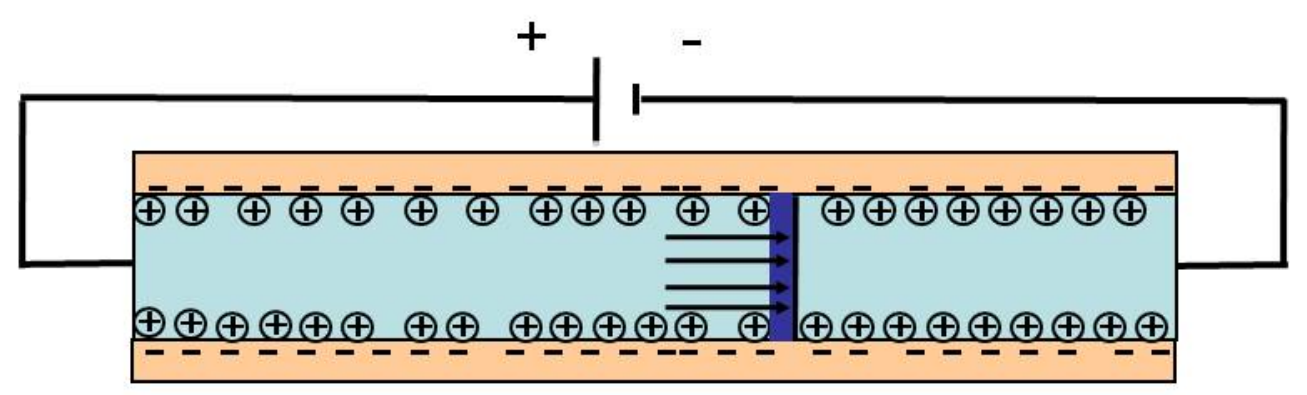

Figure 1.4: Schematic illustrating the flat plug profile of EOF and flow towards the cathode.

However, EOF can suffer similar steering issues as hydrodynamic flow, because EOF is a bulk flow it will generate pressure gradients within a device and will follow the path of least resistance once it reaches an intersection. Therefore, steering analyte through multiple intersections can become problematic once the pressure gradients are established. One method to direct the flow is to bias the reservoirs that terminate the intersecting channels such that the flow from these intersecting channels opposes the pressure. Typically, both electrophoresis and EOF are present at the same time, and the velocity of ions in solution are dependent on both as seen in Equation 1.5.

$$
v_{o b s}=v_{e p h}+v_{E O F}
$$

In Equation 1.5, $v_{\text {obs }}$ represents the observed velocity, $v_{\text {eph }}$ represents the velocity due to electrophoresis and $\mathrm{V}_{\mathrm{EOF}}$ represents the velocity due to EOF. Therefore, cations in solution will have a greater observed velocity in the presence of cathodic EOF in comparison to neutrals and anions. Anions will have the slowest velocity due to the opposite direction of their electrophoretic velocity in comparison to cathodic EOF.

\subsubsection{Transport through Nanofluidic Channels/Capillaries}

Transport through nanofluidic channels or NCMs is related but different from transport through microfluidic channels. Hydrodynamic transport through nanofluidics is extraordinarily small or slow due to the relationship between solution flux and the radius of the channel established by the Hagen-Poiseuille 
equation as shown in Equation 1.6 where $Q$ is the solution flux, $\Delta p$ is the change in pressure, $r$ is the radius of the channel, $L$ is the length of the channel, and $\eta$ is the viscosity. ${ }^{86}$

$$
Q=\frac{\pi \Delta p r_{c}^{4}}{8 L \eta}
$$

The above equation indicates that as the radius decreases and all other variables remain constant the flux of solution will decrease as a function of $r^{4}$. To maintain the same flow rate with different radii, a much greater pressure difference must be established to compensate for the change in radius. For instance, to generate a flow rate of $10 \mathrm{~nL} / \mathrm{min}$ through a $10 \mathrm{~nm}$ channel only 6 microns in length would require a pressure difference of $5.9 \times 10^{13} \mathrm{psi}$ according to Equation 1.6. The necessary pressure gradient required for hydrodynamic flow through nanosized channels is difficult to produce. Therefore, nanochannels rely on electrokinetic flow to transport solution.

Nanochannels have the same two categories of electrokinetic flow as microchannels, electrophoretic flow and EOF. However, the nanoscale influences the response to an electric field depending on solution concentration and nanochannel size. A double layer forms at the solid-liquid interface in the nanochannels as described in the section about transport through microfluidic devices. If the nanochannels are small enough and solution concentration is low enough as described by the Guoy-Chapman theory ${ }^{88}$ of the double layer as shown in Equation 1.7, the double layer can be on the same size as the nanochannel itself, a phenomena called double layer overlap as shown in Figure 1.5. ${ }^{89}$ The curve within the pore represents the potential profile and as it reaches the base of the pore the potential is zero. Therefore, at high ionic strength the counter-ion concentration reaches that of bulk while low ionic strength does not reach the bulk concentration of counter-ions as shown in Figure 1.5. In Equation 1.7, $\mathrm{C}$ is the molar concentration and $\mathrm{z}$ is the charge of the ions in solution. Therefore, nanochannels with smaller radii permit more concentrated buffers to be used while still producing double layer overlap. 


$$
\kappa=3.29 \times 10^{7} z C^{1 / 2}
$$

High Ionic Strength or Wide Channel

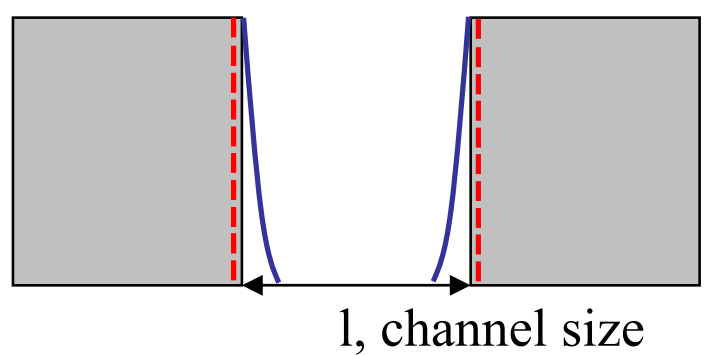

Low Ionic Strength or Narrow Channel

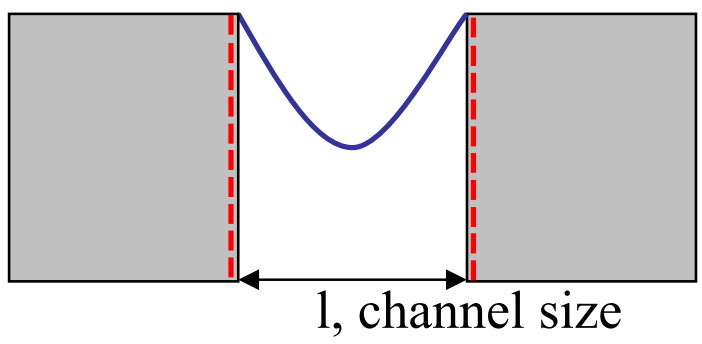

Figure 1.5: Schematic illustrating double layer overlap in negatively charged nanochannels or nanocapillaries at varying buffer concentrations. The blue line within the nanochannel represents the potential profile within the nanochannel.

Double layer overlap means that the double layer from one wall extends into the double layer formed from the opposite wall as shown in Figure 1.5, which indicates that the charge from the walls is not compensated for by solution. Therefore, the critical parameter in determining whether double layer overlap exists is the ratio of the double layer thickness to the nanochannel height. It should also be noted that the end of the double layer is defined as $1 / \mathrm{e}$, which is an arbitrary definition and the solution concentration is not equal to bulk at the defined end. In the case of high ionic strength buffer, enough cations are present to mask the surface charge of the nanochannels and a buffer concentration comparable to bulk is reached. If the channel is larger, this will change the ratio of the double layer thickness to the nanochannel height andlead to reduced double layer overlap. As the ionic strength of the buffer is decreased, less cations are present in solution and the double layer becomes larger extending further into the nanochannel from either side. Also, the nanochannel size can be reduced to increase the ratio between the double layer thickness and the nanochannel height while still remaining at relatively high buffer concentrations. Whether the double layer overlap is created by reducing the buffer concentration or the size of the nanochannel, ions in solution experience a net negative charge 
from the surface of the nanochannel that is typically compensated in larger microchannels. As a direct result of double layer overlap, nanocapillaries become ion permselective meaning that they preferentially transport either cations or anions. ${ }^{90}$ If the surface of the nanocapillary is negative, it is said to be cation permselective or transports cations at a greater rate than anions. If the surface of the nanocapillary is positive, it said to be anion permselective. From the ion permselectivity of nanochannels, an interesting phenomenon, concentratraion polarization, results when an electric field is applied across the nanochannel.

Concentration polarization (CP) ${ }^{87}$ occurs when an electric field is applied through a permselective material, such as a nanochannel and the cations are transported through the nanocapillary more readily than the anions as a result of double layer overlap, which has even been observed with larger bipolar membranes. ${ }^{91-93} \mathrm{CP}$ begins as cations are depleted from one side of the nanocapillary and because electroneutrality must be maintained, anions cannot remain in this region causing a zone depleted of ions to form on the anodic side of the nanocapillary. Likewise, an excess of cations accumulates on the opposite side of the membrane and anions pair with the cations to maintain electroneutrality. This process of $\mathrm{CP}$ results in an ion enriched zone forming on the cathodic side of the nanochannel and an ion depleted zone forming on the anodic side of the nanochannel (assuming a negatively charged surface in the nanocapillary) as shown in Figure 1.6. 


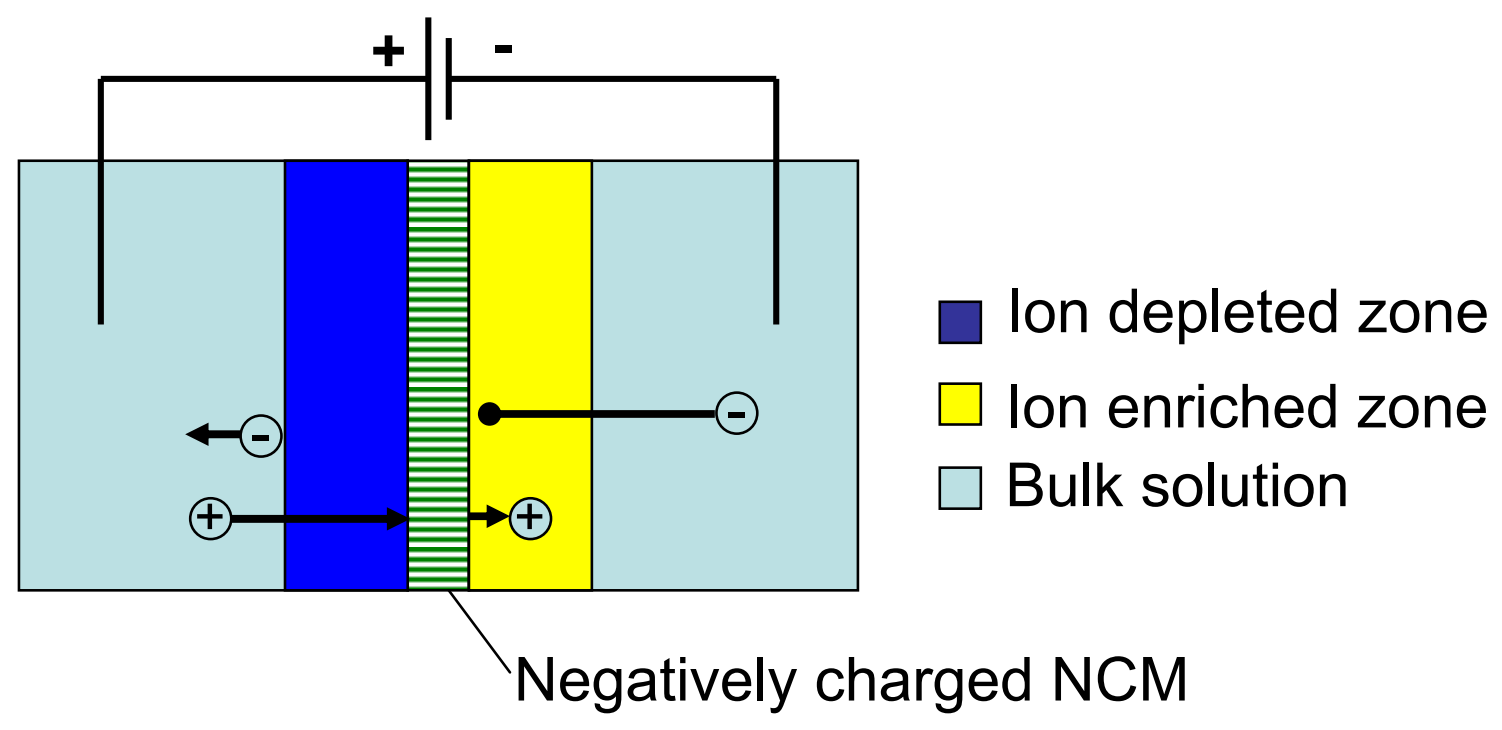

Figure 1.6: Schematic of concentration polarization.

\subsection{Nanofluidic/Microfluidic Interfaces}

When ion permselective nanochannels are interfaced with microchannels and an electric field is applied, CP develops. However, the microfluidic channel of the NMI interface bears unique characteristics namely low Reynold's numbers. ${ }^{86}$ The low Reynold's numbers allow the CP zones to remain relatively undisturbed. The sustained development of CP at NMIs has offered a phenomenon to utilize and develop for practical applications such as sample enrichment.

\subsection{Current Monitoring}

Because the mechanisms of transport through nanoscale systems are not fully understood, basic properties of the integrated device must be characterized. A simple but useful method is to monitor the current of a system when a voltage is applied. The current can be easily obtained by placing a resistor in series with the electrodes and measuring the voltage drop across the resistor. With the known resistance of the resistor and the measured voltage drop, the current can be calculated using Ohm's law. Monitoring the current profile may help explain or at least indicate a reason for observed behavior. For instance, it would be 
possible to observe current rectification where unequal amounts of current are passed at voltages of equal magnitude but opposite signs.

\subsection{Detection Methods}

Several different detection methods have been incorporated into

microfluidic devices including ultraviolet-visible absorbance, ${ }^{94-100}$ electrochemical, ${ }^{101-116}$ mass spectrometry, ${ }^{117-135}$ and fluorescence. ${ }^{14,51,136-144}$ Of these techniques fluorescence microscopy is the only method used within this work, because it is capable of imaging the chip which is critical and therefore the discussion will be limited to fluorescence microscopy.

Fluorescence microscopy can be used to detect sample at a single point or image the device within the field of view. Single point detection offers better limits of detection, but only provides information about one point on the device. If a charge-coupled device (CCD) is used as the detector, fluorescence microscopy is capable of providing an image of the device and makes it the most useful for developing new components for multicomponent devices. The imaging capability of fluorescence microscopy provides information about movement throughout the entire field of view instead of at a single point. Developing a component on a chip with only single point detection is difficult with the limited data about the overall movement of sample through the device. If the device is malfunctioning prior to the detector, the sample may not ever reach the detector and this is the only piece of data available. There is not any data to help indicate why the sample never reached the detector. Therefore, for the development of an on-chip microfluidic analyte concentrator an epifluorescence microscope system with a CCD detector has proven to be an indispensible tool.

\subsubsection{Epifluorescence microscopy}

Epifluorescence means that the excitation and emission light both pass through the same objective. In general, an epifluorescence microscope system is equipped with an excitation source, optics, and a detector as shown in Figure 1.7. Lasers or broadband sources may be incorporated into the system for 
excitation. Lasers offer higher intensity light than broadband sources, but at a single wavelength. The ability to supply a single wavelength of light can be both an advantage and disadvantage depending on the application. Lasers offer better limits of detection because of higher intensity light in comparison with broadband sources, which leads to greater fluorescence signal. However, all analytes must absorb the same wavelength of light. Therefore, a laser excitation source is less versatile because only a limited amount of analytes can be studied. A broadband source is less expensive and offers multiple wavelengths of light that can be isolated using bandpass filters. The ability to isolate multiple wavelengths of light allows analytes with different excitation and emission maxima to be used. Once the excitation light passes through the bandpass filter, the light interacts with a dichroic mirror. The dichroic mirror has the unique capability of reflecting particular wavelengths while simultaneously allowing others to pass through the dichroic mirror. Therefore, the excitation wavelength is reflected and reaches the sample or microfluidic device. The fluorescence emitted from the analyte within the microfluidic device can then be passed through the dichroic mirror. Lastly, an emission filter is used to reduce any scattered excitation or other light prior to detection. The detector is typically either a photomultiplier tube (PMT) or CCD camera. A PMT offers better limits of detection and faster data collection speeds, but only detects at the focal point. A CCD has inferior limits of detection in comparison with a PMT, but in return produces an image with $x-y$ coordinates. Therefore, multiple points of interest can be selected for detection. In addition, methods such as binning have been developed to improve the limits of detection of a CCD but not without sacrificing another aspect, resolution. Binning allows multiple pixels to be added together prior to being readout. The readout or amplification of the signal is the greatest source of noise in a CCD. ${ }^{145}$ Therefore, adding four pixels together, in a $2 \times 2$ bin, results in only one readout process instead of four. 


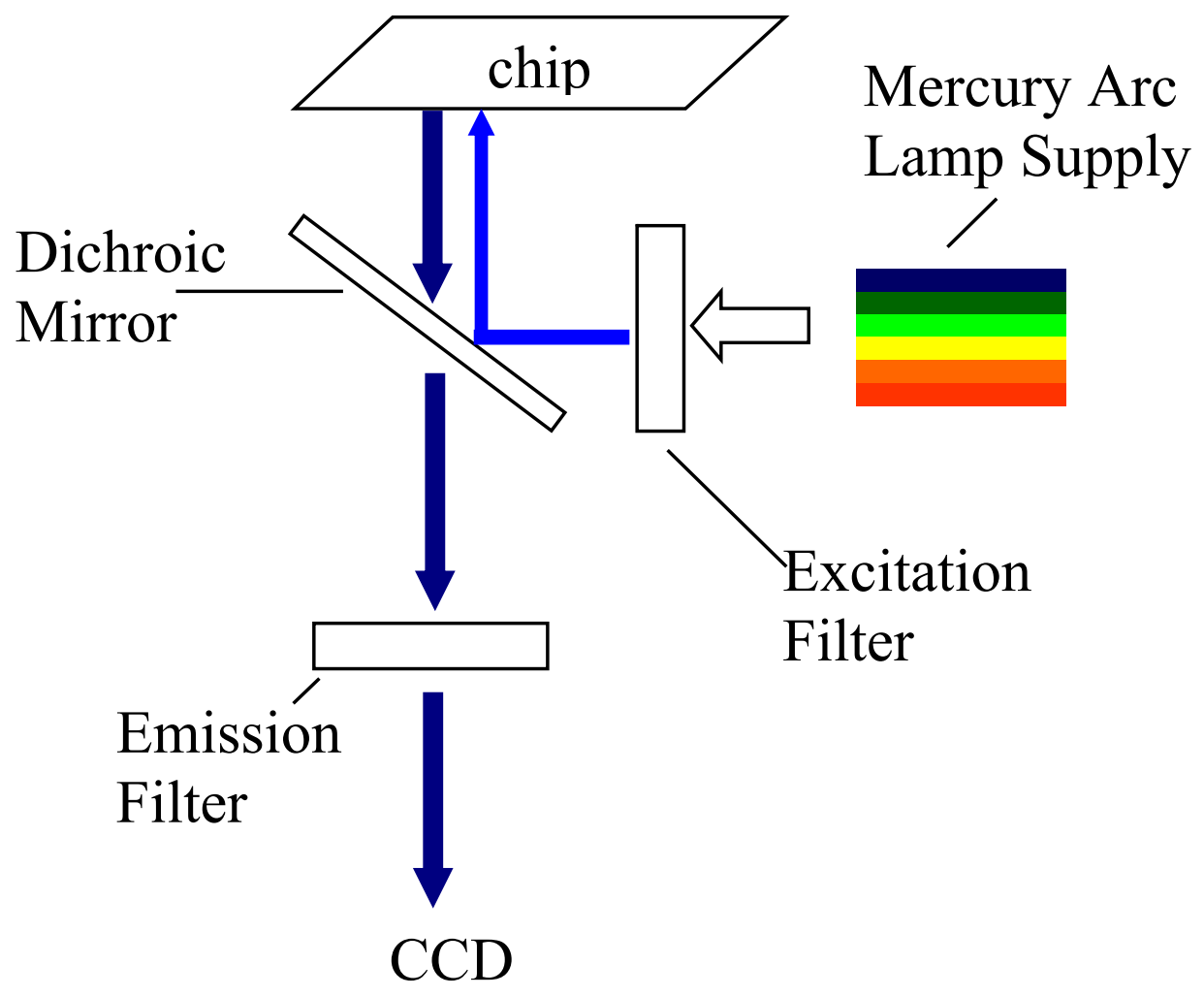

Camera

Figure 1.7: Schematic of an Epifluorescence system

While several methods of detection have been adapted for use with microfluidic devices, all must address both the benefits and obstacle of small sample consumption. In one respect the small sample consumption is a great advantage for the analysis of precious proteomic samples, but the obvious result of small sample consumption is the low amount of sample present for detection. Therefore, a method of ensuring the sample is concentrated enough to be detected is required within the microfluidic device.

\subsection{Proteomic Sample Analyses with Microfluidic Platforms}

The overall goal for proteomic microfluidic platforms is to create a miniature laboratory housed on a single platform that will not require direct handling of the sample once it is introduced. Therefore, an example of an integrated device for proteomics will allow for an HPLC separation to be carried out first. Prior to a CE separation, an array of concentrators would allow for 
enrichment of each band from the HPLC separation. Part of the concentrators could have trypsin incorporated with them causing the protein to be digested into peptides and resulting in both bottom-up and top-down proteomic data. The array of concentrators would be followed by CE separations to separate the peptides and proteins and finally electrospray ionization mass spectrometry for sequencing of the peptides and proteins. The entire device must also be coated to minimize protein adsorption to the surface of the device. The description of this integrated device is not the only possible combination of techniques for proteomics, but offers an idea of the complexity required to create a miniature laboratory for proteomics analysis. While there is still yet to be a report of a completely integrated and automated proteomic microfluidic device, much research has been performed concerning the individual components of an integrated microfluidic device. Each component of the miniature laboratory must be developed individually and then the components can be integrated with one another. This approach allows for studying the performance of each component as parameters are changed without the other steps influencing the component of interest. Many different research groups have contributed to the development of the different components including two-dimensional separations, ${ }^{146-148}$ protein digestion, ${ }^{149-151}$ concentration techniques, ${ }^{10-20,26-66}$ and MS interfaces. ${ }^{152-156}$ The concentrators are a critical component of these comprehensive proteomic sample analyzers, which need proper development of their own to ensure their robust function within such a complex system. 


\subsection{References}

(1) Liebler, D. C. Introduction to Proteomics: Tools for the New Biology; Humana Press Inc.: Totowa, NJ, 2002.

(2) Garrett, R. H. G., C.M. Biochemistry, Second ed.; Saunders College Publishing: Orlando, FL, 1999.

(3) Gygi, S. P.; Rochon, Y.; Franza, B. R.; Aebersold, R. Correlation between protein and mRNA abundance in yeast, Mol Cell Biol 1999, 19, 17201730.

(4) Wu, L.; Han, D. K. Overcoming the dynamic range problem in mass spectrometry-based shotgun proteomics, Expert review of proteomics 2006, 3, 611-619.

(5) Anderson, N. L.; Anderson, N. G. The human plasma proteome. History, character, and diagnostic prospects, Mol. Cell. Proteomics 2002, 1, 845867.

(6) Ghaemmaghami, S.; Huh, W.-K.; Bower, K.; Howson, R. W.; Belle, A.; Dephoure, N.; O'Shea, E. K.; Weissman, J. S. Global analysis of protein expression in yeast, Nature 2003, 425, 737-741.

(7) Hamdan, M.; Righetti, P. G. Assessment of Protein Expression by Means of 2-D Gel Electrophoresis with and without Mass Spectrometry, Mass Spectrometry Reviews 2003, 22, 272-284.

(8) Washburn, M. P.; Wolters, D.; Yates, J. R. Large-scale analysis of the yeast proteome by multidimensional protein identification technology, Nat Biotechnol 2001, 19, 242-247.

(9) Wolters, D. A.; Washburn, M. P.; Yates, J. R., III An automated multidimensional protein identification technology for shotgun proteomics, Anal. Chem. 2001, 73, 5683-5690.

(10) Yu, C.; Davey, M. H.; Svec, F.; Frechet, J. M. J. Monolithic porous polymer for on-chip solid-phase extraction and preconcentration prepared by photoinitiated in situ polymerization within a microfluidic device Anal Chem 2001, 73, 5088-5096.

(11) Oleschuk, R. D.; Shultz-Lockyear, L. L.; Ning, Y.; Harrison, D. J. Trapping of bead-based reagents within microfluidic systems. On-chip solid-phase extraction and electrochromatography Anal Chem 2000, 72, 585-590.

(12) Wen, J.; Guillo, C.; Ferrance, J. P.; Landers, J. P. DNA Extraction Using a Tetramethyl Orthosilicate-Grafted Photopolymerized Monolithic Solid Phase, Anal Chem 2006, 78, 1673-1681.

(13) Jemere, A. B.; Oleschuk, R. D.; Ouchen, F.; Fajuyigbe, F.; Harrison, D. J. An integrated solid-phase extraction system for sub-picomolar detection, Electrophoresis 2002, 23, 3537-3544.

(14) Long, Z.; Shen, Z.; Wu, D.; Qin, J.; Lin, B. Integrated Multilayer Microfluidic Device with a Nanoporous Membrane interconnect for Online Coupling of Solid-Phase Extraction to Microchip Electrophorsis, Lab on a Chip 2007, 7, 1819-1824.

(15) Yao, B.; Yang, H.; Liang, Q.; Luo, G.; Wang, L.; Ren, K.; Gao, Y.; Wang, Y.; Qu, Y. High-Speed, Whole-Column Fluorescence Imaging Detection 
for Isoelectric Focusing on a Microchip Using an Organic Light Emitting Diode as Light Source, Anal. Chem. 2006, 78, 5845-5850.

(16) Dauriac, V.; Descroix, S.; Chen, Y.; Peltre, G.; Senechal, H. Isoelectric focusing in an ordered micropillar array, Electrophoresis 2008, 29, 29452952.

(17) Cui, H.; Horiuchi, K.; Dutta, P.; Ivory Cornelius, F. Isoelectric focusing in a poly(dimethylsiloxane) microfluidic chip, Anal Chem 2005, 77, 1303-1309.

(18) Li, C.; Yang, Y.; Craighead, H. G.; Lee, K. H. Isoelectric focusing in cyclic olefin copolymer microfluidic channels coated by polyacrylamide using a UV photografting method, Electrophoresis 2005, 26, 1800-1806.

(19) Herr, A. E.; Molho, J. I.; Drouvalakis, K. A.; Mikkelsen, J. C.; Utz, P. J.; Santiago, J. G.; Kenny, T. W. On-chip coupling of isoelectric focusing and free solution electrophoresis for multidimensional separations, Anal. Chem. 2003, 75, 1180-1187.

(20) Huang, T.; Pawliszyn, J. Microfabrication of a tapered channel for isoelectric focusing with thermally generated $\mathrm{pH}$ gradient, Electrophoresis 2002, 23, 3504-3510.

(21) Masar, M.; Zuborova, M.; Kaniansky, D.; Stanislawski, B. Determination of oxalate in beer by zone electrophoresis on a chip with conductivity detection, J. Sep. Sci. 2003, 26, 647-652.

(22) Chen, L.; Prest, J. E.; Fielden, P. R.; Goddard, N. J.; Manz, A.; Day, P. J. R. Miniaturised isotachophoresis analysis, Lab Chip 2006, 6, 474-487.

(23) Kohlheyer, D.; Eijkel, J. C. T.; van den Berg, A.; Schasfoort, R. B. M. Miniaturizing free-flow electrophoresis - a critical review, Electrophoresis 2008, 29, 977-993.

(24) Jung, B.; Bharadwaj, R.; Santiago, J. G. On-Chip Millionfold Sample Stacking Using Transient Isotachophoresis, Anal. Chem. 2006, 78, 23192327.

(25) Xu, Z.; Nishine, T.; Arai, A.; Hirokawa, T. Performance of electrokinetic supercharging for high-sensitivity detection of DNA fragments in chip gel electrophoresis, Electrophoresis 2004, 25, 3875-3881.

(26) Sueyoshi, K.; Kitagawa, F.; Otsuka, K. Recent progress of online sample preconcentration techniques in microchip electrophoresis., J. Sep. Sci. 2008, 31, 2650-2666.

(27) Lichtenberg, J.; Verpoorte, E.; de Rooij, N. F. Sample preconcentration by field amplification stacking for microchip-based capillary electrophoresis., Electrophoresis 2001, 22, 258-271.

(28) Ring-Ling, C. Sample stacking revisited: a personal perspective, Electrophoresis 2003, 24, 486-497.

(29) Lin, H.; Shackman, J. G.; Ross, D. Finite sample effect in temperature gradient focusing, Lab Chip 2008, 8, 969-978.

(30) Kim, S. M.; Sommer, G. J.; Burns, M. A.; Hasselbrink, E. F. Low-Power Concentration and Separation Using Temperature Gradient Focusing via Joule Heating, Anal. Chem. 2006, 78, 8028-8035.

(31) Ross, D.; Locascio, L. E. Microfluidic temperature gradient focusing, Anal. Chem. 2002, 74, 2556-2564. 
(32) Hoebel, S. J.; Balss, K. M.; Jones, B. J.; Malliaris, C. D.; Munson, M. S.; Vreeland, W. N.; Ross, D. Scanning Temperature Gradient Focusing, Anal. Chem. 2006, 78, 7186-7190.

(33) Balss, K. M.; Vreeland, W. N.; Phinney, K. W.; Ross, D. Simultaneous concentration and separation of enantiomers with chiral temperature gradient focusing, Anal. Chem. 2004, 76, 7243-7249.

(34) Becker, M.; Mansouri, A.; Beilein, C.; Janasek, D. Temperature gradient focusing in miniaturized free-flow electrophoresis devices, Electrophoresis 2009, 30, 4206-4212.

(35) Kelly, R. T.; Woolley, A. T. Electric field gradient focusing, J. Sep. Sci. 2005, 28, 1985-1993.

(36) Hlushkou, D.; Perdue, R. K.; Dhopeshwarkar, R.; Crooks, R. M.; Tallarek, $U$. Electric field gradient focusing in microchannels with embedded bipolar electrode, Lab Chip 2009, 9, 1903-1913.

(37) Liu, J.; Sun, X.; Farnsworth, P. B.; Lee, M. L. Fabrication of Conductive Membrane in a Polymeric Electric Field Gradient Focusing Microdevice, Anal. Chem. 2006, 78, 4654-4662.

(38) Warnick, K. F.; Francom, S. J.; Humble, P. H.; Kelly, R. T.; Woolley, A. T.; Lee, M. L.; Tolley, H. D. Field gradient electrophoresis, Electrophoresis 2005, 26, 405-414.

(39) Kelly Ryan, T.; Li, Y.; Woolley Adam, T. Phase-changing sacrificial materials for interfacing microfluidics with ion-permeable membranes to create on-chip preconcentrators and electric field gradient focusing microchips, Anal Chem 2006, 78, 2565-2570.

(40) Greenlee, R. D.; Ivory, C. F. Protein Focusing in a Conductivity Gradient, Biotechnol. Prog. 1998, 14, 300-309.

(41) Petsev, D. N.; Lopez, G. P.; Ivory, C. F.; Sibbett, S. S. Microchannel protein separation by electric field gradient focusing, Lab Chip 2005, 5, 587-597.

(42) Pu, Q.; Yun, J.; Temkin, H.; Liu, S. Ion-Enrichment and Ion-Depletion Effect of Nanochannel Structures, Nano Lett 2004, 4, 1099-1103. Wang, Y.-C.; Stevens, A. L.; Han, J. Million-fold Preconcentration of Proteins and Peptides by Nanofluidic Filter, Anal Chem 2005, 77, 42934299.

(44) Zhang, Y.; Timperman, A. T. Integration of nanocapillary arrays into microfluidic devices for use as analyte concentrators, Analyst (Cambridge, United Kingdom) 2003, 128, 537-542.

(45) Khandurina, J.; Jacobson, S. C.; Waters, L. C.; Foote, R. S.; Ramsey, J. M. Microfabricated Porous Membrane Structure for Sample Concentration and Electrophoretic Analysis, Anal Chem 1999, 71, 1815-1819.

(46) Foote, R. S.; Khandurina, J.; Jacobson, S. C.; Ramsey, J. M. Preconcentration of proteins on microfluidic devices using porous silica membranes, AnalChem 2005, 77, 57-63.

(47) Dhopeshwarkar, R.; Sun, L.; Crooks, R. M. Electrokinetic concentration enrichment within a microfluidic device using a hydrogel microplug, Lab on a Chip 2005, 5, 1148-1154. 
(48) Dai, J.; Ito, T.; Sun, L.; Crooks, R. M. Electrokinetic trapping and concentration enrichment of DNA in a microfluidic channel, Journal of the American Chemical Society 2003, 125, 13026-13027.

(49) Song, S.; Singh, A. K.; Shepodd, T. J.; Kirby, B. J. Microchip dialysis of proteins using in situ photopatterned nanoporous polymer membranes, Anal Chem 2004, 76, 2367-2373.

(50) Kim, S. J.; Wang, Y.-C.; Lee, J. H.; Jang, H.; Han, J. Concentration polarization and nonlinear electrokinetic flow near a nanofluidic channel, Physical Review Letters 2007, 99, 044501/044501-044501/044504.

(51) Kim Sun, M.; Burns Mark, A.; Hasselbrink Ernest, F. Electrokinetic protein preconcentration using a simple glass/poly(dimethylsiloxane) microfluidic chip, Anal Chem 2006, 78, 4779-4785.

(52) Lee, J. H.; Chung, S.; Kim, S. J.; Han, J. Poly(dimethylsiloxane)-Based Protein Preconcentration Using a Nanogap Generated by Junction Gap Breakdown, Anal Chem 2007, 79, 6868-6873.

(53) Dhopeshwarkar, R.; Crooks Richard, M.; Hlushkou, D.; Tallarek, U. Transient effects on microchannel electrokinetic filtering with an ionpermselective membrane, Anal Chem 2008, 80, 1039-1048.

(54) Huang, K.-D.; Yang, R.-J. A nanochannel-based concentrator utilizing the concentration polarization effect, Electrophoresis 2008, 29, 4862-4870.

(55) Wang, S.-C.; Wei, H.-H.; Chen, H.-P.; Tsai, M.-H.; Yu, C.-C.; Chang, H.-C. Dynamic superconcentration at critical-point double-layer gates of conducting nanoporous granules due to asymmetric tangential fluxes, Biomicrofluidics 2008, 2, 014102.

(56) Stein, D.; Deurvorst, Z.; Heyden, F. H. J. v. d.; Koopmans, W. J. A.; Gabel, A.; Dekker, C. Electrokinetic Concentration of DNA Polymers in Nanofluidic Channels, Nano Lett 2010, DOI: 10.1021/nl902228p.

(57) Hoeman, K. W.; Lange, J. J.; Roman, G. T.; Higgins, D. A.; Culbertson, C. T. Electrokinetic trapping using titania nanoporous membranes fabricated using sol-gel chemistry on microfluidic devices, Electrophoresis 2009, 30, 3160-3167.

(58) Huang, K.-D.; Yang, R.-J. Formation of ionic depletion/enrichment zones in a hybrid micro-/nano-channel, Microfluid Nanofluid 2008, 5, 631-638.

(59) Wu, D.; Steckl, A. J. High speed nanofluidic protein accumulator, Lab on a Chip 2009, 9, 1890-1896.

(60) Yamamoto, S.; Hirakawa, S.; Suzuki, S. In Situ Fabrication of Ionic Polyacrylamide-Based Preconcentrator on a Simple Poly(methylmethacrylate) Microfluidic Chip for Capillary, Anal. Chem. 2008, 80, 8224-8230.

(61) Kovarik, M. L.; Jacobson, S. C. Integrated Nanopore/Microchannel Devices for ac Electrokinetic Trapping of Particles, Anal. Chem. 2008, 80, 657-664.

(62) Lee, J. H.; Song, Y.-A.; Han, J. Multiplexed proteomic sample preconcentration device using surface-patterned ion-selective membrane, Lab on a Chip 2008, 8, 596-601. 
(63) Wang, Y.-C.; Han, J. Pre-binding dynamic range and sensitivity enhancement for immuno-sensors using nanofluidic preconcentrator, Lab on a Chip 2008, 8, 392-394.

(64) Kim, S. J.; Han, J. Self-Sealed Vertical Polymeric Nanoporous-Junctions for High-Throughput Nanofluidic Applications, Anal. Chem. 2008, 80, 3507-3511.

(65) Zhou, K.; Kovarik, M. L.; Jacobson, S. C. Surface-Charge Induced Ion Depletion and Sample Stacking near Single Nanopores in Microfluidic Devices, J. Am. Chem. Soc. 2008, 130, 8614-8616.

(66) Holtzel, A.; Tallarek, U. Ionic conductance of nanopores in microscale analysis systems: where microfluidics meets nanofluidics, J Sep Sci 2007, 30, 1398-1419.

(67) Song, S.; Singh Anup, K.; Kirby Brian, J. Electrophoretic concentration of proteins at laser-patterned nanoporous membranes in microchips, Anal Chem 2004, 76, 4589-4592.

(68) Hlushkou, D.; Dhopeshwarkar, R.; Crooks Richard, M.; Tallarek, U. The influence of membrane ion-permselectivity on electrokinetic concentration enrichment in membrane-based preconcentration units, Lab on a chip 2008, 8, 1153-1162.

(69) Cannon, D. M., Jr.; Kuo, T.-C.; Bohn, P. W.; Sweedler, J. V. Nanocapillary Array Interconnects for Gated Analyte Injections and Electrophoretic Separations in Multilayer Microfluidic Architectures, Anal Chem 2003, 75, 2224-2230.

(70) Tulock, J. J.; Shannon, M. A.; Bohn, P. W.; Sweedler, J. V. Microfluidic Separation and Gateable Fraction Collection for Mass-Limited Samples, Anal Chem 2004, 76, 6419-6425.

(71) Abgrall, P.; Nguyen, N. T. Nanofluidic Devices and Their Applications, Anal. Chem. 2008, 80, 2326-2341.

(72) Schoch, R. B.; van Lintel, H.; Renaud, P. Effect of the surface charge on ion transport through nanoslits, Phys. Fluids 2005, 17, 100604/100601$100604 / 100605$.

(73) Gajar, S. A.; Geis, M. W. An ionic liquid-channel field-effect transistor, J. Electrochem. Soc. 1992, 139, 2833-2840.

(74) Lercel, M. J.; Craighead, H. G.; Parikh, A. N.; Seshadri, K.; Allara, D. L. Plasma etching with self-assembled monolayer masks for nanostructure fabrication, J. Vac. Sci. Technol., A 1996, 14, 1844-1849.

(75) Yasin, S.; Hasko, D. G.; Ahmed, H. Fabrication of $<5 \mathrm{~nm}$ width lines in poly(methyl methacrylate) resist using a water:isopropyl alcohol developer and ultrasonically-assisted development, Appl. Phys. Lett. 2001, 78, 27602762.

(76) Campbell, L. C.; Wilkinson, M. J.; Manz, A.; Camilleri, P.; Humphreys, C. J. Electrophoretic manipulation of single DNA molecules in nanofabricated capillaries, Lab Chip 2004, 4, 225-229.

(77) Mahabadi, K. A.; Rodriguez, I.; Haur, S. C.; van Kan, J. A.; Bettiol, A. A.; Watt, F. Fabrication of PMMA micro- and nanofluidic channels by proton 
beam writing: electrokinetic and morphological characterization, $\mathrm{J}$. Micromech. Microeng. 2006, 16, 1170-1180.

(78) Arscott, S.; Troadec, D. Electrospraying from nanofluidic capillary slot, Appl. Phys. Lett. 2005, 87, 134101/134101-134101/134103.

(79) Kramer, S.; Fuierer Ryan, R.; Gorman Christopher, B. Scanning probe lithography using self-assembled monolayers, Chem Rev 2003, 103, 4367-4418.

(80) Geissler, M.; Xia, Y. Patterning: Principles and some new developments, Adv. Mater. 2004, 16, 1249-1269.

(81) Chou, S. Y.; Krauss, P. R.; Renstrom, P. J. Imprint of sub-25 $\mathrm{nm}$ vias and trenches in polymers, Appl. Phys. Lett. 1995, 67, 3114-3116.

(82) Ruchhoeft, P.; Colburn, M.; Choi, B.; Nounu, H.; Johnson, S.; Bailey, T.; Darmle, S.; Stewart, M.; Ekerdt, J.; Sreenivasan, S. V.; Wolfe, J. C.; Willson, C. G. Patterning curved surfaces: Template generation by ion beam proximity lithography and relief transfer by step and flash imprint lithography, J. Vac. Sci. Technol., B 1999, 17, 2965-2969.

(83) Loo, Y.-L.; Willett, R. L.; Baldwin, K. W.; Rogers, J. A. Interfacial Chemistries for Nanoscale Transfer Printing, J. Am. Chem. Soc. 2002, 124, 7654-7655.

(84) Price, P. B.; Walker, R. M. Chemical etching of charged-particle tracks in solids, J. Appl. Phys. 1962, 33, 3407-3412.

(85) Grot, W. Discovery and development of Nafion perfluorinated membranes, Chem. Ind. (London) 1985, 647-649.

(86) Giddings, J. C. Unified Separation Science, 1991.

(87) Probstein, R. F. Physicochemical Hydrodynamics. An Introduction, 1989.

(88) Bard, A. J.; Faulkner, L. R. Electrochemical Methods, Fundamentals and Applications, 2nd ed.; John Wiley \& Sons, 2001.

(89) Rubinstein, I.; Zaltzman, B. In Surface Chemistry and Electrochemistry of Membranes; Sorensen, T. S., Ed., 1999; Vol. 79, pp 591-621.

(90) Plecis, A.; Schoch Reto, B.; Renaud, P. Ionic transport phenomena in nanofluidics: experimental and theoretical study of the exclusionenrichment effect on a chip, Nano Lett 2005, 5, 1147-1155.

(91) Lovrecek, B.; Despic, A.; Bockris, J. O. M. Electrolytic junctions with rectifying properties, Journal of Physical Chemistry 1959, 63, 750-751.

(92) Suendo, V.; Eto, R.; Tanioka, A. Ionic Rectification Properties of a Bipolar Interface Consisting of a Cationic Surfactant and Cation-Exchange Membrane, Journal of Colloid and Interface Science 2002, 250, 507-509.

(93) Hurwitz, H. D.; Dibiani, R. Experimental and theoretical investigations of steady and transient states in systems of ion exchange bipolar membranes, Journal of Membrane Science 2004, 228, 17-43.

(94) Gaspar, A.; Bacsi, I.; Garcia, E. F.; Braun, M.; Gomez, F. A. Application of external micro-spectrophotometric detection to improve sensitivity on microchips, Anal. Bioanal. Chem. 2009, 395, 473-478.

(95) Mogensen, K. B.; Petersen, N. J.; Hubner, J.; Kutter, J. P. In-plane UV absorbance detection in silicon-based electrophoresis devices using 
monolithically integrated optical waveguides, Micro Total Anal. Syst. 2001, Proc. 2001, 280-282.

(96) Salimi-Moosavi, H.; Jiang, Y.; Lester, L.; McKinnon, G.; Harrison, D. J. A multireflection cell for enhanced absorbance detection in microchip-based capillary electrophoresis devices, Electrophoresis 2000, 21, 1291-1299.

(97) Newman, C. I. D.; Giordano, B. C.; Copper, C. L.; Collins, G. E. Microchip micellar electrokinetic chromatography separation of alkaloids with UVabsorbance spectral detection, Electrophoresis 2008, 29, 803-810.

(98) Jindal, R.; Cramer, S. M. On-chip electrochromatography using sol-gel immobilized stationary phase with UV absorbance detection, $\mathrm{J}$. Chromatogr., A 2004, 1044, 277-285.

(99) Ma, B.; Zhou, X.; Wang, G.; Dai, Z.; Qin, J.; Lin, B. A hybrid microdevice with a thin PDMS membrane on the detection window for UV absorbance detection, Electrophoresis 2007, 28, 2474-2477.

(100) Yeung, E. S.; Pang, H.-M.; Han, F.; (Combisep, Inc., USA). Application: US

US, 2006, pp 20 pp.

(101) Buttgenbach, S.; R.Wilke A Capillary Electrophoresis Chip with Hydrodynamic Sample Injection for Measurements from a Continuous Sample Flow, Analytical Bioanalytical Chemistry 2005, 383, 733-737.

(102) Liu, Y.; Fanguy, J. C.; Bledsoe, J. M.; Henry, C. S. Dynamic Coating Using Polyelectrolyte Multilayers for Chemical Control of Electroosmotic Flow in Capillary Electrophoresis Microchips, Analytical Chemistry 2000 72, 59395944.

(103) Wang, J.; Chen, G.; Muck, A., Jr.; Collins, G. E. Electrophoretic microchip with dual-opposite injection for simultaneous measurements of anions and cations, Electrophoresis 2003, 24, 3728-3734.

(104) Castano-Alvarez, M.; Fernandez-Abedul, M. T.; Costa-Garcia, A.; Agirregabiria, M.; Fernandez, L. J.; Ruano-Lopez, J. M.; Barredo-Presa, B. Fabrication of SU-8 based microchip electrophoresis with integrated electrochemical detection for neurotransmitters, Talanta 2009, 80, 24-30.

(105) Fredrick, S. J.; Gross, E. M. Use of microelectrodes for electrochemiluminescent detection in microfluidic devices, Bioanalysis 2009, 1, 31-36.

(106) Dungchai, W.; Chailapakul, O.; Henry, C. S. Electrochemical Detection for Paper-Based Microfluidics, Analytical Chemistry 2009, 81, 5821-5826.

(107) Nugen, S. R.; Asiello, P. J.; Connelly, J. T.; Baeumner, A. J. PMMA biosensor for nucleic acids with integrated mixer and electrochemical detection, Biosens. Bioelectron. 2009, 24, 2428-2433.

(108) Mecker, L. C.; Martin, R. S. Integration of Microdialysis Sampling and Microchip Electrophoresis with Electrochemical Detection, Analytical Chemistry 2008, 80, 9257-9264.

(109) Lin, K.-W.; Huang, Y.-K.; Su, H.-L.; Hsieh, Y.-Z. In-channel simplified decoupler with renewable electrochemical detection for microchip capillary electrophoresis, Analytica Chimica Acta 2008, 619, 115-121. 
(110) Shiddiky, M. J. A.; Park, D.-S.; Shim, Y.-B. Detection of polymerase chain reaction fragments using a conducting polymer-modified screen-printed electrode in a microfluidic device, Electrophoresis 2005, 26, 4656-4663.

(111) Wang, J.; Pumera, M.; Chatrathi, M. P.; Rodriguez, A.; Spillman, S.; Martin, R. S.; Lunte, S. M. Thick-film electrochemical detectors for poly(dimethylsiloxane)-based microchip capillary electrophoresis, Electroanalysis 2002, 14, 1251-1255.

(112) Gonzalez, C. F.; Cropek, D. M.; Henry, C. S. Photopatternable Carbon Electrodes for Chip-Based Electrochemical Detection, Electroanalysis 2009, 21, 2171-2174.

(113) Garcia, C. D.; Henry, C. S. Coupling capillary electrophoresis and Pulsed electrochemical detection, Electroanalysis 2005, 17, 1125-1131.

(114) Garcia, C. D.; Henry, C. S. Comparison of pulsed electrochemical detection modes coupled with microchip capillary electrophoresis, Electroanalysis 2005, 17, 223-230.

(115) Garcia, C. D.; Henry, C. S. Direct detection of renal function markers using microchip CE with pulsed electrochemical detection, Analyst 2004, 129, 579-584.

(116) Garcia, C. D.; Henry, C. S. Enhanced determination of glucose by microchip electrophoresis with pulsed amperometric detection, Analytica Chimica Acta 2004, 508, 1-9.

(117) Ramsey, R. S.; Ramsey, J. M. Generating Electrospray from Microchip Devices Using Electroosmotic Pumping, Anal Chem 1997, 69, 1174-1178.

(118) Xue, Q.; Foret, F.; Dunayevskiy, Y. M.; Zavracky, P. M.; McGruer, N. E.; Karger, B. L. Multichannel Microchip Electrospray Mass Spectrometry, Anal Chem 1997, 69, 426-430.

(119) Lazar, I. M.; Li, L.; Yang, Y.; Karger, B. L. Microfluidic Device for Capillary Electrochromatography-Mass Spectrometry, Electrophoresis 2003, 24, 3655-3662.

(120) Zhang, B.; Liu, H.; Karger, B. L.; Foret, F. Microfabricated Devices for Capillary Electrophoresis-Electrospray Mass Spectrometry, Anal Chem 1999, 71, 3258-3264.

(121) Lazar, I. M.; Karger, B. L. Multiple Open-Channel Electroosmotic Pumping System for Microfluidic Sample Handling, Anal Chem 2002, 74, 62596268.

(122) Wang, C.; Oleshuk, R. D.; Ouchen, F.; Li, J.; Thibault, P.; Harrison, D. J. Integration of Immobilized Trypsin Bead Beds for Protein Digestion within a Microfluidic Chip Incorporating Capillary Electrophoresis Separations and an Electrospray Mass Spectrometry Interface, Rapid Communications in Mass Spectrometry 2000, 14, 1377-1383.

(123) Li, J.; Wang, C.; Kelly, J. F.; Harrison, D. J.; Thibault, P. Rapid and Sensitive Separation of Trace Level Protein Digests Using Microfabricated Devices Coupled to a Quadrupole-Time-of-Flight Mass Spectrometer, Electrophoresis 2000, 21, 198-210. 
(124) Pinto, D. M.; Ning, Y.; Figeys, D. An Enhanced Microfluidic Chip Coupled to an Electrospray Qstar Mass Spectrometer for Protein Identification, Electrophoresis 2000, 21, 181-190.

(125) Chan, J. H.; Timperman, A. T.; Aebersold, R. Microfabricated Polymer Devices for Automated Sample Delivery of Peptides for Analysis by Electrospray Ionization Tandem Mass Spectrometry, Anal Chem 1999, 71, 4437-4444.

(126) Figeys, D.; Gygi, S. P.; McKinnon, G.; Aebersold, R. An Integrated Microfluidics-Tandem Mass Spectrometry System for Automated Protein Analysis, Anal Chem 1998, 70, 3728-3734.

(127) Lazar, I. M.; Ramsey, R. S.; Jacobson, S. C.; Foote, R. S.; Ramsey, J. M. Novel Microfabricated Device for Electrokinetically Induced Pressure Flow and Electrospray Ionization Mass Spectrometry, Journal of Chromatography A 2000, 892, 195-201.

(128) Figeys, D.; Aebersold, R. Nanoflow Solvent Gradient Delivery from a Microfabricated Device for Protein Identification by Electrospray Ionization Mass Spectrometry, Anal Chem 1998, 70, 3721-3727.

(129) Lazar, I. M.; Ramsey, R. S.; Sundberg, S.; Ramsey, J. M. SubattomoleSensitivity Microchip Nanoelectrospray Source with Time-of-Flight Mass Spectrometry Detection, Anal Chem 1999, 71, 3627-3631.

(130) Figeys, D.; Ning, Y.; Aebersold, R. A Microfabricated Device for Rapid Protein Identification by Microelectrospray Ion Trap Mass Spectrometry, Anal Chem 1997, 69, 3153-3160.

(131) Tachibana, Y.; Otsuka, K.; Terabe, S.; Arai, A.; Suzuki, K.; Nakamura, S. Robust and Simple Interface for Microchip Electrophoresis-Mass Spectrometry, Journal of Chromatography A 2003, 1011, 181-192.

(132) Akashi, S.; Suzuki, K.; Arai, A.; Yamada, N.; Suzuki, E.-I.; Hirayama, K.; Nakamura, S.; Nishimura, Y. Top-Down Analysis of Basic Proteins by Microchip Capillary Electrophoresis Mass Spectrometry, Rapid Communications in Mass Spectrometry 2006, 20, 1932-1938.

(133) Li, J.; LeRiche, T.; Tremblay, T.-L.; Wang, C.; Bonneil, E.; Harrison, D. J.; Thibault, P. Application of Microfluidic Devices to Proteomics Research, Molecular \& Cellular Proteomics 2002, 1, 157-168.

(134) Li, J.; Kelly, J. F.; Chernushevich, I.; Harrison, D. J.; Thibault, P. Separation and Identification of Peptides from Gel-Isolated Membrane Proteins Using a Microfabricated Device for Combined Capillary Electrophoresis/Nanoelectrospray Mass Spectrometry, Anal Chem 2000, 72, 599-609.

(135) Li, J.; Thibault, P.; Bings, N. H.; Skinner, C. D.; Wang, C.; Colyer, C.; Harrison, D. J. Integration of Microfabricated Devices to Capillary Electrophoresis-Electrospray Mass Spectrometry Using a Low Dead Volume Connection: Application to Rapid Analyses of Proteolytic Digests, Anal Chem 1999, 71, 3036-3045.

(136) Mohamad Mohamadi; Noritada Kaji; Manabu Tokeshi; Baba, Y. Online Preconcentration by Transient Isotachophoresis in Linear Polymer on a Poly(methyl methacrylate) Microchip for Separation of Human Serum 
Albumin Immunoassay Mixtures, Analytical Chemistry 2007, 79, 36673672.

(137) Proczek, G.; Augustin, V.; Descroix, S.; Hennion, M.-C. Integrated microdevice for preconcentration and separation of a wide variety of compounds by electrochromatography, Electrophoresis 2009, 30, 515524.

(138) Long, Z.; Liu, D.; Ye, N.; Qin, J.; Lin, B. Integration of nanoporous membranes for sample filtration/preconcentration in microchip electrophoresis, Electrophoresis 2006, 27, 4927-4934.

(139) Hatch, A. V.; Herr, A. E.; Throckmorton, D. J.; Brennan, J. S.; Singh, A. K. Integrated Preconcentration SDS-PAGE of Proteins in Microchips Using Photopatterned Cross-Linked Polyacrylamide Gels, Analytical Chemistry 2006, 78, 4976-4984.

(140) Kim, B. Y.; Yang, J.; Gong, M.; Flachsbart, B. R.; Shannon, M. A.; Bohn, P. W.; Sweedler, J. V. Multidimensional Separation of Chiral Amino Acid Mixtures in a Multilayered Three-Dimensional Hybrid Microfluidic/Nanofluidic Device, Analytical Chemistry 2009, 81, 2715-2722.

(141) Beyor, N.; Yi, L.; Seo, T. S.; Mathies, R. A. Integrated Capture, Concentration, Polymerase Chain Reaction, and Capillary Electrophoretic Analysis of Pathogens on a Chip, Analytical Chemistry 2009, 81, 35233528.

(142) Said Attiya; Abebaw B. Jemere; Thompson Tang; Glen Fitzpatrick; Kurt Seiler; Nghia Chiem; Harrison, D. J. Design of an Interface to Allow Microfluidic Electrphoresis Chips to Drink from the Fire Hose of the External Environment, Electrophoresis 2001, 22, 318-327.

(143) Shu-Hui Chen; Yi-Hung Lin; Lan-Yu Wang; Chun-Che Lin; Gwo-Bin Lee Flow-Through Sampling for Electrophoresis-Based Microchips and Their Applications for Protein Analysis, Analytical Chemistry 2002, 74, 51465153.

(144) Yang, X.; Zhang, X.; Li, A.; Zhu, S.; Huang, Y. Comprehensive twodimensional separations based on capillary high-performance liquid chromatography and microchip electrophoresis, Electrophoresis 2003, 24, 14511457.

(145) Colarusso, P.; Spring Kenneth, R. Imaging at low light levels with cooled and intensified charge-coupled device cameras, Methods Enzymol 2003, 360, 383-394.

(146) Kim, B. Y.; Yang, J.; Gong, M.; Flachsbart, B. R.; Shannon, M. A.; Bohn, P. W.; Sweedler, J. V. Multidimensional Separation of Chiral Amino Acid Mixtures in a Multilayered Three-Dimensional Hybrid Microfluidic/Nanofluidic Device, Anal Chem 2009, 81, 2715-2722.

(147) Cong, Y.; Zhang, L.; Tao, D.; Liang, Y.; Zhang, W.; Zhang, Y. Miniaturized two-dimensional capillary electrophoresis on a microchip for analysis of the tryptic digest of proteins, Journal of Separation Science 2008, 31, 588594. 
(148) Ramsey, J. D.; Jacobson, S. C.; Culbertson, C. T.; Ramsey, J. M. TwoDimensional Separations of Protein Digests on Microfluidic Devices, Anal Chem 2003, 75, 3758-3764.

(149) Li, J.; LeRiche, T.; Tremblay, T.-L.; Wang, C.; Bonneil, E.; Harrison, D. J.; Thibault, P. Application of Microfluidic Devices to Proteomics Research I, Molecular \& Cellular Proteomics 2002, 1, 157-168.

(150) Wang, C.; Oleschuk, R.; Ouchen, F.; Li, J.; Thibault, P.; Harrison, D. J. Integration of immobilized trypsin bead beds for protein digestion within a microfluidic chip incorporating capillary electrophoresis separations and an electrospray mass spectrometry interface, Rapid Communications in Mass Spectrometry 2000, 14, 1377-1383.

(151) Dodge, A.; Brunet, E.; Chen, S.; Goulpeau, J.; Labas, V.; Vinh, J.; Tabeling, P. PDMS-based microfluidics for proteomic analysis, Analyst 2006, 131, 1122-1128.

(152) Ramsey, R. S.; Ramsey, J. M. Generating Electrospray from Microchip Devices Using Electroosmotic Pumping, Anal Chem 1997, 69, 1174-1178.

(153) Xue, Q.; Foret, F.; Dunayevskiy, Y. M.; Zavracky, P. M.; McGruer, N. E.; Karger, B. L. Multichannel Microchip Electrospray Mass Spectrometry, Anal Chem 1997, 69, 426-430.

(154) Lazar, I. M.; Ramsey, R. S.; Sundberg, S.; Ramsey, J. M. SubattomoleSensitivity Microchip Nanoelectrospray Source with Time-of-Flight Mass Spectrometry Detection, Anal Chem 1999, 71, 3627-3631.

(155) Akashi, S.; Suzuki, K.; Arai, A.; Yamada, N.; Suzuki, E.-I.; Hirayama, K.; Nakamura, S.; Nishimura, Y. Top-Down Analysis of Basic Proteins by Microchip Capillary Electrophoresis Mass Spectrometry, Rapid Communications in Mass Spectrometry 2006, 20, 1932-1938.

(156) Tachibana, Y.; Otsuka, K.; Terabe, S.; Arai, A.; Suzuki, K.; Nakamura, S. Robust and Simple Interface for Microchip Electrophoresis-Mass Spectrometry, Journal of Chromatography A 2003, 1011, 181-192. 


\title{
Chapter 2
}

\section{Ionic Current Rectification at a Nanofluidic/Microfluidic Interface with an Asymmetric Microfluidic System}

This chapter was previously published by Scott A. Miller, Kathleen C. Kelly (Reschke), and Aaron T. Timperman and appears here with minor changes, (Lab on a Chip, 2008, 8, 1729-1732).

\begin{abstract}
A nanofluidic-microfluidic interface is reported that rectifies ionic current using uncoated symmetric nanocapillaries. Previously, ionic current rectification has been achieved by other groups with nanochannels with differential coatings and in nanopores that are conical in shape. This simple device uses nanocapillary membranes (NCMs) with uncoated symmetric channels to connect a microfluidic channel and a larger solution reservoir. The conductivity of the solution in the microchannel appears to be critical in the formation of the low "off" state current and the high "on" state current. It is hypothesized that the "off" state current is low due to the formation of an ion depletion zone in the microchannel while the higher "on" state currents are produced by a zone of enhanced ionic concentration in the microchannel.
\end{abstract}




\subsection{Introduction}

The fields of clinical diagnostics, proteomics, genomics, and metabolomics require analytical tools with improved detection limits and separations compared with the tools currently available. Microfluidic devices and integrated systems have the ability to meet the requirements of such research areas, and to integrate and automate many sample handling steps on a microfluidic chip. Integrated systems composed of nanofluidic-microfluidic interfaces (NMIs) have proven to be capable of two main functions, increasing analyte concentration ${ }^{1-7}$ and acting as molecular gates or valves. ${ }^{8,9}$ With NMls providing a means to concentrate, inject, or fractionate sample in a multiplexed microfluidic system, it will be possible to carry out multiple sample preparation steps and complex separations in a high-throughput format. Therefore, NMls can be used to directly address two of the challenges faced with microfluidic devices, improving detection limits by increasing concentration, and improving integration of multiple processes through the use of molecular gates. NMls exploit the unique mass transport properties of nanocapillaries and nanochannels, which arise from the double layer overlap that occurs in the nanocapillaries that are on the same order as the size of the double layer. ${ }^{10}$ Double layer overlap causes the nanocapillaries or nanochannels to be permselective, diminishing the transport of co-ions. When ions are driven through permselective materials electrokinetically, zones of enhanced and depleted ion concentration develop.

Ion current rectification is a direct result of differential ion transport, and therefore ionic current rectifiers may find applications, such as controlling the concentration of ionic solutes in solution. ${ }^{11}$ Ionic current rectifiers are similar to diodes, and currently there is interest in the combination of electrical engineering design principles to the design of microfluidic systems from components, such as a fluidic diode. ${ }^{12}$ It is anticipated that ionic current rectifiers will be applied in similar applications.

Other groups have investigated ionic-current rectification with conicallyshaped pores and differentially coated nanochannels. The conical nanopores are unique in the sense that the pores span the nanofluidic-microfluidic 
continuum along their length. The pore diameters are typically on the order of a micron at the wide end (the "base") and ten nanometers at the narrow end (the "tip"). The negatively-charged conical-pore membranes only support current flow in one direction - when anion transport is in the base-to-tip direction. Siwy and Martin showed that this phenomenon is surface charge dependent by coating an asymmetric NCM with positive moieties and observing a reversal of current directionality. ${ }^{13}$ Umehara et al published similar results for conical quartz nanopipettes. ${ }^{14}$ Siwy recently reviewed a number of proposed models interpreting ion current rectification in asymmetric conical nanopores. ${ }^{15}$ Two models of note attribute rectification to zones of enhanced or depleted electrolyte in the ion-selective nanopore for the "on" and "off" states respectively. ${ }^{16-18}$

More recently, ionic current rectification was reported with nanofluidic channels that were differentially coated by giving one end of the nanochannels a positive surface charge and the other end of the nanochannels a neutral charge. ${ }^{11}$ This approach can be considered to be a nanofluidic corollary of the ionic current rectification observed with larger bipolar membranes. ${ }^{19-21}$ Theoretical work predicted that the current behavior of differentially charged nanochannels would be similar to a semiconductor diode. ${ }^{22}$

Plecis et al investigated transport through a nanochannel slit both experimentally and theoretically. ${ }^{23}$ They found that double layer overlap causes the nanochannel to be ion permselective and that electrostatic forces dominate the observed behavior. Pu et al also observed ion depletion and enrichment zones with a nanochannel/microchannel interface. ${ }^{24}$ Flux models based on permselectivity of nanochannels were used to explain the ion depletion/enrichment effect. Wang et al, reported the formation of an ion depletion zone in front of a nanochannel, and used it to provide $10^{6}$ to $10^{8}$ enhancement in analyte concentration. ${ }^{7}$

Herein, ionic current rectification is reported using uncoated nanochannels that are symmetric in geometry and surface charge, and are interfaced with an asymmetric fluidic system. In this system, the geometry of the larger fluidic system is asymmetric as the NCM connects a microfluidic channel with a larger 
reservoir. With this relatively simple fabrication technique that does not require differential coating or asymmetric nanocapillaries, rectification factors as high as 120 have been achieved at $\pm 800 \mathrm{~V}$. It is hypothesized that the ionic permselectivity of the nanochannels, which is a consequence of double layer overlap, plays a pivotal role in the observed current rectification with the asymmetric fluidic system.

\subsection{Experimental}

The devices used in this work, were fabricated of $5 \times 10 \mathrm{~cm}$ soda-lime glass from Telic (Valencia, CA) coated with Cr metal and AZ1500 photoresist using conventional photolithography and wet chemical etching processes as reported elsewhere. ${ }^{25}$ The microfluidic chips contained a 2-cm long microchannel with $210 \mu \mathrm{m} \times 12 \mu \mathrm{m}$ cross-sections.

For the device design shown in Figure 2.1, 150- $\mu \mathrm{m}$ diameter access holes were drilled at each end of the microchannel with a microdrilling station equipped with a Model 7000 drill press from Servo Products Company (Pasadena, CA), and $150-\mu \mathrm{m}$ drill bits from Kyocera Tycom Corporation (Irvine, CA).

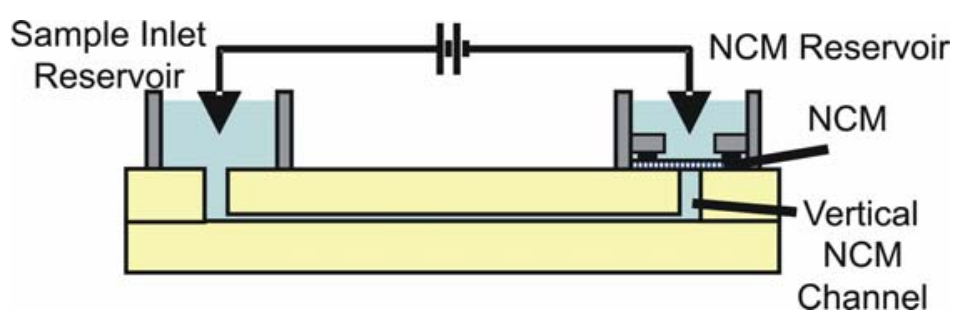

Figure 2.1: Schematic of NMI in which the NCM connects a microfluidic channel and macroscopic NCM reservoir. The design shown in (A) has a microfluidic channel with a $200-\mu \mathrm{m} x$ 13- $\mu \mathrm{m}$ horizontal portion and a vertical portion with a 150- $\mu \mathrm{m}$ ID and 1-mm length. The high voltage power supply (HVPS) supplies the potential, and the polarity of the potential is referenced to the voltage applied to the NCM reservoir.

Following bonding of the wafers, an Upchurch Nanoport reservoir was attached at one access hole and a custom-built, black Delrin reservoir housing 
was attached at the outlet where the NCM is placed. The housing was designed to accept a Teflon reservoir with a size 001 O-ring that seals the NCM over the mouth of the access hole or the end of the microchannel.

Prior to each experiment, the microchannel was rinsed with $0.1 \mathrm{M}$ sodium hydroxide, deionized water obtained from a Barnstead International Nanopure Infinity (Dubuque, IA) system, and $10 \mathrm{mM}$ sodium phosphate (Fisher Scientific) electrolyte solution adjusted to $\mathrm{pH} 7$ with $0.1 \mathrm{M}$ sodium hydroxide unless otherwise stated. The nanofluidic element was placed into the housing and a retaining ring was used to compress the nanofluidic element between the O-ring and the surface of the microfluidic chip. A track-etched polycarbonate(PC) NCM from Osmonics with $10 \mathrm{~nm}$ pores, a thickness of $6 \mu \mathrm{m}$, and porosity of $6 \times 10^{8}$ pores $/ \mathrm{cm}^{2}$; or a DuPont Nafion-112 membrane from Alpha Aesar was used as the nanofluidic element. The reservoirs and microchannel were filled with the 10 $\mathrm{mM}$ phosphate buffer.

A program written in Labwindows 8.0 from National Instruments controlled the output of a TREK high voltage power supply (610E) and recorded the current data. Typically, $-800 \mathrm{~V}$ was applied and allowed to reach a steady current followed by a switch to $+800 \mathrm{~V}$ which was again allowed to reach a steady current. The voltage was switched back to $-800 \mathrm{~V}$ and the program was started. The program applied $-800,-600,-500,-400,-300,-200,-150,-100,-50,-25,-10$, $0,10,25,50,100,150,200,300,400,500,600$, and finally 800 volts. Similar currents, exhibiting the same trend in current rectification, were obtained regardless of whether the voltages were scanned down from $+/-800 \mathrm{~V}$ or up from zero. A $10 \mathrm{~K} \Omega$ resistor was placed in series in the sample inlet reservoir grounding line, and the voltage drop across this resistor was measured and converted to current using Ohm's law. The average current for each voltage yielded the I-V curves shown in Figures 2.2 and 2.3. For the I-V plot generated in Figure 2.2, eight replicates were performed. For the I-V plot generated in Figure 2.3, three replicate runs were collected. For the Nafion experiments, the current stability at higher voltages was increased by connecting larger electrolyte reservoirs that contained $10 \mathrm{~mL}$ of solution to the on-chip reservoirs that 
contained $100 \mu \mathrm{L}$ of solution using a 5 -cm long $500 \mu \mathrm{m}$ ID PEEK connecting tube. When the $10 \mathrm{~mL}$ external reservoirs were used, the electrodes were placed in these reservoirs to minimize changes in the solution caused by electrolytic processes. Care was taken to keep all solution reservoirs at the same level.

\subsection{Results and Discussion}

The asymmetry of the fluidic system arises from having a microfluidic channel on one side of the NCM and a macroscopic fluidic reservoir on the other side of the NCM as shown in Figure 2.1. The passage of current through the system is determined by the resistance of its components. The equivalent circuit consists of a number of resistors connected in series, making the total resistance equal to the sum of the resistors. Therefore, if any one resistance is very large it will dominate the resistance of the overall system. The resistance of each region of the fluidic system is determined by its cross-sectional area (A), conductivity $(\sigma)$, and length $(\mathrm{L})$ as shown by equation $1 .^{26}$

$$
R=L / A \sigma
$$

In this system, with background electrolyte or buffer initially homogenous, changes in the conductivity are caused by changes in ionic concentration. To increase the conductivity of the system above levels observed with no NCM, the resistance of the current limiting region(s) must be decreased.

The current rectification of the system is apparent in the I-V curve shown in Figure 2.2. The current is greatly reduced when a negative potential is applied to the NCM reservoir, which is referred to as the "off" state. The reduction in current is substantial as the "off" state is about $1 / 15$ of the Ohmic current that is observed when the NCM is not present. At voltages greater than $\sim 200 \mathrm{~V}$, a slight increase in current above the no NCM case is observed. At $\pm 800 \mathrm{~V} \mathrm{a}$ rectification factor greater than $17 x$ is achieved. 


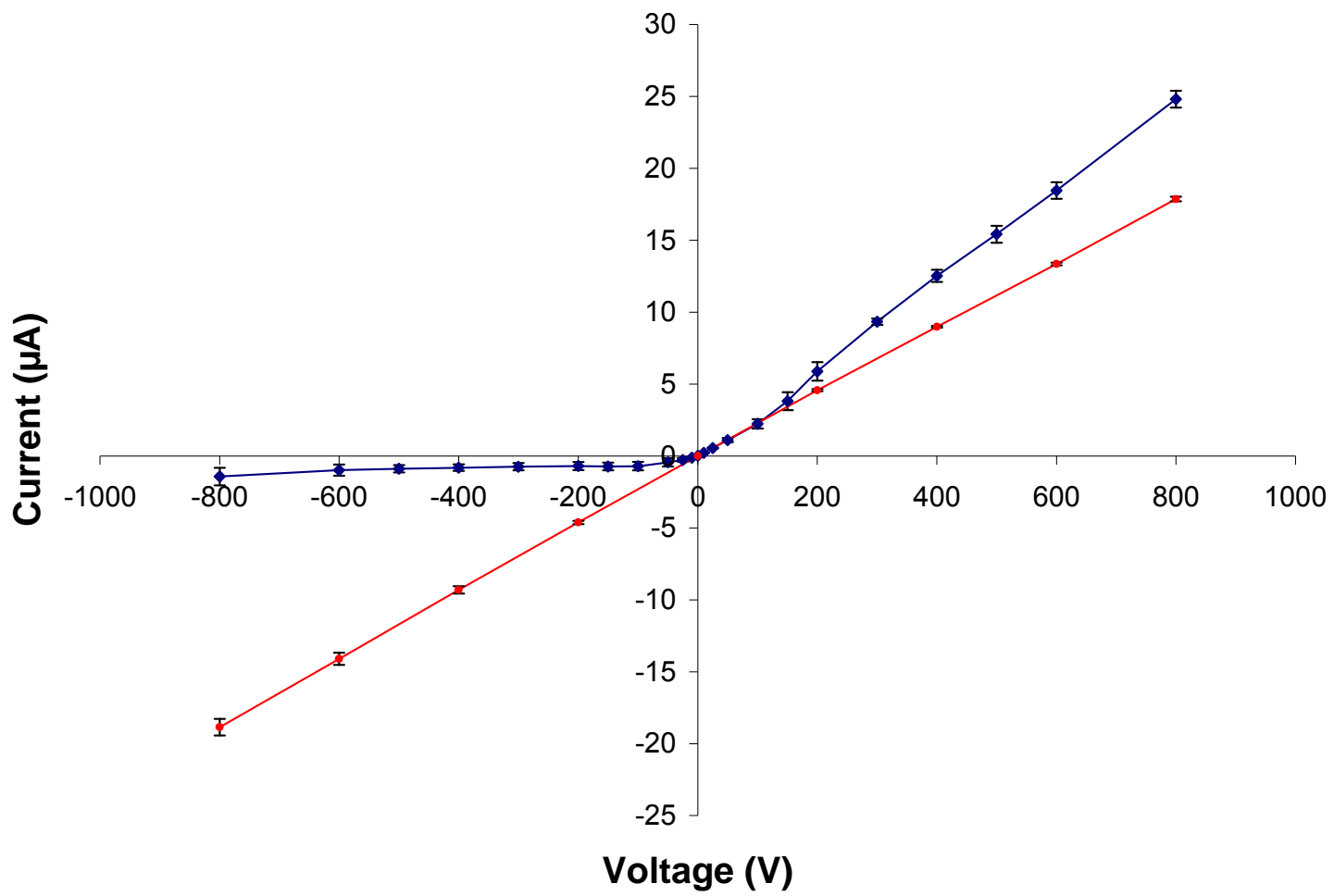

Figure 2.2: I-V curve in which the average currents are plotted as a function of potential applied across the microchannel interface $(\diamond)$ with a NCM and $(\bullet)$ without a NCM. Current rectification is observed with the lower currents obtained at negative potentials, compared with the high currents obtained at positive potentials. Negative voltages correspond to a negative voltage applied to the NCM reservoir.

\subsection{1 "Off" State}

The "off" state is characterized by a current that is much less than the Ohmic currents observed in the control in which the NCM is absent. In this system, the "off" state is observed when a negative potential is applied to the NCM reservoir. The suppressed transport of co-ions (anions in this case) through the NCM causes the formation of a ion depletion in the microfluidic channel, adjacent to the $\mathrm{NCM} .^{7,24}$ The depletion zone is stabilized by the small dimensions of the microchannel that yield a low Reynold's number. The formation of zones of enhanced and depleted ion concentration on opposite sides of a permselective material in electokinetically driven systems is consistent with accepted theory.$^{10,27}$ Zones of ion depletion and enhancement have been 
observed on opposite sides of a permselective nanochannel by Pu et al. In a similar study Wang et al observed the formation of a zone of ion depletion at a NMI. ${ }^{7,}{ }^{24}$ Although the cross-sectional area of the NCM is reduced by its low porosity, it does not appear to have a large influence on the total resistance of the system, mostly likely due to its short length $(\sim 6 \mu \mathrm{m})$. The resistance of the NCM is believed to be largely unaffected by the polarity of the applied potential. Regardless of the polarity of the applied potential, the interior of the nanocapillaries is believed to be dominated by the negative surface charge and the compensating diffuse layer.

\subsection{2 "On" State}

In the "on" state, it is proposed that a zone of ion enrichment forms in the microfluidic channel adjacent to the NCM a zone of ion enrichment forms in the macroscopic reservoir adjacent to the NCM. The higher "on" state currents indicate that the ion depletion zone does not reduce the overall current to "off" state current levels when it forms in the macroscopic reservoir. The reduced role of the ion depletion zone in determining the overall current when it forms in the macroscopic reservoir is thought to be a direct result of the greater crosssectional area of the macroscopic reservoir compared to the microchannel. This increase in cross-sectional area decreases the length of the ion depletion zone and allows for 3-dimensional diffusion to reduce its magnitude. Although a current limiting region observed with bipolar membranes is not observed here ${ }^{28}$ convective processes, such as electroconvection, may be important at higher voltages.

At positive potentials greater than $\sim 200-V$, the observed "on" state currents are slightly greater than the Ohmic currents recorded with the NCM. These above "Ohmic" currents are proposed to result from the formation of a zone of ion enrichment in the microchannel. As in the "off" state, due to its small cross-sectional area, the microchannel dominates the resistance of the overall system. At voltages between 0 and $\sim 200-\mathrm{V}$, the increase in current caused by the initial formation of enhanced ion concentration in the microchannel may be 
canceled out by the formation of the zone of ion depletion in the NCM reservoir that appears to be stable in this low voltage range.

\subsubsection{Nafion Membrane}

In an effort to better understand the role of cation-permselectivity on ion current rectification, the 10-nm PC NCM was replaced with a Nafion membrane in Figure 2.3.

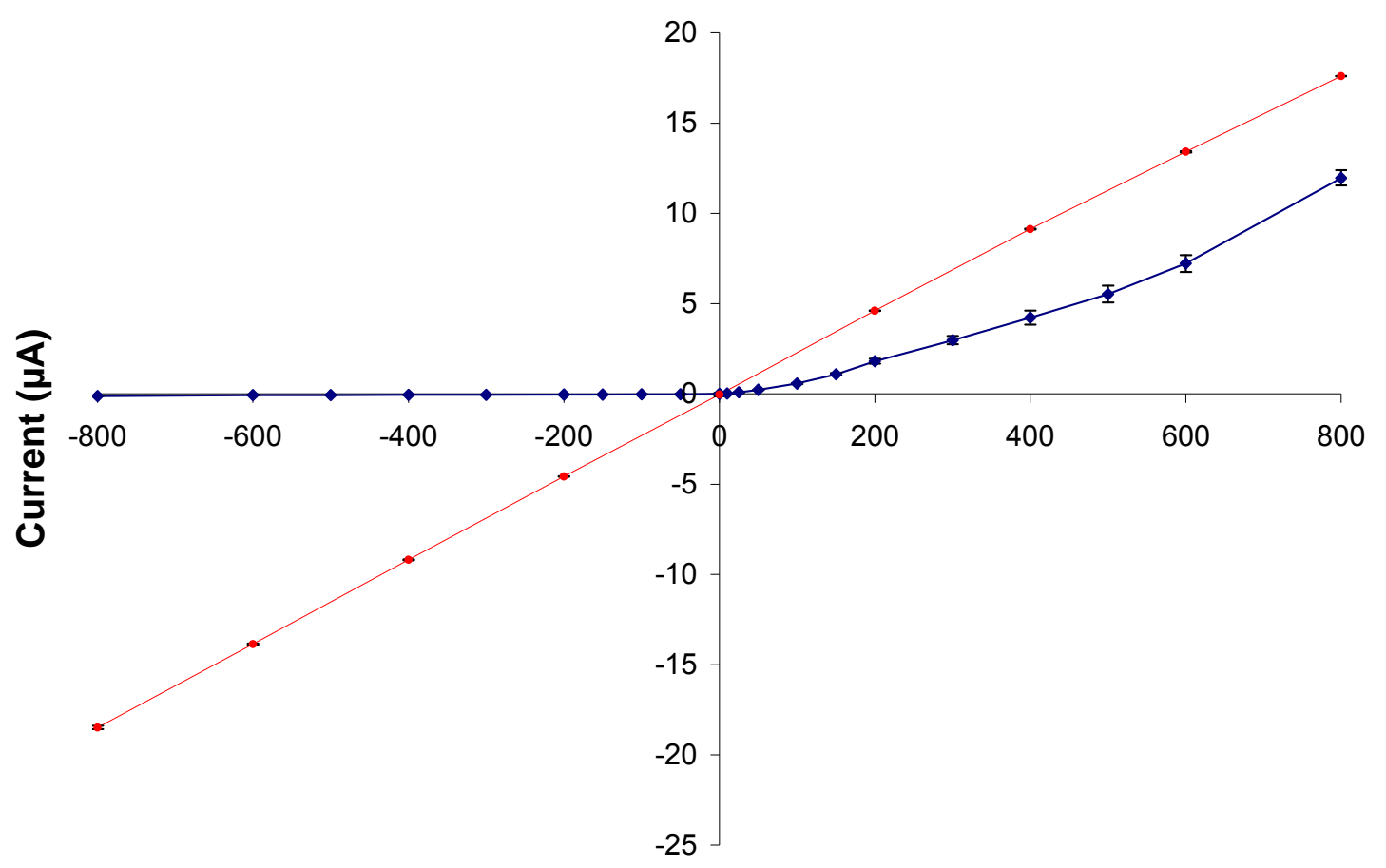

Voltage (V)

Figure 2.3: I-V curve in which the average current was plotted as a function of potential applied across the microchannel side-interfaced $(\diamond)$ with a Nafion-117 proton exchange membrane and $(\bullet)$ without a membrane. Nafion has greater cation permselectivity and consequently much lower currents are observed at negative applied potentials than are observed with the 10-nm PC NCMs as shown in Figure 2.2.

Nafion membranes have a much greater anionic surface charge, i.e. are more cation-permselective. It should be noted that the Nafion membrane is informative, but is not the ideal control, because it also has a smaller nominal 
nanocapillary diameter and does not have the relatively straight and symmetric pores characteristic of an NCM. With the Nafion membrane, the "off" state current is reduced with the current only reaching $-0.11 \mu \mathrm{A}$ at $-800 \mathrm{~V}$, a small fraction of the current observed in the no-membrane case. The currents for the "on" state are slightly below the current observed for the no-membrane case. Overall, in the Nafion-NMI, the "on" current was 99-fold greater than the "off" current compared to 17 -fold for the NCM-NMI.

\subsection{Conclusion}

The current rectification behavior between an NCM-NMI and a Nafion-NMI is compared. The Nafion-NMI has greater rectification factors, because the Nafion is more cation permselective than the NCM. The Nafion allows even fewer anions to pass causing even lower "off" state currents. The rectification of both devices is attributed to the asymmetric fluidic design about either the NCM or the Nafion membrane. The "off" state current is believed to be caused by the formation of a stable ion depletion zone in the microchannel while during the "on" state a zone of ion enrichment forms in the microchannel allowing for more current to pass through the device. Therefore, the changes in the conductivity of the solution within the microchannel appears to be a critical parameter in creating the low "off" state current and the high "on" state current. 


\subsection{References}

(1) Zhang, Y.; Timperman, A. T. Integration of nanocapillary arrays into microfluidic devices for use as analyte concentrators, Analyst (Cambridge, United Kingdom) 2003, 128, 537-542.

(2) Dai, J.; Ito, T.; Sun, L.; Crooks, R. M. Electrokinetic trapping and concentration enrichment of DNA in a microfluidic channel, Journal of the American Chemical Society 2003, 125, 13026-13027.

(3) Dhopeshwarkar, R.; Sun, L.; Crooks, R. M. Electrokinetic concentration enrichment within a microfluidic device using a hydrogel microplug, Lab on a Chip 2005, 5, 1148-1154.

(4) Foote, R. S.; Khandurina, J.; Jacobson, S. C.; Ramsey, J. M. Preconcentration of proteins on microfluidic devices using porous silica membranes, AnalChem 2005, 77, 57-63.

(5) Khandurina, J.; Jacobson, S. C.; Waters, L. C.; Foote, R. S.; Ramsey, J. M. Microfabricated Porous Membrane Structure for Sample Concentration and Electrophoretic Analysis, Anal Chem 1999, 71, 1815-1819.

(6) Song, S.; Singh, A. K.; Shepodd, T. J.; Kirby, B. J. Microchip dialysis of proteins using in situ photopatterned nanoporous polymer membranes, Anal Chem 2004, 76, 2367-2373.

(7) Wang, Y.-C.; Stevens, A. L.; Han, J. Million-fold Preconcentration of Proteins and Peptides by Nanofluidic Filter, Anal Chem 2005, 77, 42934299.

(8) Cannon, D. M., Jr.; Kuo, T.-C.; Bohn, P. W.; Sweedler, J. V. Nanocapillary Array Interconnects for Gated Analyte Injections and Electrophoretic Separations in Multilayer Microfluidic Architectures, Anal Chem 2003, 75, 2224-2230.

(9) Gatimu, E. N.; King, T. L.; Sweedler, J. V.; Bohn, P. W. Three-dimensional integrated microfluidic architectures enabled through electrically switchable nanocapillary array membranes, Biomicrofluidics 2007, 1.

(10) Probstein, R. F. Physicochemical Hydrodynamics. An Introduction, 1989.

(11) Karnik, R.; Duan, C.; Castelino, K.; Daiguji, H.; Majumdar, A. Rectification of ionic current in a nanofluidic diode, Nano Lett 2007, 7, 547-551.

(12) Adams, M. L.; Johnston, M. L.; Scherer, A.; Quake, S. R. Polydimethylsiloxane based micro-fluidic diode, Journal of Micromechanics and Microengineering 2005, 15, 1517-1521.

(13) Siwy, Z.; Heins, E.; Harrell, C. C.; Kohli, P.; Martin, C. R. ConicalNanotube Ion-Current Rectifiers: The Role of Surface Charge, Journal of the American Chemical Society 2004, 126, 10850-10851.

(14) Umehara, S.; Pourmand, N.; Webb Chris, D.; Davis Ronald, W.; Yasuda, K.; Karhanek, M. Current rectification with poly-I-lysine-coated quartz nanopipettes, Nano Lett 2006, 6, 2486-2492. 
(15) Siwy, Z. S. Ion-current rectification in nanopores and nanotubes with broken symmetry, Advanced Functional Materials 2006, 16, 735-746.

(16) Cervera, J.; Schiedt, B.; Ramirez, P. A Poisson/Nernst-Planck model for ionic transport through synthetic conical nanopores, Europhysics Letters 2005, 71, 35-41.

(17) Woermann, D. Electrochemical transport properties of a cone-shaped nanopore: high and low electrical conductivity states depending on the sign of an applied electrical potential difference, Physical Chemistry Chemical Physics 2003, 5, 1853-1858.

(18) Woermann, D. Electrochemical transport properties of a cone-shaped nanopore: revisited, Physical Chemistry Chemical Physics 2004, 6, 31303132.

(19) Lovrecek, B.; Despic, A.; Bockris, J. O. M. Electrolytic junctions with rectifying properties, Journal of Physical Chemistry 1959, 63, 750-751.

(20) Suendo, V.; Eto, R.; Tanioka, A. Ionic Rectification Properties of a Bipolar Interface Consisting of a Cationic Surfactant and Cation-Exchange Membrane, Journal of Colloid and Interface Science 2002, 250, 507-509.

(21) Hurwitz, H. D.; Dibiani, R. Experimental and theoretical investigations of steady and transient states in systems of ion exchange bipolar membranes, Journal of Membrane Science 2004, 228, 17-43.

(22) Daiguji, H.; Oka, Y.; Shirono, K. Nanofluidic Diode and Bipolar Transistor, Nano Lett 2005, 5, 2274-2280.

(23) Plecis, A.; Schoch Reto, B.; Renaud, P. Ionic transport phenomena in nanofluidics: experimental and theoretical study of the exclusionenrichment effect on a chip, Nano Lett 2005, 5, 1147-1155.

(24) Pu, Q.; Yun, J.; Temkin, H.; Liu, S. Ion-Enrichment and Ion-Depletion Effect of Nanochannel Structures, Nano Lett 2004, 4, 1099-1103.

(25) Razunguzwa, T. T.; Timperman, A. T. Fabrication and Characterization of a Fritless Microfabricated Electroosmotic Pump with Reduced $\mathrm{pH}$ Dependence, Anal Chem 2004, 76, 1336-1341.

(26) Chatterjee, A. N.; Cannon, D. M.; Gatimu, E. N.; Sweedler, J. V.; Aluru, N. R.; Bohn, P. W. Modeling and Simulation of Ionic Currents in ThreeDimensional Microfluidic Devices with Nanofluidic Interconnects, Journal of Nanoparticle Research 2005, 7, 507-516.

(27) Nischang, I.; Chen, G.; Tallarek, U. Electrohydrodynamics in hierarchically structured monolithic and particulate fixed beds, J Chromatogr A 2006, 1109, 32-50.

(28) Rubinstein, I.; Zaltzman, B. Electro-osmotically induced convection at a permselective membrane, Physical Review E: Statistical Physics, Plasmas, Fluids, and Related Interdisciplinary Topics 2000, 62, 22382251. 


\section{Chapter 3}

\section{Investigation of Zone Migration in a Current Rectifying Nanofluidic/Microfluidic Analyte Concentrator}

This chapter was previously published by Kathleen Kelly (Reschke), Scott A. Miller, and Aaron T. Timperman and appears here with minor changes. (Analytical Chemistry, 2009, 81, 732-738).

\section{Abstract}

A simple microfluidic device that uses a nanocapillary membrane (NCM) to connect a microfluidic channel and solution reservoir is capable of rectifying ionic current and enrichment of ionic species. Application of a potential induces concentration polarization (CP), which creates ion depleted and ion enriched zones on opposite sides of the permselective NCM. An enriched zone forms at the interface of the bulk buffer solution and depleted CP zone in the "off" state or the low current case. After polarity reversal, the migration of an enriched zone of anionic tracer is imaged. By decreasing the volume of the solution at the microchannel and NCM interface, the response time of the current rectifier is decreased and elution of the zone of anionic tracer is achieved. The decrease in response time is most dramatic for the "on" to "off" state transition. For this transition, the response time decreases from $\sim 50 \mathrm{~s}$ to $\sim 1 \mathrm{~s}$. The decrease in response time for the "off" to "on" state is not as dramatic and is characterized by the time from polarity reversal to current peak, which decreased from $84 \mathrm{~s}$ to 21 s. The features in the I-t plots can be accounted for with schematics of the zone migration that show the migration of depleted concentration polarization and enriched CP zones. Together the fluorescent images and the l-t plots provide the foundation for schematics that describe the zone elution following polarity reversal. These results provide an improved understanding of the zone migration and current rectification in nanofluidic-microfluidic interfaces with symmetric nanochannels. 


\subsection{Introduction}

Microfluidic systems have many characteristics that will enable them to aid in the advancement of high-throughput analyses through integrated sample preparation, massive parallelism, and rapid analysis times. While these devices are well suited for high-throughput analyses, detection can be challenging due to their micron sized channel dimensions and small volume samples present. Nanofluidic elements are being incorporated into microfluidic devices to improve device functionality, and such devices can be referred to as nanofluidicmicrofluidic interfaces (NMls). To date, nanofluidic elements have been integrated into microfluidic systems to enrich sample concentration, ${ }^{1-12}$ for valving as molecular gates, ${ }^{13-15}$ and for ionic current rectification. ${ }^{16,17}$

The extra functionality gained through the incorporation of nanofluidic elements into microfluidic devices, arises from their unique mass transport properties. By fabricating nanochannels with widths or radii smaller than the Debye length, the double layer overlaps within the channel, resulting in an ion selective or permselective channel. ${ }^{18}$ This permselectivity was elegantly illustrated experimentally in metal coated nanotubule membranes whose surface charge could be controlled by the applied potential. ${ }^{19}$ Plecis et al demonstrated both experimentally and theoretically that double layer overlap causes the nanochannel to be ion permselective ${ }^{20}{ }^{21}$

NMls are generally formed by etching or machining nanochannel(s) directly into the substrate or by joining a nanocapillary membrane (NCM) to a microfluidic channel. Nanochannels are typically larger (30 to $100 \mathrm{~nm})$ than the rather straight and uniform nanocapillaries of NCMs (10 nm). Smaller nanocapillaries and nanochannels allow for the use of higher buffer concentrations while maintaining double layer overlap. Nanoporous materials have also been fabricated in microfluidic channels by methods such as, polymerization of gels,,${ }^{6,7}$ porous silicates, ${ }^{8,9}$ and laser patterned microdialysis membranes. ${ }^{4,22}$

To the best of our knowledge, the first report of using an NMI for enrichment of analyte concentration utilized a concentrator with microfluidic 
channels patterned in polydimethylsiloxane (PDMS) and an NCM incorporated into the channel network. ${ }^{1}$ The NCM connected two microfluidic channels, and through the application of a potential sample ions with the same charge as the nanocapillary surface (co-ions) concentrated at the NCM. Upon reversal of the polarity, the enriched zone of sample was eluted.

Several other analyte concentrators have been reported that utilize the permselectivity of nanochannels and the formation of a zone of enriched analyte concentration at NMls. A device reported by Wang et al incorporated a set of nanochannels that connected two microchannels that were horizontally separated. ${ }^{2}$ They observed the development of a depleted CP zone and utilized it to achieve the highest reported concentration factors $\left(10^{6}\right.$ to $\left.10^{8}\right)$ for such devices. Kim et al reported concentration factors as high as $10^{6}$ in 30 min with a device fabricated of glass and PDMS, which is believed to form a 20 micron long nanochannel between weakly bonded PDMS and glass. ${ }^{10}$ They were skeptical of their reported concentration factors, because the measured flow rates were not large enough to deliver the requisite amount of sample. A different group investigated the relationship between EOF of the second kind, concentration polarization, and limiting current behavior to better understand the transport of ions at NMIs. ${ }^{23}$ Current versus voltage plots were presented with Ohmic current, limiting current, and overlimiting current regimes similar to those reported at larger ${ }^{21}$ permselective membranes. Dhopeshwarkar et al studied a hybrid PDMS/glass device with a nanoporous structure photopolymerized in the middle of a channel. They also imaged the concentration polarization, and modeled the local electric fields and concentration profiles along the length of the channel. ${ }^{24}$ By comparing a neutral and charged hydrogel, they concluded that the neutral hydrogel is more efficient, because the depleted CP zone does not form in the microchannel, which reduces the voltage drop in other regions of the device and increases sample delivery. ${ }^{12}$

Concentration polarization $(\mathrm{CP})$ is a well established phenomenon that occurs when an ion selective material is placed in an electric field. As counterions pass through the permselective medium the co-ion transport is hindered, 
creating an enriched zone of co-ions on one side of the permselective material and a depleted zone on the other. The hindered transport of co-ions is caused by the large Debye length to nanochannel width ratio that causes the system to be at or near double layer overlap. Nanochannels fabricated in a microfluidic device were first reported to be permselective and produce CP by Pu et al who observed the development of the depleted CP and enriched CP zones on opposite sides of the nanochannels that was referred to as the ion-enrichment and ion-depletion effect. ${ }^{3}$

Previously, rectification of ionic current in fluidic systems has been achieved using asymmetric nanopores. ${ }^{25}$, and differentially coated ${ }^{16}$ nanochannels. Daiguji et al reported theoretical work determining that differentially coated nanochannels are expected to rectify ionic current. ${ }^{26}$ Ionic current rectification was achieved with these devices, incorporating differentially coated nanochannels that connect to a microfluidic channel on either side. ${ }^{16}$ This method of achieving ionic current rectification can be likened to methods that resulted in current rectification within larger bipolar membranes. ${ }^{27-29}$ Recently, we have reported the rectification of ionic current with a device that has symmetric nanocapillaries and asymmetry in the microfluidic system. ${ }^{17}$

In this report, the current response of the microfluidic ionic current rectifier is shown as a function of time following switching of the polarity of the applied potential. By minimizing the solution volume on the microfluidic channel side of the NCM, the response time of the device is decreased. The time to move from the "on" steady state current to the "off" steady state current is reduced significantly from $\sim 50 \mathrm{~s}$ to $\sim 1 \mathrm{~s}$ by decreasing the volume at the $\mathrm{NCM}$ /microfluidic channel interface. Fluorescent images of the concentration and elution of the enriched zone of an anionic tracer, fluorescein, provide direct evidence for the order of zone migration. The features in the l-t plots can be explained with, and provide additional support for, schematics showing the zone migration of the depleted CP and enriched CP zones. It is anticipated that an improved understanding of the mechanisms of ionic current rectification and zone migration following polarity reversal at an NMI concentrator will lead to the 
production of robust devices that will be used for many applications in proteomics and other fields.

\subsection{Methods}

\subsubsection{Fabrication of Glass Microfluidic Devices}

All of the microfluidic devices were fabricated using soda lime glass substrates from Telic (Valencia, CA) and were patterned using standard photolithography and wet chemical etching techniques described elsewhere. ${ }^{30}$ The microfluidic chips contained a $2 \mathrm{~cm}$ long microchannel $210 \mu \mathrm{m}$ across and $12 \mu \mathrm{m}$ in depth and an access hole at either end as shown in Figure 3.1A. For microfluidic devices with the top-mounted reservoir as shown in Figure 3.1A, the vertical $150 \mu \mathrm{m}$ ID access holes were drilled using150 $\mu \mathrm{m}$ drill bits from Kyocera Tycom Corporation (Irvine, CA). The patterned wafer was subsequently bonded to a second blank wafer by cleaning each slide chemically and with a high-

pressure wash, and placing them together and thermally bonding them. ${ }^{30}$ 

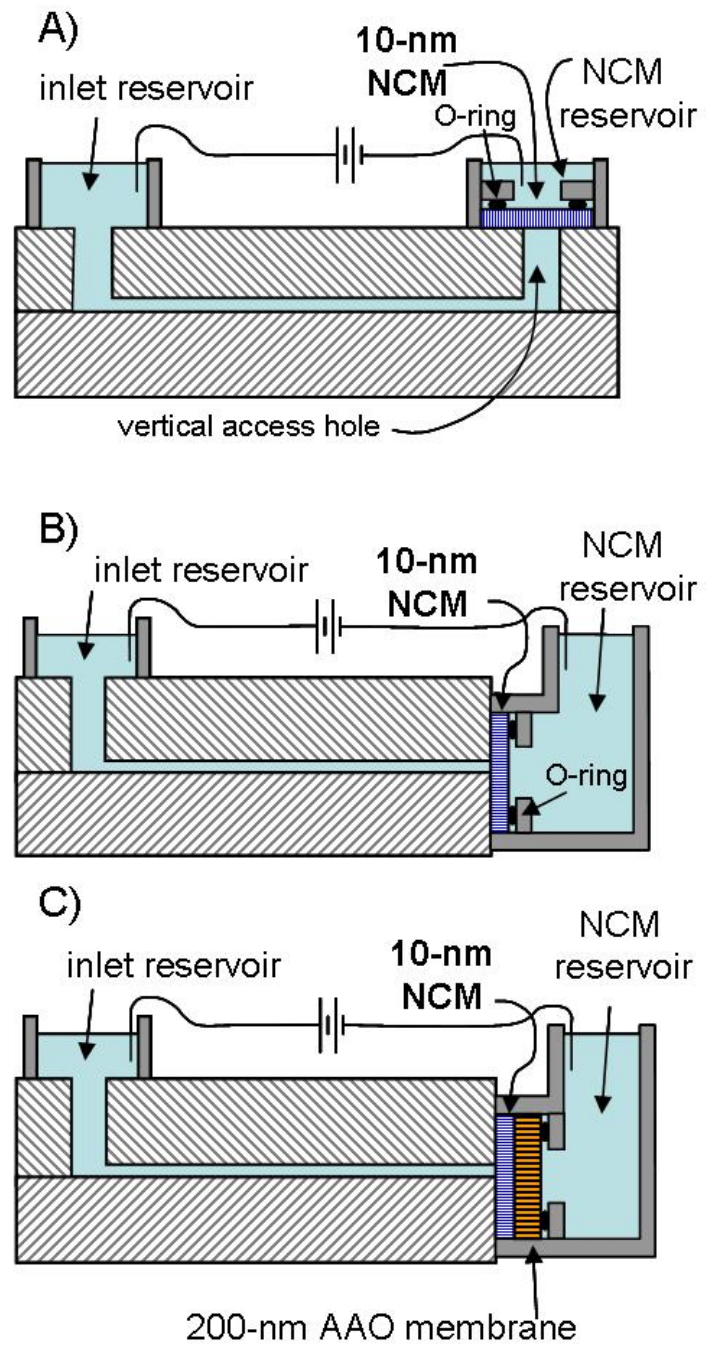

Figure 3.1: Three device designs are shown for the NMI with the PC-NCM. In the first design (A), the PC-NCM is attached to the top of the microfluidic device and a vertical channel with a cross-sectional area that is 8-fold greater than the horizontal channel. Thus, this design (A) creates a larger fluid volume at the NCM/microfluidic channel interface. The change in crosssectional area was eliminated in the second design B) by attaching the NCM directly to the end of the microfluidic channel. In $\mathrm{C}$ ) the volume at the $\mathrm{NCM}$ /microfluidic interface is further decreased by compressing it against the microchannel orifice by backing it with a rigid alumina membrane. 
For fabrication of the devices with the NCM directly at the end of the microfluidic channel shown in Figures 3.1B \& 3.1C, a reservoir is attached to the side of a microfluidic chip to incorporate the NCM. The end of the device was removed with a silicon carbide glass-cutting saw and Crystalbond (Aremco Products, Valley Cottage, NY) was used to protect the channel in a similar manner to Bings et al. ${ }^{31}$ Strips of glass plate (1/8" thick) were glued on each face of the microchip to attach the NCM-interface fitting. To allow a better seal with the NCM, the edge of the microchip was polished to smoothness using lapping film starting at $40 \mu \mathrm{m}$ and ending with the finest lapping film, $0.3 \mu \mathrm{m}$. The Crystalbond was remelted and removed by vacuum prior to flushing with acetone (Fisher Scientific), $1 \mathrm{M}$ hydrochloric acid (Fisher Scientific), and $1 \mathrm{M}$ sodium hydroxide (Fisher Scientific).

\subsubsection{Integration of NCMs and Device Preparation}

An Upchurch Scientific (Oak Harbor, WA) nanoport reservoir was attached at the inlet reservoir access hole and a black Delrin reservoir was attached as the NCM reservoir. ${ }^{17}$ Prior to each experiment, the microchannel was rinsed with 0.1 $M$ sodium hydroxide, deionized water, and the electrolyte solution which was 10 $\mathrm{mM}$ sodium phosphate monobasic (Fisher Scientific) at $\mathrm{pH}$ 7. The microchannel was filled with phosphate buffer prior to sealing the NCM to the microfluidic channel. The NCM was inserted into the housing and the reservoir and compressed against the glass device by compressing an o-ring (size 001 nominal ID and OD of $0.79 \mathrm{~mm}$ and $2.77 \mathrm{~mm}$, respectively). $10 \mathrm{~nm}$ track-etched polycarbonate (PC) NCMs from GE Osmonics Inc. (Minnetonka, MN) were used. For the device in Figure 3.1C, aluminum oxide membranes with $200 \mathrm{~nm}$ pores from Whatman (Sanford, Maine) cut to size with a razor blade were used to compress the polycarbonate NCM against the end of the microfluidic channel as shown in Figure 3.1C. The reservoirs were filled with buffer in a manner that ensured air bubbles were not present and the levels were equal. The platinum electrodes were placed in the reservoirs that were covered to minimize evaporation of solutions. 


\subsubsection{Current Measurement}

Potentials were applied with a TREK (Medina, NY) high-voltage power supply model $610 \mathrm{E}$. The current data was continuously recorded using a PCinterfaced digital multimeter and Meterview version 1.0. The current was measured with and without the NCM integrated into the microfluidic device. A computer program made with Labwindows controlled the TREK power supply, which was switched between $-800 \mathrm{~V}$ and $+800 \mathrm{~V}$ at a time interval of $600 \mathrm{~s}$ for Figures 3.4A \& 3.4B. Figures 3.4A \& 3.4B were collected in triplicate. To determine the time required to switch from the "on" state to the "off" state, the "off" steady state current was used as the baseline and the peak width at half height was measured. The "on" state current is less stable making it difficult to consistently measure a peak width at half height. Therefore, the "off" state to the "on" state transition was characterized by measuring the time between when the NCM electrode voltage is switched from $-800 \mathrm{~V}$ to $+800 \mathrm{~V}$ and the current max.

\subsubsection{Fluorescein Imaging}

The concentration process was imaged using a TE-2000U Eclipse Nikon (Melville, NY) inverted fluorescence microscope with a $2 x$ objective and a charged coupled device from Roper Scientific (Tucson, AZ) model 650. Images were processed with MetaMorph software version 6.2r6. For imaging the enriched zone, the $20 \mu \mathrm{M}$ fluorescein dye (Sigma Aldrich) was added to the 10 $\mathrm{mM}$ sodium phosphate monobasic buffer. After the device was filled with buffer solution and just prior to the application of the voltage, the buffer solution in the inlet reservoir was replaced with fluorescein solution. Therefore, fluorescein movement is from the inlet reservoir and toward the NCM during concentration enrichment. The images were flat field corrected and background subtracted.

\subsection{Results and Discussion}

NMIs that have an asymmetric fluidic geometry have been shown to rectify ionic current as previously reported. ${ }^{17}$ Ionic current rectification in this system is 
noteworthy, because it is achieved without the use of conical nanocapillaries ${ }^{25,32}$ or differentially coated nanocapillaries. ${ }^{16,26}$ In these systems, the electric field drives concentration polarization (CP) at a permselective NCM, creating zones of ion enrichment and ion depletion on opposite sides of the NCM. The "off" state is observed when the depleted CP zone forms in the microchannel, and the "on" state is observed when the enriched CP zone forms in the microchannel. As a result of $\mathrm{CP}$ creating depleted and enriched $\mathrm{CP}$ zones on opposite sides of the membrane, an enriched zone forms in the microchannel when the depleted CP zone forms in the microchannel. In this paper, the term enriched zone refers to the enriched zone that forms at the interface of the bulk buffer solution and the depleted CP zone and is distinct from the enriched CP zone. When imaged this same zone is referred to as the enriched zone of fluorescein, but the enriched analyte need not be fluorescein although it must be anionic because the NCM is cation permselective. The enriched zone of fluorescein forms at the edge of the $\mathrm{CP}$ depleted zone where the balancing of electrophoretic and EOF flow occurs.

\subsubsection{Changing Volume at the NCM/Microfluidic Channel Interface}

Here, three different microfluidic device designs are used to investigate the effects of changing the solution volume at the microchannel/NCM interface. Decreasing the solution volume adjacent to the NCM is found to decrease the response time of current rectification. Features in the l-t curves can be correlated with fluorescent images of the migration of an enriched zone of fluorescein, an anionic dye.

In our first microfluidic device design, shown in Figure 3.1A, the horizontal channel has a $210-\mu \mathrm{m} \times 12-\mu \mathrm{m}$ cross-section terminated with a $150-\mu \mathrm{m}$ diameter access hole that leads to the NCM. The cross-sectional area of the vertical channel is about 8-fold greater than the horizontal channel, which creates a relatively larger solution reservoir immediately in front of the NCM. In order to eliminate the change in cross-sectional area, a second design is used that interfaces the NCM directly with the microchannel by connecting the NCM directly to the microchannel end as shown in Figure 3.1B. However, because the 
PC-NCM is not strongly held against the microchannel, there remains a small excess volume of solution between the PC-NCM and the microfluidic device. As shown in Figure 3.1C, the third design eliminates the extra volume between the PC-NCM and the microchannel by supporting the PC-NCM with a rigid anodized aluminum oxide (AAO) membrane with larger pores. In order to minimize alterations of the nanofluidic system, a rigid AAO membrane with relatively large 200-nm pores is used that does not exhibit ion permselectivity with these buffer systems.

\subsubsection{Fluoresence Imaging}

In the "off" state, a depleted CP zone forms in the microchannel and adjacent to the NCM, while a zone of concentrated fluorescein forms at the interface between the depleted CP zone and the bulk buffer solution. ${ }^{21,33,34}$ This enriched zone at the depleted CP zone and bulk buffer solution interface has been shown to be caused by the balancing between the EOF and the reverse electrophoretic mobility that increases for anions as they enter the depleted CP zone and approach the permselective material. ${ }^{12}$ As shown in Figure 3.2, the intensity of the enriched zone of fluorescein increases and its distance from the NCM increases as the voltage is increased. Because the enriched zone of fluorescein forms at the interface of the depleted CP zone and the bulk buffer solution where the EOF and opposing electrophoretic mobility balance, its movement away from the NCM indicates that the depleted CP zone expands as the voltage is increased. Expansion of the depleted CP zone with increasing voltage is consistent with the hypothesis that the overall resistance of the system in the "off" state is governed by the formation of the depleted CP zone in the microfluidic channel. 


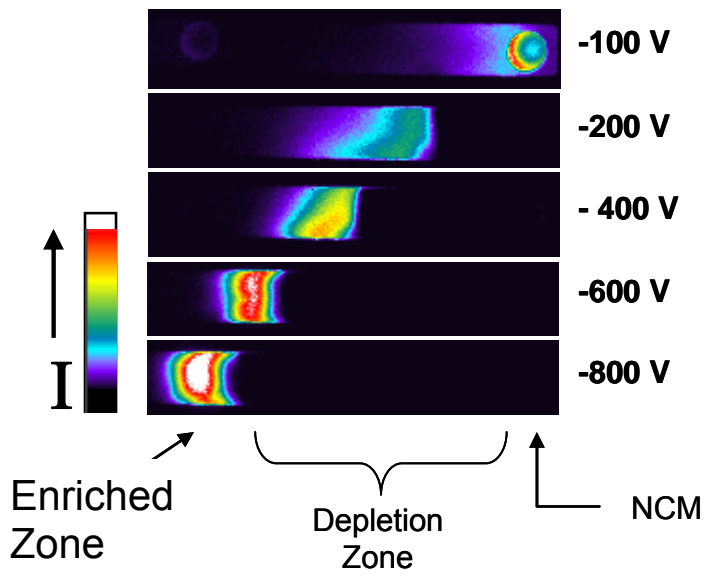

Figure 3.2: Images of the enriched zone of fluorescein in the microchannel of the device design shown in Figure 3.1A. An enriched zone of fluorescein develops between the bulk solution and the depleted CP zone, and therefore its position is indicative of the length of the depleted CP zone. The images reveal that the length of the depleted CP zone and the concentration of the enriched zone of fluorescein both increase as the magnitude of the voltage applied in the "off" state is increased. A constant spatial alignment of the images with respect to the position of the vertical NCM channel is maintained. The enriched zone of fluorescein is mostly contained within the access hole at $-100 \mathrm{~V}$; at $-200 \mathrm{~V}$ the $\mathrm{CP}$ ion depletion zone has grown and enriched zone of fluorescein is outside the vertical NCM channel; and at $-400 \mathrm{~V}$ through $-800 \mathrm{~V}$ the depleted CP zone continues to grow in length while the intensity of the enriched zone of fluorescein increases.

Additional insight is gained by rapidly imaging the migration of the enriched zone of fluorescein in the third design (Figure 3.1C) as the system is switched from the "off" to the "on" state by changing the polarity of the potential applied to the NCM reservoir. In Figure 3.3, an enriched zone of fluorescein is shown in the first panel that is similar to the enriched zone of fluorescein shown in Figure 3.2. During the second frame shown in Figure 3.3, the polarity is switched and the enriched zone of fluorescein is shown to stream through the depleted $\mathrm{CP}$ zone and toward the NCM. In the third frame, the concentrated fluorescein zone has migrated to the face of the NCM and out of the field of view. 


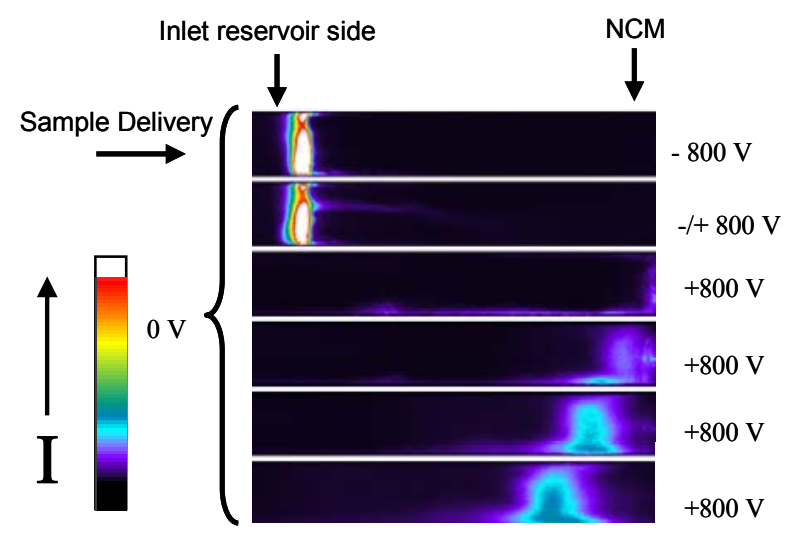

Figure 3.3: Fluorescent images showing the elution of an enriched zone of fluorescein in an NMI with the alumina backed side interface design (Figure 3.1C). Initially (frame 1), the enriched zone of fluorescein is shown in the well developed "off" state with $0 \mathrm{~V}$ applied to the inlet reservoir, and $-800 \mathrm{~V}$ applied to the NCM reservoir. During, frame 2 , the polarity is switched and $+800 \mathrm{~V}$ is applied to the NCM reservoir. In frame 3, the enriched zone of fluorescein has migrated through the depleted CP zone and toward the face of the NCM that is just outside of the viewable area. In frames 4 through 6 , the enriched zone of fluorescein moves back into the field of view and towards the inlet reservoir indicating that EOF is dominant.

The high electric field in this depleted CP zone increases the speed of ion migration and causes it to migrate through this region in less than one frame (0.2-s). Additionally, the high electric field across the depleted CP zone reduces the electric field in other regions of the system. Therefore, the rapid migration of the enriched zone of fluorescein through the depleted CP zone provides additional confirmation that the concentration of ions is greatly reduced in this zone. After the enriched zone of fluorescein has migrated through the depleted $\mathrm{CP}$ zone, the resistance of the solution and the electric field are decreased. The decreased electric field strength causes a decrease in the contribution of the electrophoretic mobility to the observed velocity. Next, the enriched zone of fluorescein moves away from the surface of the NCM and back into the field of view, indicating EOF has become dominant. In the last three frames, EOF continues to move the enriched zone of fluorescein moves away from the NCM and towards the inlet reservoir. These images were obtained with the device design shown in Figure 3.1C, which has the smallest volume at the microchannel/NCM interface. With the devices shown in Figures 3.1A \& 3.1B, 
dilution of the enriched zone of fluorescein due to larger volumes of solution at the interface resulted in inefficient elution.

\subsubsection{I-t Plots}

The l-t plots for the different device designs were generated by switching the polarity of the potential applied to the NCM reservoir between negative and positive. I-t plots for each design have the same general appearance, but differences in their features are also observed as shown in Figure 3.4. Current generated by applying a positive potential to the NCM reservoir is defined as positive, and the current generated when a negative potential is applied to the NCM reservoir is defined as negative. In each of the three I-t plots various temporal features are observed, prior to current reaching a quasi-steady-state. In Figure 3.4, the dashed line indicates the value of the current observed when no NCM is present in the system. The quasi-steady-state current observed when a negative potential is applied to the NCM reservoir is much lower than the current observed when a positive potential is applied to the NCM reservoir, creating the "off" and "on" states respectively. 

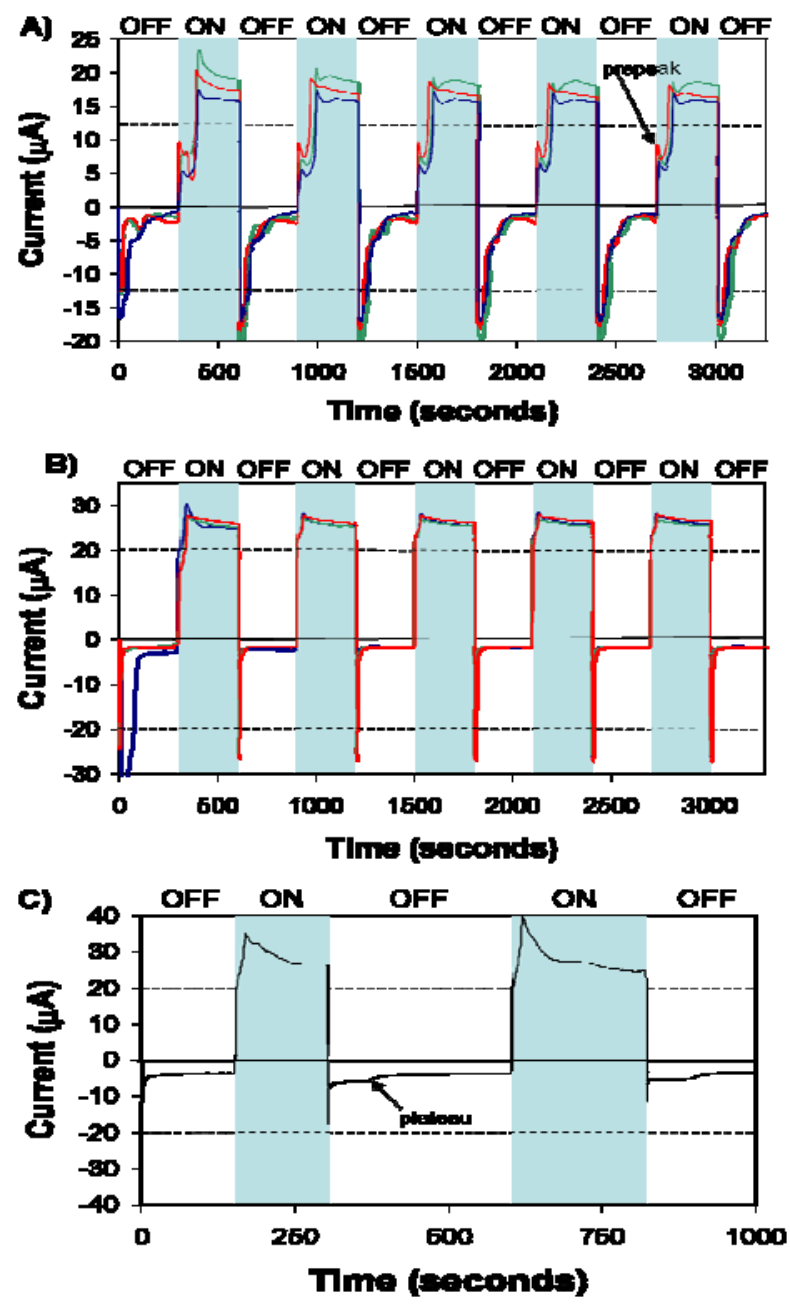

Figure 3.4: The current responses as a function of time are shown for the corresponding device designs shown in Figure 3.1A, 3.1B, and 3.1C as each device is switched between the "on" and the "off" states, by applying $+800 \mathrm{~V}$ and $-800 \mathrm{~V}$ at the NCM reservoir respectively. The "off" state yields lower quasi-steady state currents than the "on" state. In A), the top-mounted NCM device has the widest peaks and longest times to approach a steady state current. In $B$ ) the reduced volume with the side mounted PC-NCM decreases the rise time and peak widths. In C), the interface volume is further decreased and peak widths are decreased further as well. 


\subsection{4 "Off" to "On" State Transition}

When the "off" to "on" state transition or polarity reversal from negative to positive is examined, little decrease is noted in the time required to reach steady state current with changes in device design. However, the deviation of the current response from a square wave is reduced as the volume in front of the NCM is reduced. The additional transient features that follow switching from the "off" to "on" state, provide features that are consistent with the schematics presented in Figure 3.5. 


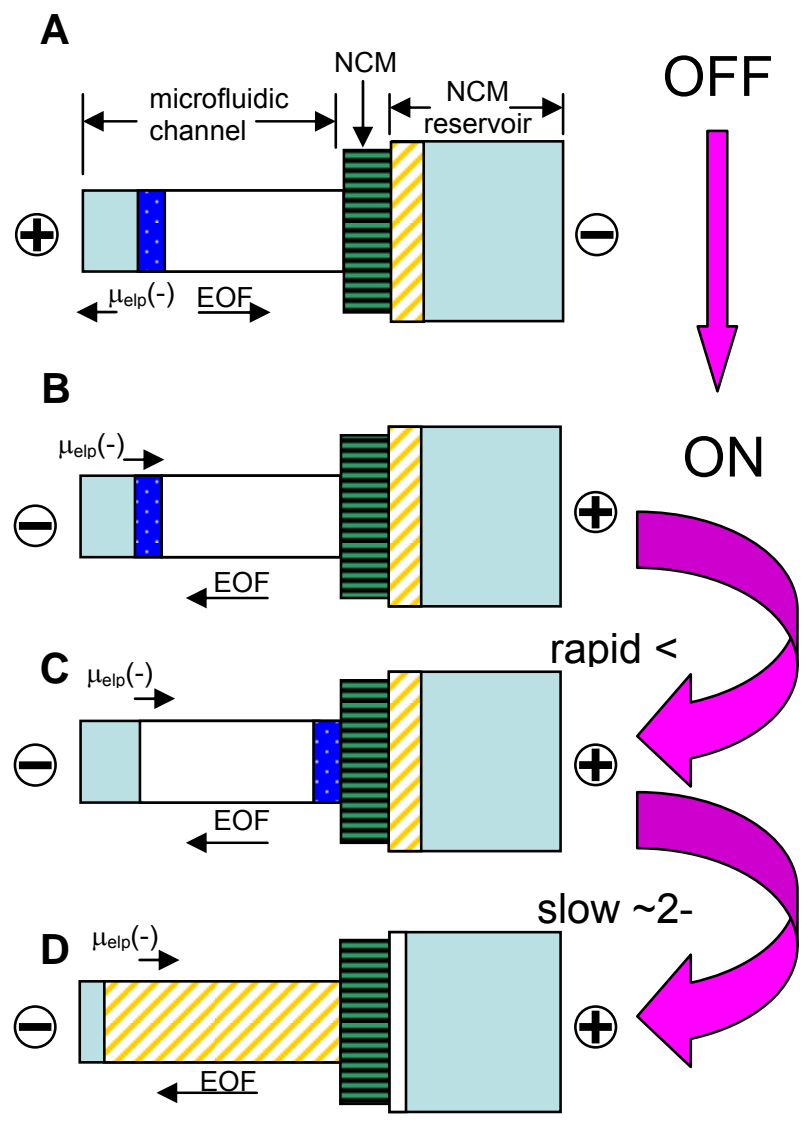

$$
\begin{aligned}
& \therefore \text { enriched zone } \\
& \text { CP enriched zone } \\
& \text { CP depletion zone } \\
& \text { bulk buffer solution }
\end{aligned}
$$

Figure 3.5: A schematic representation of the enriched zone and CP zones following the "off" to "on" state transition. In A), the fully developed "off" state is shown prior to switching of the potential. In B), the potentials and direction of EOF and electrophoretic mobilities are shown immediately after the switching to the "on" state. Between B) and C), the enriched zone rapidly migrates through the depleted CP zone and toward the face of the NCM. After the enriched zone passes through the depleted CP zone, it is swept out of the microfluidic channel by EOF as shown in $\mathrm{C}$ ). As the zones migrate toward the inlet reservoir and out of the microchannel, the system is transitioning to the fully developed "on" state shown in D).

The top interface chip (Figure 3.1A) has the greatest volume in front of the NCM, and shows the most pronounced features in Figure 3.4A. Upon polarity reversal, the enriched zone in the microchannel rapidly migrates across the depleted $\mathrm{CP}$ 
zone and toward the NCM surface producing the orientation shown in Figure 3.5C. After the enriched zone has migrated through the ion depletion zone, EOF reverses the direction of the enriched zone, pumping it away from the NCM and toward the inlet reservoir with the depleted CP zone preceding the enriched zone. While the high resistance depleted CP zone remains in the microchannel, the pre-peak and plateau following the pre-peak are observed in the I-t plot. As the depleted CP zone is pumped out of the microchannel, the conductivity of the microchannel increases and the current increases, producing the main peak observed in Figure 3.4A. This main peak is smaller, but still observed in Figures 3.4B \& C. The peak current occurs when the enriched zone remains in the microchannel, and the current diminishes as it is pumped out of the microchannel, producing the decay to the quasi-steady-state value. After these zones are pumped out of the microchannel by EOF, the system has transitioned to the "on" steady state (Figure 3.5D), and a long zone of slightly enriched concentration has formed in the microchannel. The concentration enhancement of this CP enriched zone is thought to be small due to the opposing EOF that pumps rapidly toward the inlet reservoir.

\subsection{5 "On" to "Off" State Transition}

The I-t plots show a decrease in the response time for the "on" to "off" state transition as the volume of the solution immediately in front of the NCM is decreased. The device design shown in Figure 3.1A has the largest volume in front of the NCM, and consequently yields the slowest response time, requiring $\sim 50 \mathrm{~s}$ before the current reaches a steady state in Figure 3.4A. The volume in front of the NCM is reduced in the device shown in Figure 3.1B, and the time required to reach the quasi-steady state current is decreased to about $7 \mathrm{~s}$ as seen in Figure 3.4B. In Figure 3.4C, the initial current spike associated with the "on" to "off" state transition is reduced to only $1 \mathrm{~s}$ in width. The rapid reduction in current is indicative of the rapid formation of a depleted CP zone in the microchannel following this transition. The reduction in volume at the $\mathrm{NCM} /$ microchannel interface achieved with the device shown in Figure 3.1C 
allows the depleted CP zone to rapidly extend into the microchannel and reduce the current. Similar current behavior characterized by a high initial current decaying to a near steady-state value was first reported by Dhopeshwarkar et al. ${ }^{24}$ This decay profile is caused by the growth and increased resistance of the depleted CP zone until a near steady state is reached. The initial current spike of the "on" to "off" transition can be considered to arise from a capacitive effect in which the potential reversal causes rapid migration of the ions accumulated in the previous state. A zone with a small enrichment in concentration is thought to form in the microchannel during the "on" state as shown in the schematics Figure 3.6A.

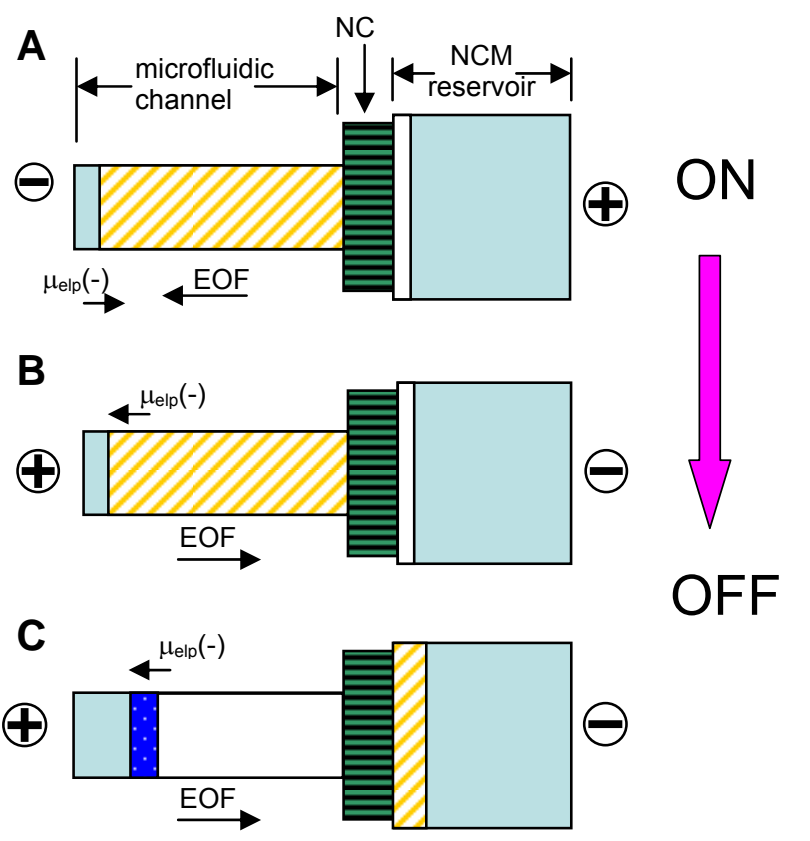

Figure 3.6: A schematic representation of the "on" to "off" state transition is shown. In A), the fully developed "on" state is shown prior to switching of the potential. In B), the potentials and direction of EOF is shown immediately after switching to the "off" state polarity. Between B) and C), the EOF is thought to drive cations of the enriched CP zone through the NCM, producing the "off" state current plateau shown in Figure 3.4. In C), the enriched zone and depleted CP zone are shown for the fully developed "off" state. The color scheme is the same as Figure 3.5.

In the "on" state, EOF prevents the migration of fluorescein into the microchannel from the inlet reservoir. However, the buffer anions, hydrogenphosphate and dihydrogen phosphate ion, have electrophoretic mobilites of $7.15 \times 10^{-4} \mathrm{~cm}^{2} \mathrm{~V}^{-1} \mathrm{~s}^{-1}$ and $5.91 \times 10^{-4} \mathrm{~cm}^{2} \mathrm{~V}^{-1} \mathrm{~s}^{-1}$, respectively. ${ }^{35}$ Therefore, the electrophoretic mobilities 
of major buffer ions exceed the electrophoretic mobility of fluorescein $2.92 \times 10^{-4}$ $\mathrm{cm}^{2} \mathrm{~V}^{-1} \mathrm{~s}^{-1},{ }^{36}$ making it possible for enrichment of these ions even when the opposing EOF is too great to allow fluorescein enrichment.

In Figure 3.4C, a current plateau is observed in the second and third "off" state excursions that is hypothesized to result from the formation of an enriched $\mathrm{CP}$ zone during the "on" state that creates a slight concentration enhancement in the microchannel. The increase in concentration is thought to be slight due to the small current offsets it produces and the opposing EOF. This enriched CP zone elutes in $\sim 100 \mathrm{~s}$, as shown by the disappearance of the current plateau as it reaches steady state current. Consistent with this hypothesis, is the absence of this current plateau in the first negative excursion shown in Figure 3.4C. This first negative excursion was not preceded by the "on" state and consequently the enriched CP zone is absent from the microchannel, eliminating this feature.

\subsection{Conclusion}

An ionic current rectifier has been fabricated by placing an NCM between a microfluidic channel and a macroscopic reservoir and the processes that occur as it is transitioned between its "on" and "off" states are investigated. By minimizing the volume directly in front of the NCM, a faster response time is achieved for the transitions between the "on" and "off" states and elution of an enriched zone of fluorescein is achieved. Images of the enriched zone of fluorescein at the bulk buffer and depleted CP zone interface indicate that the length of the depleted CP zone increases as the applied voltage is increased. Elution of the enriched zone is achieved by minimizing the volume at the microchannel NCM interface and reversing the potential; however, the enriched zone migrates rapidly through the depleted $\mathrm{CP}$ zone before the EOF sweeps it towards the inlet reservoir. The l-t plots exhibit reproducible current spikes that eventually relax to a quasi-steady state current that are explained through schematics based on fluorescent images of an anionic dye as it migrates through the microchannel as the device is transitioned between the "on" and "off" states. The "on" state currents that are elevated compared to the no NCM current were 
previously explained by a long enriched CP zone forming in front of the NCM, which is consistent with the explanation of the l-t plot features. Together, the fluorescent images of the migration of the enriched zone of fluorescein and the I-t plots provide support for the schematics that describe the elution of the enriched zone as the polarity is reversed. 


\subsection{References}

(1) Zhang, Y.; Timperman, A. T. Integration of nanocapillary arrays into microfluidic devices for use as analyte concentrators, Analyst (Cambridge, United Kingdom) 2003, 128, 537-542.

(2) Wang, Y.-C.; Stevens, A. L.; Han, J. Million-fold Preconcentration of Proteins and Peptides by Nanofluidic Filter, Anal Chem 2005, 77, 42934299.

(3) Pu, Q.; Yun, J.; Temkin, H.; Liu, S. Ion-Enrichment and Ion-Depletion Effect of Nanochannel Structures, Nano Lett 2004, 4, 1099-1103.

(4) Song, S.; Singh Anup, K.; Kirby Brian, J. Electrophoretic concentration of proteins at laser-patterned nanoporous membranes in microchips, Anal Chem 2004, 76, 4589-4592.

(5) Hatch, A. V.; Herr Amy, E.; Throckmorton Daniel, J.; Brennan James, S.; Singh Anup, K. Integrated preconcentration SDS-PAGE of proteins in microchips using photopatterned cross-linked polyacrylamide gels, Anal Chem 2006, 78, 4976-4984.

(6) Dai, J.; Ito, T.; Sun, L.; Crooks, R. M. Electrokinetic trapping and concentration enrichment of DNA in a microfluidic channel, Journal of the American Chemical Society 2003, 125, 13026-13027.

(7) Dhopeshwarkar, R.; Sun, L.; Crooks, R. M. Electrokinetic concentration enrichment within a microfluidic device using a hydrogel microplug, Lab on a Chip 2005, 5, 1148-1154.

(8) Khandurina, J.; Jacobson, S. C.; Waters, L. C.; Foote, R. S.; Ramsey, J. M. Microfabricated Porous Membrane Structure for Sample Concentration and Electrophoretic Analysis, Anal Chem 1999, 71, 1815-1819.

(9) Foote, R. S.; Khandurina, J.; Jacobson, S. C.; Ramsey, J. M. Preconcentration of proteins on microfluidic devices using porous silica membranes, AnalChem 2005, 77, 57-63.

(10) Kim Sun, M.; Burns Mark, A.; Hasselbrink Ernest, F. Electrokinetic protein preconcentration using a simple glass/poly(dimethylsiloxane) microfluidic chip, Anal Chem 2006, 78, 4779-4785.

(11) Lee, J. H.; Chung, S.; Kim, S. J.; Han, J. Poly(dimethylsiloxane)-Based Protein Preconcentration Using a Nanogap Generated by Junction Gap Breakdown, Anal Chem 2007, 79, 6868-6873.

(12) Hlushkou, D.; Dhopeshwarkar, R.; Crooks Richard, M.; Tallarek, U. The influence of membrane ion-permselectivity on electrokinetic concentration enrichment in membrane-based preconcentration units, Lab on a chip 2008, 8, 1153-1162.

(13) Kuo, T.-C.; Cannon Donald, M., Jr.; Chen, Y.; Tulock Joseph, J.; Shannon Mark, A.; Sweedler Jonathan, V.; Bohn Paul, W. Gateable nanofluidic interconnects for multilayered microfluidic separation systems, Anal Chem 2003, 75, 1861-1867.

(14) Gatimu, E. N.; King, T. L.; Sweedler, J. V.; Bohn, P. W. Three-dimensional integrated microfluidic architectures enabled through electrically switchable nanocapillary array membranes, Biomicrofluidics 2007, 1. 
(15) Tulock, J. J.; Shannon, M. A.; Bohn, P. W.; Sweedler, J. V. Microfluidic Separation and Gateable Fraction Collection for Mass-Limited Samples, Anal Chem 2004, 76, 6419-6425.

(16) Karnik, R.; Duan, C.; Castelino, K.; Daiguji, H.; Majumdar, A. Rectification of ionic current in a nanofluidic diode, Nano Lett 2007, 7, 547-551.

(17) Miller, S. A.; Kelly, K. C.; Timperman, A. T. Ionic current rectification at a nanofluidic/microfluidic interface with an asymmetric microfluidic system, Lab on a Chip 2008, 8, 1729-1732.

(18) Probstein, R. F. Physicochemical Hydrodynamics. An Introduction, 1989.

(19) Nishizawa, M.; Menon, V. P.; Martin, C. R. Metal nanotubule membranes with electrochemically switchable ion-transport selectivity, Science (Washington, D. C.) 1995, 268, 700-702.

(20) Plecis, A.; Schoch Reto, B.; Renaud, P. Ionic transport phenomena in nanofluidics: experimental and theoretical study of the exclusionenrichment effect on a chip, Nano Lett 2005, 5, 1147-1155.

(21) Rubinstein, I.; Zaltzman, B. In Surface Chemistry and Electrochemistry of Membranes; Sorensen, T. S., Ed., 1999; Vol. 79, pp 591-621.

(22) Song, S.; Singh, A. K.; Shepodd, T. J.; Kirby, B. J. Microchip dialysis of proteins using in situ photopatterned nanoporous polymer membranes, Anal Chem 2004, 76, 2367-2373.

(23) Kim, S. J.; Wang, Y.-C.; Lee, J. H.; Jang, H.; Han, J. Concentration polarization and nonlinear electrokinetic flow near a nanofluidic channel, Physical Review Letters 2007, 99, 044501/044501-044501/044504.

(24) Dhopeshwarkar, R.; Crooks Richard, M.; Hlushkou, D.; Tallarek, U. Transient effects on microchannel electrokinetic filtering with an ionpermselective membrane, Anal Chem 2008, 80, 1039-1048.

(25) Siwy, Z.; Heins, E.; Harrell, C. C.; Kohli, P.; Martin, C. R. ConicalNanotube Ion-Current Rectifiers: The Role of Surface Charge, Journal of the American Chemical Society 2004, 126, 10850-10851.

(26) Daiguji, H.; Oka, Y.; Shirono, K. Nanofluidic Diode and Bipolar Transistor, Nano Lett 2005, 5, 2274-2280.

(27) Lovrecek, B.; Despic, A.; Bockris, J. O. M. Electrolytic junctions with rectifying properties, Journal of Physical Chemistry 1959, 63, 750-751.

(28) Suendo, V.; Eto, R.; Tanioka, A. Ionic Rectification Properties of a Bipolar Interface Consisting of a Cationic Surfactant and Cation-Exchange Membrane, Journal of Colloid and Interface Science 2002, 250, 507-509.

(29) Hurwitz, H. D.; Dibiani, R. Experimental and theoretical investigations of steady and transient states in systems of ion exchange bipolar membranes, Journal of Membrane Science 2004, 228, 17-43.

(30) Razunguzwa, T. T.; Timperman, A. T. Fabrication and Characterization of a Fritless Microfabricated Electroosmotic Pump with Reduced $\mathrm{pH}$ Dependence, Anal Chem 2004, 76, 1336-1341.

(31) Bings, N. H.; Wang, C.; Skinner, C. D.; Colyer, C. L.; Thibault, P.; Harrison, D. J. Microfluidic devices connected to fused-silica capillaries with minimal dead volume, Anal Chem 1999, 71, 3292-3296. 
(32) Siwy, Z. S. Ion-current rectification in nanopores and nanotubes with broken symmetry, Advanced Functional Materials 2006, 16, 735-746.

(33) Spiegler, K. S. Polarization at ion exchange membrane-solution interfaces, Desalination 1971, 9, 367-385.

(34) Levich, V. G. Physicochemical Hydrodynamics; Prentice-Hall: Englewood Cliffs, NJ, 1962.

(35) Hirokawa, T.; Nishino, M.; Kiso, Y. Isotachophoretic determination of mobility and pKa by means of computer simulation. II. Evaluation of MO and pKa of 65 anions, J Chromatogr 1982, 252, 49-65.

(36) Gong, M.; Wehmeyer Kenneth, R.; Limbach Patrick, A.; Heineman William, R. On-line sample preconcentration by sweeping with dodecyltrimethylammonium bromide in capillary zone electrophoresis, $\mathrm{J}$ Chromatogr A 2006, 1125, 263-269. 


\title{
Chapter 4
}

\section{Development of an ON State Electrophoretic Dominant Concentrator Based on a Nanofluidic/Microfluidic Interface}

\author{
Abstract \\ Two nanofluidic/microfluidic concentrators are presented with low net \\ electroosmotic flow in which samples are delivered by electrophoresis. One \\ device is symmetric with microchannels of similar dimensions on opposite sides \\ of the nanofluidic channel, and the second device is asymmetric with a \\ microfluidic channel on the sample delivery side of the nanochannel and \\ macroscopic reservoir is on the outlet side. Both devices are capable of \\ enriching anionic sample and are electrophoretic dominant concentrators \\ meaning that they deliver sample via electrophoresis rather than via \\ electroosmotic flow. The symmetric device exhibits poor concentration factors of \\ only $\sim 7$. The asymmetric device has an improved concentration factor of $\sim 50$. \\ The asymmetry of the design causes the device to rectify current with a \\ rectification factor of $\sim 6.6$ at $\pm 100 \mathrm{~V}$. The $\mathrm{ON}$ state or high current state occurs \\ as the sample becomes enriched in the microchannel. Thus, the device can also \\ be considered an $\mathrm{ON}$ state electrophoretic concentrator. The higher $\mathrm{ON}$ state \\ current leads to the improved concentration factors.
}




\subsection{Introduction}

The field of proteomics has the potential to influence various medical fields by identifying proteins responsible for causing disease to proliferate throughout cells and eventually influencing the overall functioning of entire organs. Proteomic samples have a large complexity with great diversity stemming from the 20 possible amino acids that make up proteins. Additionally, the samples have a large dynamic range in which low abundance proteins are present at levels as low as a few copies per cell while high abundance proteins are present at levels as high as $10^{6}$ copies per cell. ${ }^{1}$ Sample contamination and loss are two issues that plague the field of proteomics as well, because, often the proteins of the most interest are at the lowest concentration and thus the most difficult to detect. The field still suffers from inadequate instrumentation to carry out the goals of proteomics.

Microfluidics offers a means to achieve parallel analyses of multiple samples with multiplexed sample preparation and separation techniques while minimizing sample contamination and loss. The sample complexity necessitates the use of the multidimensional separations to achieve the highest peak capacity possible. Developing microfluidic devices for application to proteomics is an area of much interest, because these techniques offer rapid separations and the ability to easily integrate several steps onto one device with limited sample loss and contamination.

While microfluidics are an attractive approach for proteomic analysis, one drawback is the extremely small volumes of sample involved that place a greater demand on detection methods. Therefore, an on-chip sample concentrator can increase the sample concentration and minimize the burden placed on the detection systems. Several on-chip concentrators in the literature integrate a nanofluidic element into the microfluidic system and rely on a phenomenon called concentration polarization (CP) for sample concentration. ${ }^{2-26} \mathrm{CP}$ arises from the ion permselectivity of the nanofluidic element when a voltage is applied. ${ }^{27}$ Permselectivity results from the material preferentially transporting either cations 
or anions and causes two zones to develop on either side of the nanofluidic element as the transport occurs. One of the zones is depleted of ions and is referred to as the CP depleted zone while the other zone is enriched with ions and is referred to as the enriched CP zone.

Nanofluidic microfluidic interfaces (NMIs) that rely on CP to enrich sample can be split into two separate categories: those that rely on EOF for sample delivery and those that rely on electrophoresis for sample delivery. The location of the enriched analyte is dependent on the level of EOF through the device as shown in Figure 4.1. All of the devices develop CP, but the devices that have high EOF enrich sample near the interface of the bulk buffer solution and CP depleted zone, and devices with low EOF enrich sample within the enriched CP zone as shown in Figure 4.1. The polarity is reversed between the EOF dominant and electrophoretic dominant concentrators to ensure sample delivery within both devices. While most of the literature provides the necessary information to determine whether EOF or electrophoresis dominates sample transport in particular devices, little discussion is provided to explain the difference in behavior and most reported rely on high EOF. The control of the net EOF through the device is an important parameter that is not held constant between the various devices. The net EOF through the system is limited by the nanofluidic element. 


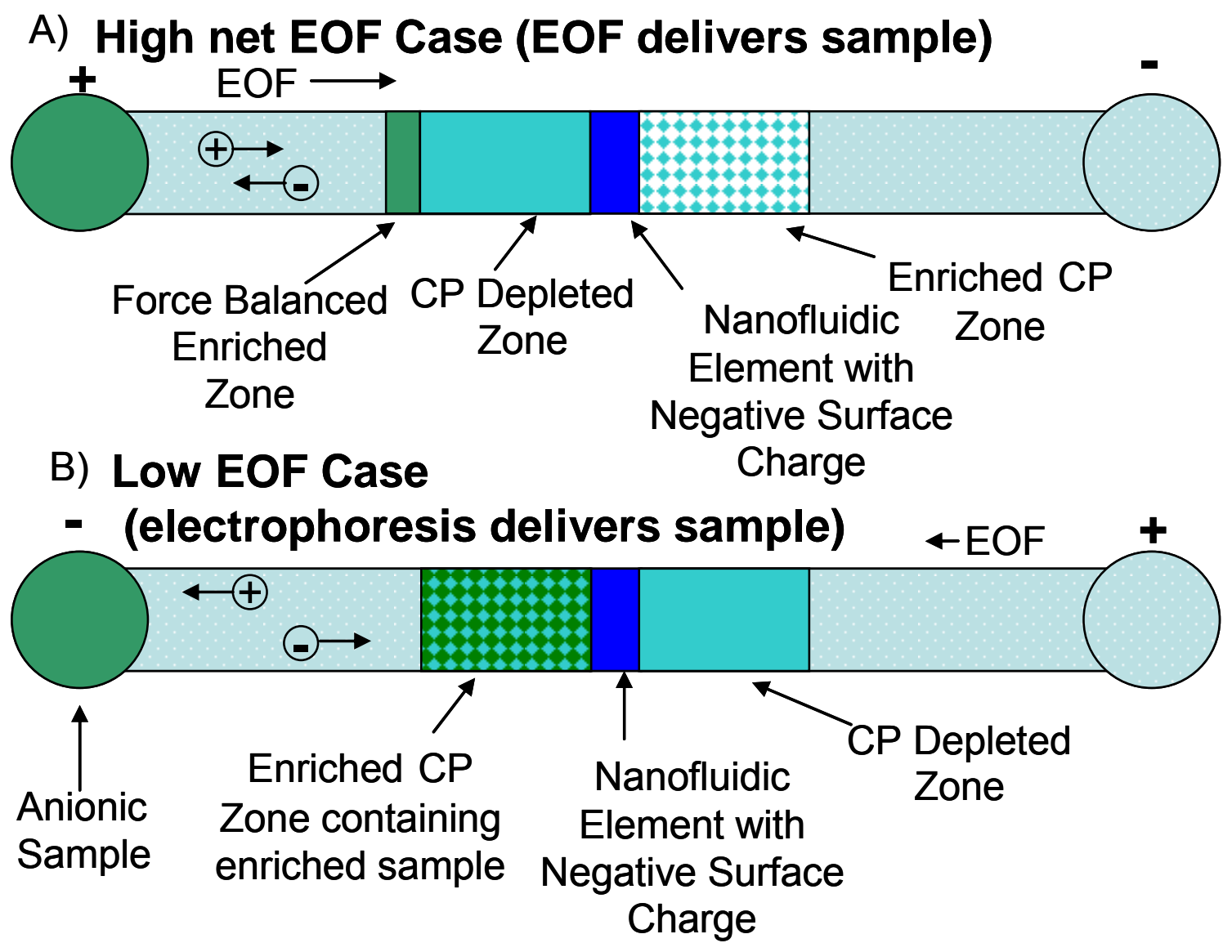

Figure 4.1: Schematic representation of the location of CP zones according to the applied voltage and location of enriched anionic sample in both high EOF and low EOF devices. In A), a schematic of CP developing in a high EOF device is shown. Three regions form: an enriched CP zone on the cathodic side of the nanofluidic element, a CP depleted zone on the anodic side of the nanofluidic element, and a third region called the force balanced enriched zone in which sample becomes enriched as a result of the balance between the forward EOF and reverse electrophoresis. Concentration polarization and high net EOF in the device are both required for this behavior to occur. Anionic samples are enriched in this region when a cation permselective nanofluidic element is used. In B), a schematic representation of sample becoming enriched in a low EOF device is shown. Two regions form: the enriched CP zone on the cathodic side of the nanofluidic element and the CP depleted zone on the anodic side of the nanofluidic element. The enriched CP and CP depleted zones reverse locations depending on the applied voltage. In the low EOF device, the sample becomes enriched in the enriched CP zone.

The devices that rely on electrophoresis for sample delivery have a variety of device characteristics. 2, 5, 9-11, 13, 19 Zhang et al. is the first device reported in the literature that behaves as an electrophoretic dominant concentrator. ${ }^{2}$ The 
device incorporates a nanocapillary membrane (NCM) into the fluidic design of a poly(dimethylsiloxane) (PDMS) device. Imaging of the concentration process near the NCM is challenging because the NCM is centered in a vertical channel. Other electrophoretic dominant concentrators incorporate gels or monoliths into fluidic designs fabricated in low EOF substrates. ${ }^{9-11,19}$ Pu et al. fabricated two microchannels in glass separated by a $100 \mu \mathrm{m}$ wide $\times 60 \mathrm{~nm}$ deep $\times 1 \mathrm{~mm}$ long nanochannel. While the nanochannel is large in comparison to other electrophoretic dominant concentrators, the highest buffer concentration at which CP was still observed was $1 \mathrm{mM} .^{5}$ Lastly, Foote et al. fabricated a device in glass that separated the two microchannels by a porous glass membrane. ${ }^{13}$ Therefore, several substrates have been used and various nanofluidic media have been incorporated into the devices that all result in electrophoretic transport of sample to become enriched.

Electrophoretic dominant concentrators are more amenable to proteomic samples than EOF dominant concentrators. The generation of EOF requires a substantial surface charge. It has been shown that proteins severely adsorb to charged surfaces leading to the damage of separations and sample loss. A proteomic sample concentrator must be compatible with coating techniques that minimize protein adsorption to surfaces. Two broad categories of coating techniques are dynamic and permanent coatings. Dynamic coatings interact with the surface through nonspecific adsorption and permanent coatings typically alter the surface through covalent linkages. ${ }^{28-32}$ Dynamic coatings require addition of electrolytes to the buffer, which is incompatible with downstream mass spectrometry that is vital to proteomics analyses. ${ }^{33}$ Another common method of minimizing protein adsorption is to alter the net surface charge to positive and use a low $\mathrm{pH}$ buffer. However, these techniques often reduce protein solubility ${ }^{34-}$ ${ }^{36}$ leading to clogging and sample loss. Neutral and hydrophilic polymer coatings have been shown to be the most successful permanent coatings at reducing protein adsorption, but simultaneously eliminate electroosmotic flow (EOF) by creating a neutral surface. ${ }^{33,37-41}$ Therefore, concentrators that rely on EOF for 
the delivery of the sample are not practical for the analysis of real world proteomic samples.

Concentrators that rely on electrophoresis for sample delivery are more desirable because they are generally more amenable to proteomic samples. Furthermore, concentrators that are capable of rapidly delivering and concentrating sample are also desirable. Electrophoretic dominant concentrators that show current rectification properties have added promise for real world proteomic concentrators. This potential stems from the ability of the current rectification process to produce higher currents in one direction than the other, similar to a diode. The higher current allows greater sample delivery because the resistance of the system is decreased.

Current rectification of microfluidic concentrators utilizing $\mathrm{CP}$ is a result of an asymmetric microfluidic design about a nanofluidic element. ${ }^{3,4}$ As CP develops under one polarity, the depleted zone is formed in the microchannel and under the opposite polarity the depleted zone is located in a macro-sized reservoir that has a much greater Reynold's number leading to an increase in mixing. Therefore, when the depletion zone is in the microchannel it remains intact and increases the resistance of the system causing a low current state, the OFF state. When the depletion zone is in the macro-sized reservoir, the depletion zone is disrupted leading to a lower resistance and allowing for a higher ON state current. Miller et al. was the first to report of a device that rectified current in the literature that was an EOF dominant concentrator. ${ }^{3,4}$ The concentration process of these devices occurs during the low OFF state current.

In this work, EOF and electrophoretic dominant concentrators are distinguished and two electrophoretic dominant designs are presented. An initial device is presented in which the sample delivery for the concentration process is electrophoresis and does not incorporate current rectification. However, the device exhibits poor concentration factors. Then, an asymmetric fluidic design that uses electrophoresis for sample delivery is implemented to create current rectification with a high ON state current as the sample is concentrating is presented. The current rectification leads to improved concentration factors of 
approximately 50 in about ten minutes with an improvement of $\sim 7$ times over the initial device. It is anticipated that the concentration factors will be further improved in future device designs.

\subsection{Experimental}

\subsubsection{Chemicals}

The sodium phosphate and fluorescein sodium salt were purchased from Sigma Aldrich (Milwaukee, WI). Sodium hydroxide, potassium chloride, sulfuric acid, hydrogen peroxide, and hydrofluoric acid were purchased from Fisher Scientific (Pittsburgh, PA). Chromium etchant was purchased from Transene (Danvers, MA). The developer used was MIF 315 and was purchased from Clariant (Somerville, NJ).

\subsubsection{Fabrication of Glass Devices}

The devices were fabricated out of chrome and AZ1500 photoresist coated D263 glass purchased from Telic (Valencia, CA). The pattern etched into the glass is shown in Figure 4.2 A) and was performed in such a manner that the distance separating the microfluidic features was approximately $15 \mu \mathrm{m}$. The pattern was produced using standard photolithography and wet chemical etching techniques. ${ }^{42}$ At the end of each microfluidic channel, a $360 \mu \mathrm{m}$ hole was drilled with drill bits from Kyocera Tycom Corporation (Irvine, CA) to allow fluidic connection to the microfluidic channel. Following drilling, the patterned wafer and a blank wafer were cleaned in an $85^{\circ} \mathrm{C}$ bath of piranha solution ( $3: 1$ solution of conc. sulfuric acid and $30 \%$ hydrogen peroxide, respectively). Each wafer was then high pressure cleaned in a wafer washer (model SWC111, Ultra $t$ Equipment Company, Fremont, $\mathrm{CA}$ ) and the wafers were pressed together to low temperature bond them. Finally, the wafers were high temperature bonded using a furnace (model 750-14, Fisher Scientific). ${ }^{42}$ Nanoports from Upchurch Scientific (Oak Harbor, WA) were attached to use as buffer reservoirs. 


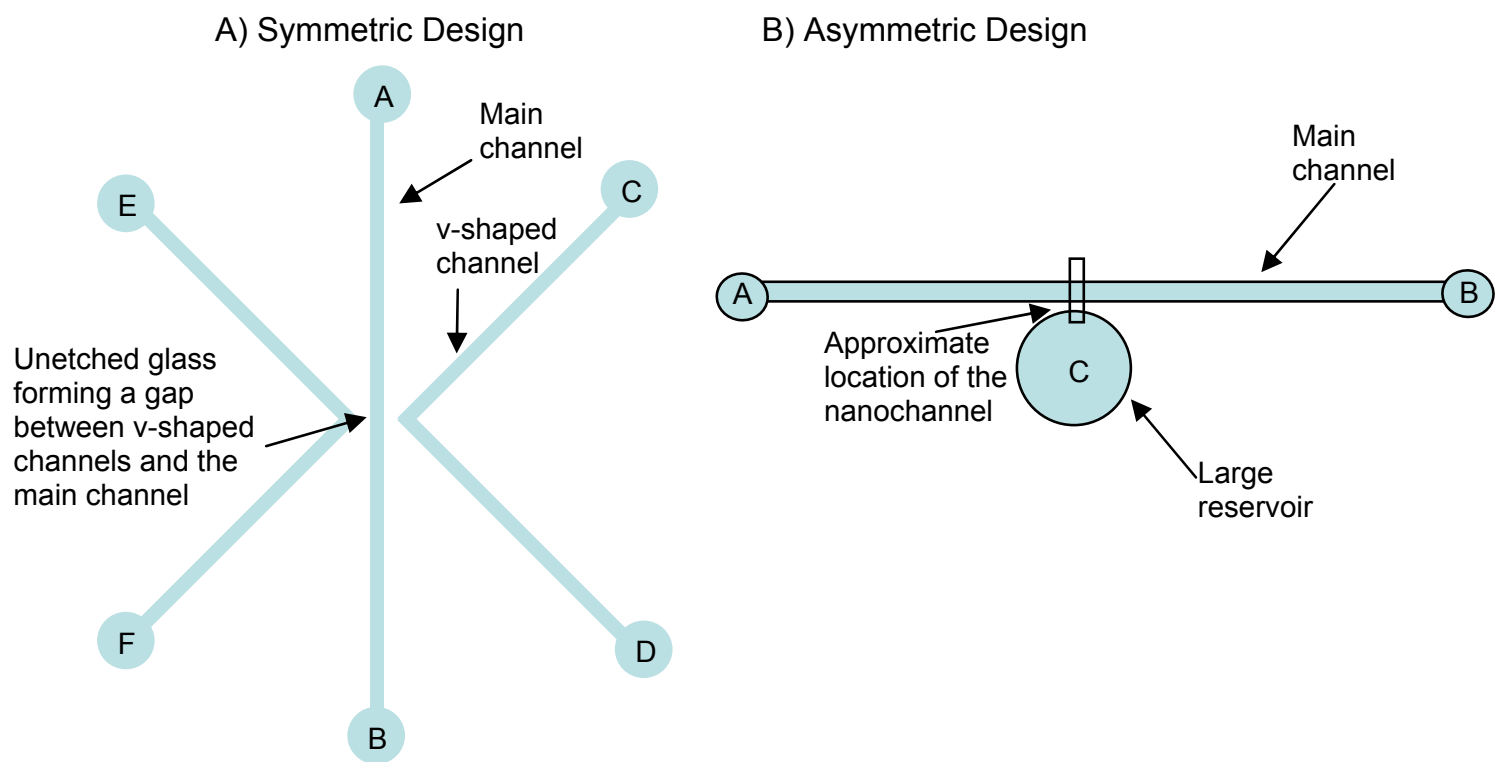

Figure 4.2: Schematics of device designs A) Schematic of symmetric glass microfluidic device. The channels are approximately $200 \mu \mathrm{m}$ wide and $20 \mu \mathrm{m}$ deep. The length of the channel connecting reservoirs $A \& B$ is $3 \mathrm{~cm}$ and the length of channels connecting reservoirs $C \& D$ and $E \& F$ is $3 \mathrm{~cm}$. The $v$-shaped channel is located $1.5 \mathrm{~cm}$ away from reservoir $A$. The gap between the v-shaped channels and the main channel is $\sim 15 \mu \mathrm{m}$. Note only one v-shaped channel is used. B) Schematic of asymmetric PDMS-glass hybrid microfluidic device. The main channel is $4 \mathrm{~cm}$ long and $200 \mu \mathrm{m}$ wide and $20 \mu \mathrm{m}$ deep and the large hole is $3 / 8$ " in diameter and equidistant from reservoirs A \& B. The gap between the microchannel and large hole is $100 \mu \mathrm{m}$. The nanochannel is aligned with the gap between the microchannel and the large reservoir and etched away following the bonding process..

\subsubsection{Formation and Measurement of the Nanochannel}

The $\sim 15 \mu \mathrm{m}$ thin glass wall between the two microfluidic channels allowed for the formation of a nanochannel between the two microfluidic channels. The nanochannel was created by first filling the microchannels with $20 \mathrm{mM}$ ammonium acetate. Then, electrical connections were made to reservoirs $B$ \& $D$ or $\mathrm{F}$ as shown in Figure 4.2 A). Ground was placed in reservoir $\mathrm{D}$ or $\mathrm{F}$ and the high voltage electrode was placed in reservoir $B$ along the main channel. A Matsusada power supply (HEOPS-10B2, Shiga, Japan) was the source of the high voltage and a Danbridge Megohmmeter (DB604, Roskilde, Denmark) was the grounding electrode. The high voltage started from zero and was slowly increased and a nanochannel typically formed at a voltage between 5 and $7 \mathrm{kV}$ 
due to dielectric breakdown of the glass. ${ }^{6,8}$ The current was monitored during the process and as the nanochannel formed a large spike from the nanoamp range to the microamp range was observed. Once the formation of the nanochannel was observed, the voltage was quickly turned off to ensure the nanochannel did not become too large.

The depth of the nanochannel was estimated using Equation 4.1 and by taking current measurements similar to Lee et al, as shown in Figure 4.3. ${ }^{8} \mathrm{~A}$ microscope image such as in Figure 4.3 A) was used to measure the length and width of the nanochannel and these values were used to calculate the nanochannel depth along with the following current measurements. Two different current measurements were performed by applying 1 to $8 \mathrm{~V}$ in increments of $1 \mathrm{~V}$ with a Leader power supply (Model: 718-3D, Cyprus, CA) along the $v$-shaped channel (i.e. reservoir $C \& D$ ) and across the nanochannel (between reservoir $B \& D$ ) while the device was filled with $100 \mathrm{mM}$ potassium chloride. The high concentration of salt was used to ensure concentration polarization did not develop within the microchannels and cause variations in the resistance of the system. The Megohmmeter was used as the grounding electrode in reservoir $B$ and allowed for the measurement of the currents that were in the nanoampere range. 
A)

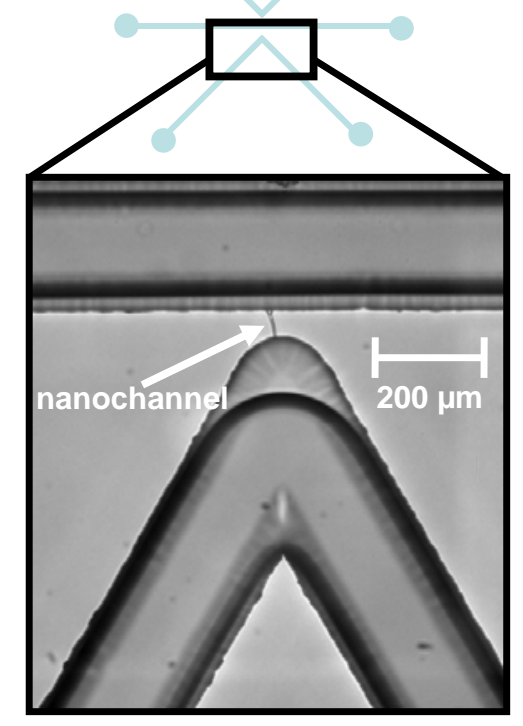

B)

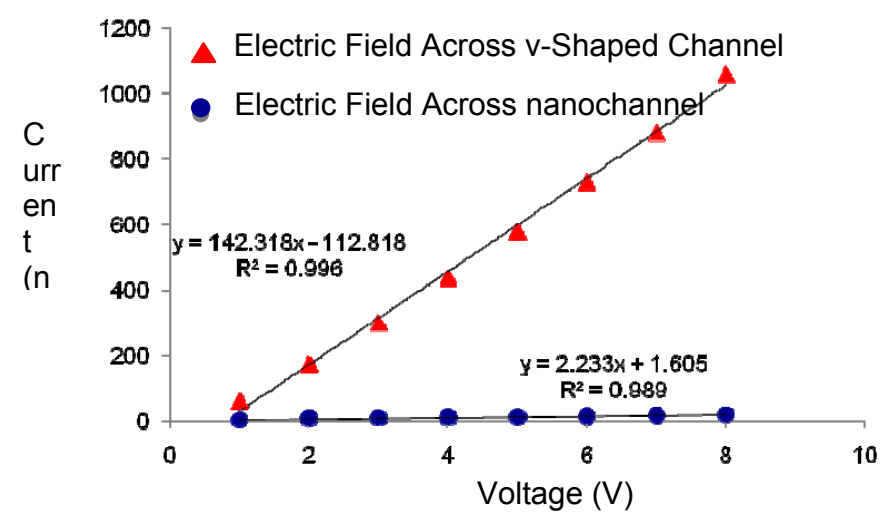

Figure 4 3: A) A representative micrograph acquired with a 10x objective of the microchannels and the nanochannel formed using dielectric breakdown. The micrograph was used to measure the length and width of the nanochannel that are $20 \mu \mathrm{m}$ and $4 \mu \mathrm{m}$, respectively. B) Current vs. voltage data collected to estimate the depth of the representative nanochannel. The data series in red represents measurement made across the v-shaped channel with electrodes placed in reservoirs $C \& D$ or $E \& F$. The data series in blue represents measurements acquired with an electrode placed in reservoir $B$ and the other electrode placed in reservoir $D$ or $F$. The resistance from the microchannels can be calculated from I-V curve (red series) and subtracted from I-V curve including the resistance of the nanochannel (blue series). The resistance of the nanochannel can be related to its dimensions ( $9.5 \mu \mathrm{m}$ long and $10.3 \mu \mathrm{m}$ wide) and with the only unknown dimension being the depth it can be calculated using Equation 4.1. The depth of this nanochannel is $\sim 19 \mathrm{~nm}$.

\subsubsection{Fabrication of Hybrid PDMS-Glass Devices}

A layer of chromium was patterned onto a glass slide to define the nanochannel. Corning glass slides (Fisher Scientific) with dimensions of 1" $x 3$ " and $\sim 1 \mathrm{~mm}$ thick were first cleaned in a $25 \%$ hydrogen peroxide to $75 \%$ sulfuric acid solution at $85^{\circ} \mathrm{C}$ for 45 minutes. Following cleaning, each glass slide was rinsed with deionized water produced from a Barnstead International Nanopure Infinity system (Dubuque, IA) with a nominal resistivity of $18.2 \mathrm{M} \Omega * \mathrm{~cm}$ and dried with nitrogen. Next, the slide was spin coated with hexamethyldisilazane 
(MichroChem, Newton, MA) at a rate of $4000 \mathrm{rpm}$ for 1 minute. The slide was then spin coated with AZ5214E photoresist (MichroChem, Newton, MA) for 1 minute at a rate of $5000 \mathrm{rpm}$. The slide was then baked for $55 \mathrm{~s}$ at $95^{\circ} \mathrm{C}$. A mask from Photoplot store (Colorado Springs, CO) containing a $200 \mu \mathrm{m}$ wide and $1 \mathrm{~mm}$ long channel was secured on the photoresist coated slide with a quartz wafer. The wafers were then exposed under a $365 \mathrm{~nm}$ UV lamp model 0130040-03 (Optical Association Inc San, Jose, CA) for $13 \mathrm{~s}$. The exposure resulted in the damaging of bonds in regions that were exposed to the UV light by the mask. The wafers were then developed in AZ MIF 300 developer for about $20 \mathrm{~s}$, dissolving the photoresist in the regions exposed to UV light. The wafers were rinsed with water and blown dry with nitrogen. The wafers were baked on a hotplate at $95{ }^{\circ} \mathrm{C}$ for 3 minutes. The wafers were then loaded into an E-beam evaporator model BJD-2000 (Temescal Livermore, CA) for chrome deposition and the dome rotation speed was set to 1-2 rpm. A $10 \mathrm{~nm}$ layer of chrome was deposited onto the glass wafers. The wafers were placed in acetone in a covered Petri dish overnight to carry out the lift off process. The wafers were then rinsed with acetone and blown dry with air. The final glass wafer had a single chrome feature deposited on it as illustrated in Figure 4.2 B).

To form a PDMS substrate that contained a microchannel and macroscopic reservoir, an acrylic mold was made by the in-house machine shop. It consisted of a raised $4 \mathrm{~cm}$ long and $200 \mu \mathrm{m}$ wide channel spaced $100 \mu \mathrm{m}$ from a 3/8" aluminum post as shown in Figure 4.2 B). To form the PDMS, Sylgard 184 silicone elastomer was weighed out with Sylgard 184 silicone elastomer curing agent (Dow Corning, Midland, MI) in a 10:1 ratio and mixed for 20 minutes on a stir plate. The PDMS mixture was then placed in a vacuum desiccator for 20 minutes to remove the bubbles. The PDMS was poured over the mold and placed back in the vacuum desiccator for an additional 20 minutes to remove any bubbles generated from the pouring process. The PDMS was placed in the oven for about 2 hours at $80^{\circ} \mathrm{C}$ to cure. Once the PDMS was cured, it was removed from the mold and access holes were punched in the PDMS using a hole punch with a punch size of 1/8". The PDMS was rinsed with isopropanol and dried with 
air and placed in a plasma cleaner model PDC-32G (Harrick Plasma, Ithaca, NY) along with a glass wafer with a chrome nanochannel. The plasma cleaner was set on high and left on for one minute. The PDMS and glass were removed from the plasma cleaner and aligned such that the chrome nanochannel spanned the $100 \mu \mathrm{m}$ gap between the 3/8" hole and the microchannel shown in Figure 4.2 B). The device was placed in an $80^{\circ} \mathrm{C}$ oven with weights evenly distributed on the device and left for about 2 hours to make a permanent bond between the glass and PDMS.

After the device was completely assembled, the chrome layer was used as a sacrificial layer. The microchannel and large hole were filled with chrome etchant and a few volts were applied across the nanochannel to aid in the etching process. Once the chrome was etched away, a nanosized channel remained. The dimensions of the nanochannel were measured using the method developed by Lee et al. and described previously in this work for the glass device. $^{8}$

\subsubsection{Enrichment Process and Epifluorescence Imaging}

The imaging of the glass devices was carried out using a Nikon TE-2000U (Melville, NY) inverted fluorescence microscope with a $4 x$ objective and Roper Scientific (Tucson, AZ) charge-coupled device model 650. Images were processed with MetaMorph software v6.2r6. A $10 \mathrm{mM}$ sodium phosphate buffer $\mathrm{pH} 7$ was loaded in the main channel and the buffer contained $20 \mu \mathrm{M}$ fluorescein was loaded in the v-shaped channel. The voltages applied (as indicated in Figure $4.4 \& 4.5$ ) during the concentration process were supplied and controlled by a Labsmith high voltage sequencer (model HVS448 6000D, Livermore, CA).

The imaging of the PDMS-glass hybrid devices was carried out using an Olympus IX 81 inverted fluorescence microscope (Center Valley, PA) with a Hamamatsu EM-CCD digital camera (model C9100-12, Bridgewater, NJ). The data was collected and processed using Slidebook v 4.1 (Denver, CO). A $10 \mathrm{mM}$ sodium phosphate buffer $\mathrm{pH} 7$ was used as the running buffer in the large macroscopic reservoir. The main channel was also loaded with running buffer, 
but the sample solution contained $20 \mu \mathrm{M}$ fluorescein in running buffer was placed in reservoirs A \& B. The voltages applied (as indicated in Figure 4.7 \& 4.9) during the enrichment process were also supplied and controlled by a Labsmith high voltage sequencer (model HVS448 6000D).

\subsubsection{Current Measurements}

To measure the current during the enrichment process, a $10 \mathrm{k} \Omega$ resistor was placed in series with the device and the voltage drop across the resistor was measured using a PC-interfaced digital multimeter (RadioShack, Fort Worth, TX) and Meterview version 1.0 recorded the data. The voltage drop could then be converted to current using Ohm's law. For the I-V curves presented in Figure 4.5, a program written in Labwindows 8.0 from National Instruments controlled the output of a TREK high voltage power supply (610E Medina, NY). The program applied $-800,-700,-600,-500,-400,-300,-200,-150,-100,-50,-25$, $10,0,10,25,50,100,150,200,300,400,500,600,700$, and 800 volts for five minutes each. The current was measured using the method described.

\subsection{Results and Discussion}

\subsubsection{Measurement of Nanochannel Height}

Before the devices were used, the nanochannel size is measured according to the method described in the experimental section. When the representative data presented in Figure $4.3 \mathrm{~B}$ ) is used to calculate the depth of the nanochannel, the nanochannel depth is found to be $\sim 19$ nanometers. The Equation 4.1 is used to calculate the depth of the nanochannel from the data presented. $^{8}$

$$
D_{\text {est }}=\rho \frac{L_{n}}{W_{n}}\left(\frac{1}{r_{B \text { to D }}}-\frac{1}{r_{C \text { to D }}}\right)^{-1}
$$

$D_{\text {est }}$ represents the estimated depth of the nanochannel, $\rho$ is the resistance of the electrolyte and in this case is $0.83 \Omega \bullet m, L_{n}$ is the length of the nanochannel, $W_{n}$ 
is the width of the nanochannel, $r_{B}$ to $D$ is the resistance from reservoir $B$ to $D, r_{C}$ to $D$ is the resistance from reservoir $C$ to $D$ (the slopes of the curves in Figure 4.3 B) can be used to calculate $r_{B}$ to $D$ and $r_{C}$ to $D$ ). The nanochannel is found to be $19 \mathrm{~nm}$ deep by $10.3 \mu \mathrm{m}$ wide by $9.3 \mu \mathrm{m}$ long. It should be noted that the channel is assumed to have a rectangular cross-section with this method.

Using the measured value of the nanochannel, the percentage of the nanochannel occupied by the double layer is estimated. According to the GuoyChapman theory of the double layer, ${ }^{43}$ the thickness of the double layer at the buffer concentrations used in the sample enrichment experiments $(10 \mathrm{mM})$ are 2 $\mathrm{nm}$ (assuming all singly charged ions) or $1 \mathrm{~nm}$ (assuming doubly charge ions). Using these calculations, the double layer occupies as much as $21 \%$ or as little as $11 \%$ of the nanochannel. Also the double layer thickness is defined as $1 / \mathrm{e}$, which is a rather arbitrary definition. Therefore, solution does not resemble the concentrations at bulk at defined double layer thickness. While the estimates of the double layer indicate that the nanochannel does not have double layer overlap, it has been suggested previously that $\mathrm{CP}$ is a positive feedback process in NMIs. ${ }^{44}$ Therefore, it is still possible the device will develop CP.

Once the depth of the nanochannel is estimated, the mode of sample delivery and an investigation into whether the device develops CP under the conditions used is performed. It should be noted that $10 \mathrm{mM}$ buffer concentration was chosen, because in capillary electrphoresis 10 to $50 \mathrm{mM}$ buffer concentrations are typically used while concentrations below $10 \mathrm{mM}$ increase the length of the Debye layer. In Figure 4.4, the behavior of fluorescein in the symmetric device is observed. In image Figure $4.4 \mathrm{~A}$ ), no voltages are applied and the v-shaped channel has been filled with buffer containing fluorescein while the main channel has only been filled with buffer. The intensity of the fluorescein is not uniform throughout the v-shaped channel. The non-uniformity in the intensities may be caused from slightly different solution level heights between reservoirs $C \& D$ or maybe an uneven illumination from the microscope. However, it is important to note the fluorescein does not pass through the 
nanochannel into the main channel indicating that the nanochannel is cation permselective.

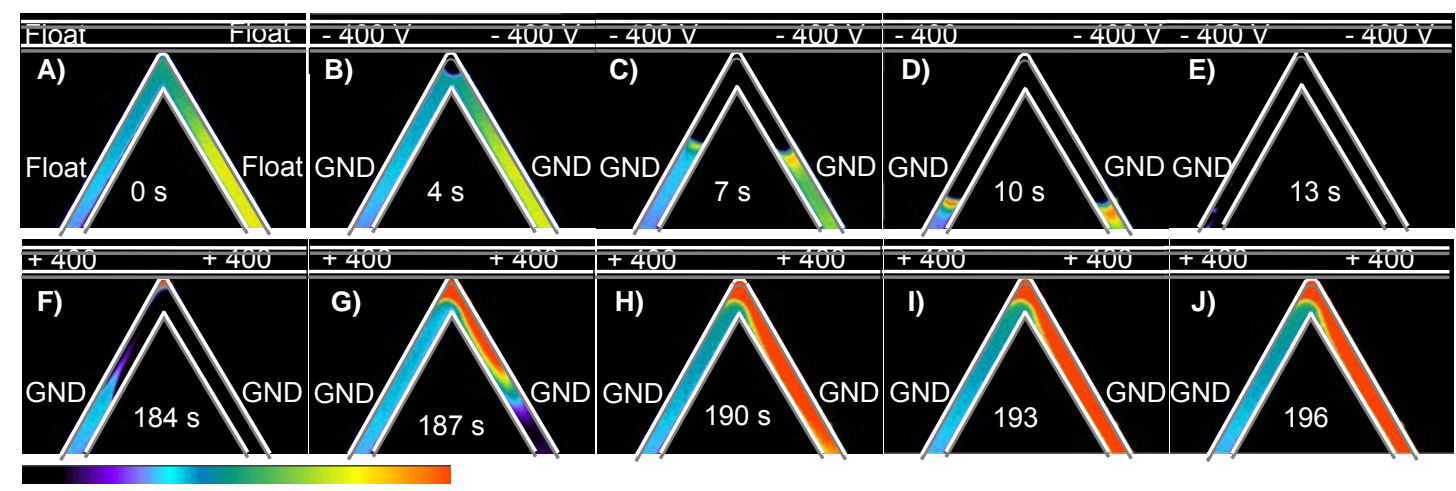

Figure 4.4: The symmetric device design is used to obtain the data presented and only one vshaped channel was utilized to acquire the data. The images are scaled in pseudocolor to aid in differentiating intensities throughout the channel. Images are acquired with the Nikon microscope using the $4 x$ objective and a $150 \mathrm{~ms}$ exposure time. The $v$-shaped channel is filled with $10 \mathrm{mM}$ sodium phosphate at $\mathrm{pH} 7$ buffer containing $20 \mu \mathrm{M}$ fluorescein and the main channel is filled with buffer. In image a, all of the electrodes are floating. In images B-E, ground is applied along the $\mathrm{V}$-shaped channel and $-400 \mathrm{~V}$ along the main channel. In images F-J the polarities are reversed, ground is applied on the $v$-shaped channel and $+400 \mathrm{~V}$ along the main channel.

In Figure 4.4 B), after a -400 V is applied to the main channel and the v-shaped channel is grounded, the fluorescein quickly migrates away from the nanochannel and a region develops that has an intensity comparable to the background. This region of low intensity is the ion depletion zone and develops under the expected applied voltage. The cations (counter-ions) are able to migrate through the nanochannel with a negative surface charge, but the anions are restricted. As the cations leave the solution at the nanochannel/microchannel interface as shown in Figure 4.1 B), the anions are forced to migrate away from the interface to uphold electroneutrality forming the depleted CP zone. Over time, the depleted CP zone becomes larger and actually fills the whole field of view (Figures 4.4 C-E). Interestingly, the fluorescein does appear to become enriched at the edge of this ion depletion zone initially, presumably because the EOF is low. 
As the polarity is reversed (Figure 4.4 F), the fluorescein migrates rapidly to the nanochannel with a large intensity and indicates that the enriched CP zone is now forming in the v-shaped channel. Despite EOF opposing the migration of fluorescein toward the $+400 \mathrm{~V}$ potential, it is still able to migrate toward the main channel as shown in Figures 4.4 F-J. The migration of the fluorescein, opposite EOF, indicates that the net EOF through the system must low. Typically, glass microfluidic devices create high EOF above $\mathrm{pH} 4$ and this experiment is at $\mathrm{pH} 7$, well above $\mathrm{pH} 4$ allowing for deprotonated silanol groups. The EOF of glass microfluidic devices routinely dominates the electrophoretic transport of fluorescein. Thus, the most logical explanation for the transport of fluorescein to occur according to electrophoresis is that the single nanochannel actually suppresses the bulk flow of EOF through the device due to extremely small cross-sectional area resulting in low net EOF through the nanochannel. The designed device can therefore be classified as an electrophoretic dominant concentrator, because electrophoresis is the mechanism for analyte transport that leads to enriched sample. As time passes, (Figure $4.4 \mathrm{G}-\mathrm{J}$ ) the length of the enriched fluorescein grows in the channel supporting the idea of an electrophoretic dominant concentrator. The right side of the v-shaped channel continues to display higher intensities than the left side of the v-shaped channel similar to Figure $4.4 \mathrm{~A}$ ) under all floating potentials.

The best concentration factor obtained with these symmetric glass devices is about 7 using $20 \mu \mathrm{M}$ as the starting concentration of fluorescein, which is poor

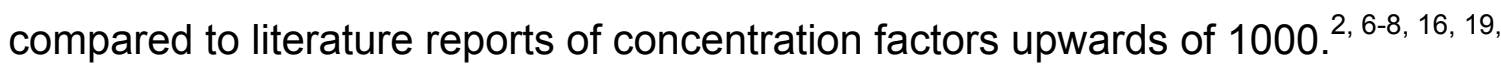
21, 24, 45 However, it has been shown in the literature that the concentration factor is dependent on the initial concentration of the analyte and lower initial concentration yields a greater concentration factor. ${ }^{7,8}$ Therefore, the concentration factors within this work could be improved by using lower initial concentrations. In Figure 4.5, the intensity vs. time is presented for the symmetric device. The concentration factor after $600 \mathrm{~s}$ is 4.5 , but the concentration is not stable over time and the best concentration factor for this experiment is 5.7 at $360 \mathrm{~s}$. 


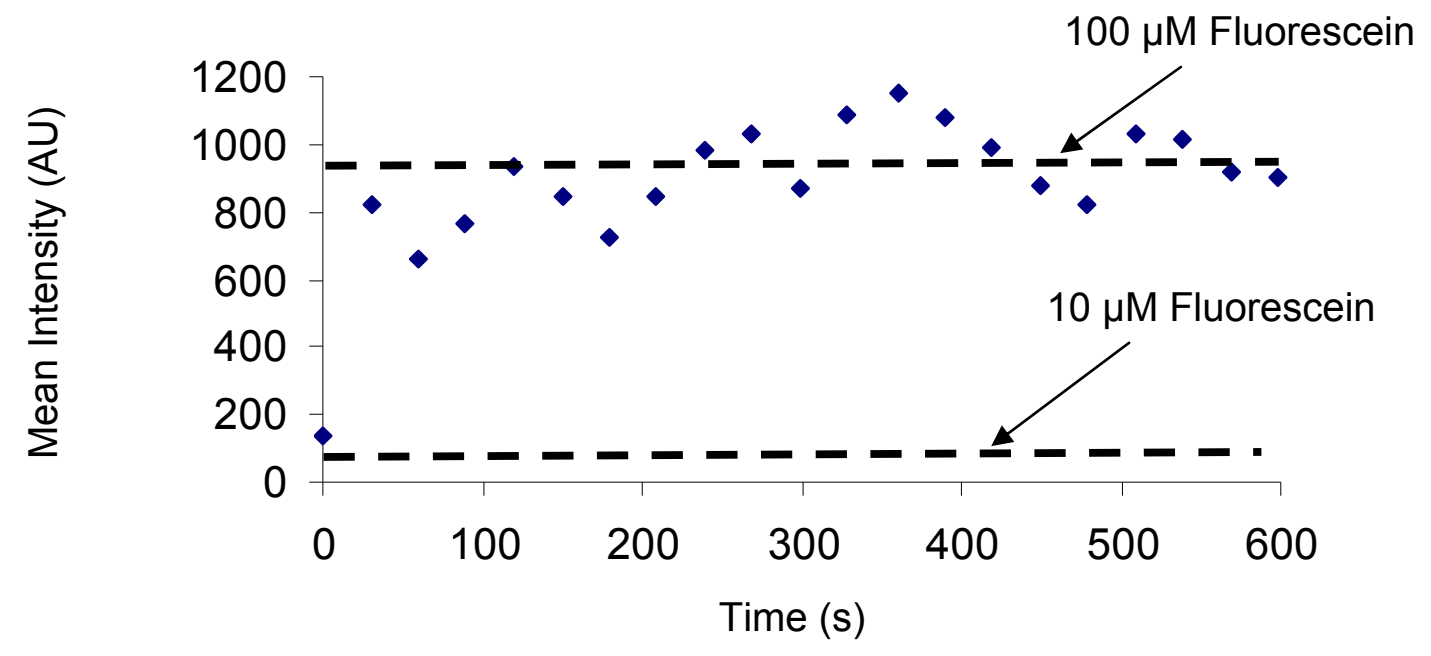

Figure 4.5: The average intensity vs. time is presented for the symmetric device. After $600 \mathrm{~s}$, the concentration is about $90 \mu \mathrm{M}$, which corresponds to a concentration factor of 4.5 . The concentration fluctuates with time and the highest concentration is observed at $360 \mathrm{~s}$. The concentration is about $114 \mu \mathrm{M}$ and corresponds to a concentration factor of 5.7. The intensities are extracted from images collected using the $4 x$ objective, 25 ms exposure time, $30 \mathrm{~s}$ image capture frequency. A positive $600 \mathrm{~V}$ is placed in the reservoirs $\mathrm{A} \& \mathrm{~B}$ and ground in reservoirs $\mathrm{C}$ $\& D$. The buffer is $10 \mathrm{mM}$ phosphate buffer at $\mathrm{pH} 7$ and the initial concentration of fluorescein is $20 \mu \mathrm{M}$. The nanochannel used to collect this data is $37 \mathrm{~nm}$ deep.

To determine the concentrator device did not rectify current as expected, the current is monitored with applied voltage. ${ }^{3}$ In Figure 4.6, the current vs. voltage plot displays an ohmic response regardless of the location of the applied high voltage. 


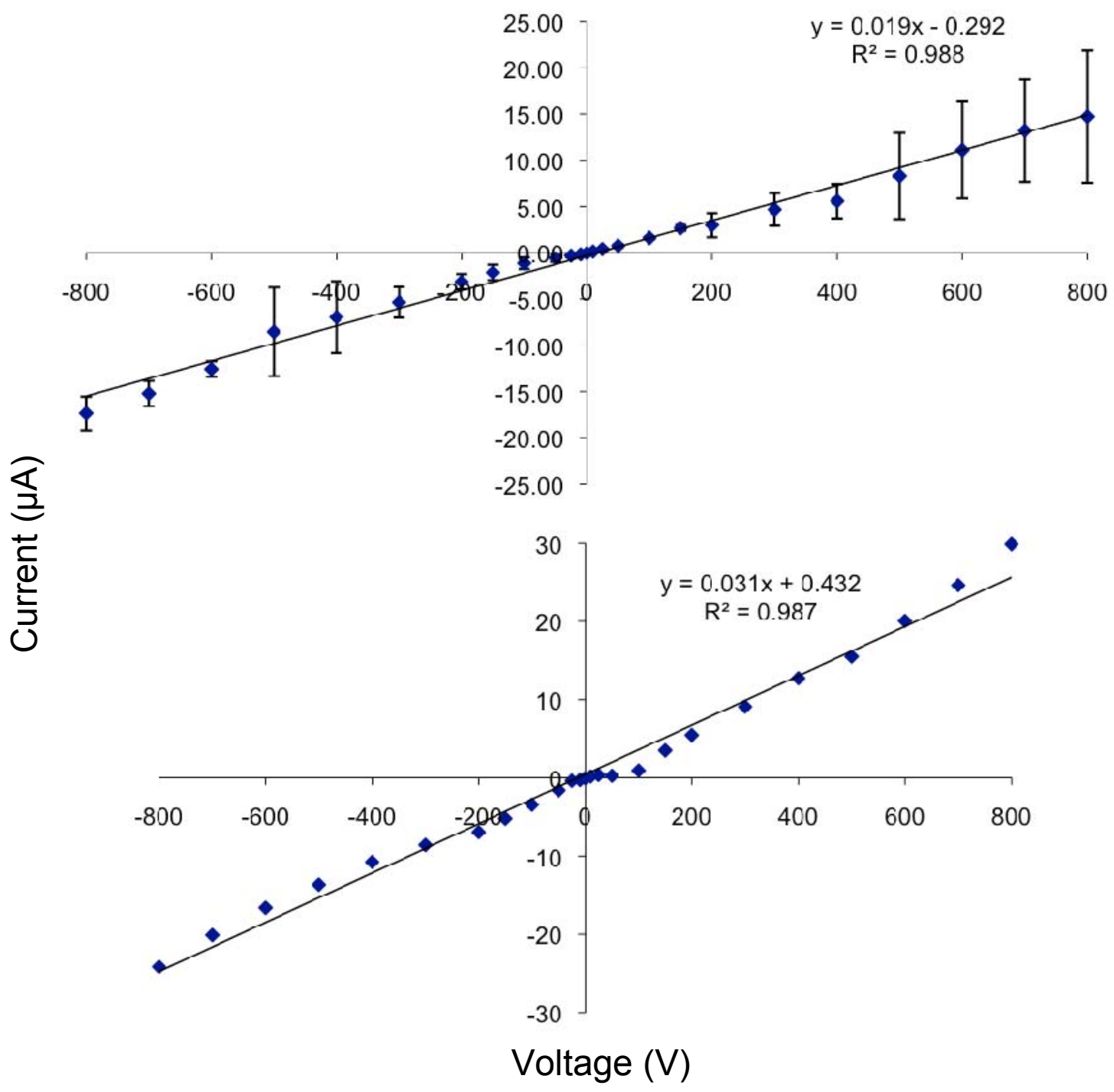

Figure 4.6: Current vs. voltage plots for the symmetric device. A) The high voltage was applied on the v-shaped channel and ground was held on main channel. The results indicate that the symmetric device does not rectify current as expected. B) The high voltage was applied on the main channel while ground was held on the v-shaped channel.

Other devices capable of enriching analyte have been reported to rectify ionic current. ${ }^{3,4}$ However, these devices that report non-ohmic responses have fluidic designs that are asymmetric about the nanofluidic element and the non-ohmic response has been attributed to the asymmetric fluidic design. The ohmic response observed with this design is expected, because regardless of the applied polarity one side of the nanochannel will have a CP depleted zone and the other side will have an enriched CP zone and both sides of the nanochannel 
are micro-sized allowing for the sustained existence of both CP zones simultaneously.

While the design does offer a concentrator compatible with a coating used to reduce protein surface adsorption that simultaneously minimizes EOF, the device falls short in providing an adequate concentration factor. As the analyte becomes enriched, the CP depleted zone is formed in the main channel and causes a high resistance throughout the whole system. This high resistance leads to low current through the device and subsequently low sample delivery. The low net EOF of this device could be combined with an asymmetric fluidic design to increase current during the enrichment process to increase speed and maximum concentration factor achievable. Therefore, a large outlet reservoir is incorporated into a different device design to provide current rectification as shown in Figure 4.2 B). To facilitate easier device fabrication a hybrid PDMS/glass device is created where micro-sized and macro-sized features were molded into the PDMS and bonded to a glass wafer containing the $10 \mathrm{~nm}$ thick layer of deposited chrome. The micro-sized and macro-sized feature must be fabricated with a small distance between them, so it becomes challenging to fabricate the micro-sized and macro-sized feature.

After the chrome is etched away, the depth of the nanochannel of the PDMS-glass hybrid device is measured according to the method described above. The nanochannel measures as $\sim 23 \mathrm{~nm}$ deep. After nanochannel measurement, a study is performed to determine the mechanism of sample transport and if CP develops in this device as observed in Figure 4.7 A) \& B). In the first row of images, $+100 \mathrm{~V}$ are applied to the large reservoir and ground to the main channel with fluorescein located in reservoirs $A \& B$ and buffer in reservoir $C$. The first image of panel $A$ ) shows the arrival of fluorescein from the left side and the fluorescein already appears to be slightly enriched when compared to a $20 \mu \mathrm{M}$ standard of fluorescein under the same imaging conditions. The fluorescein takes a long time to migrate to the interface of the nanochannel and the microchannel. The second image of panel A) shows fluorescein 
continuing to migrate toward the center of the main channel, but again the enriched fluorescein is not symmetric about the nanochannel.

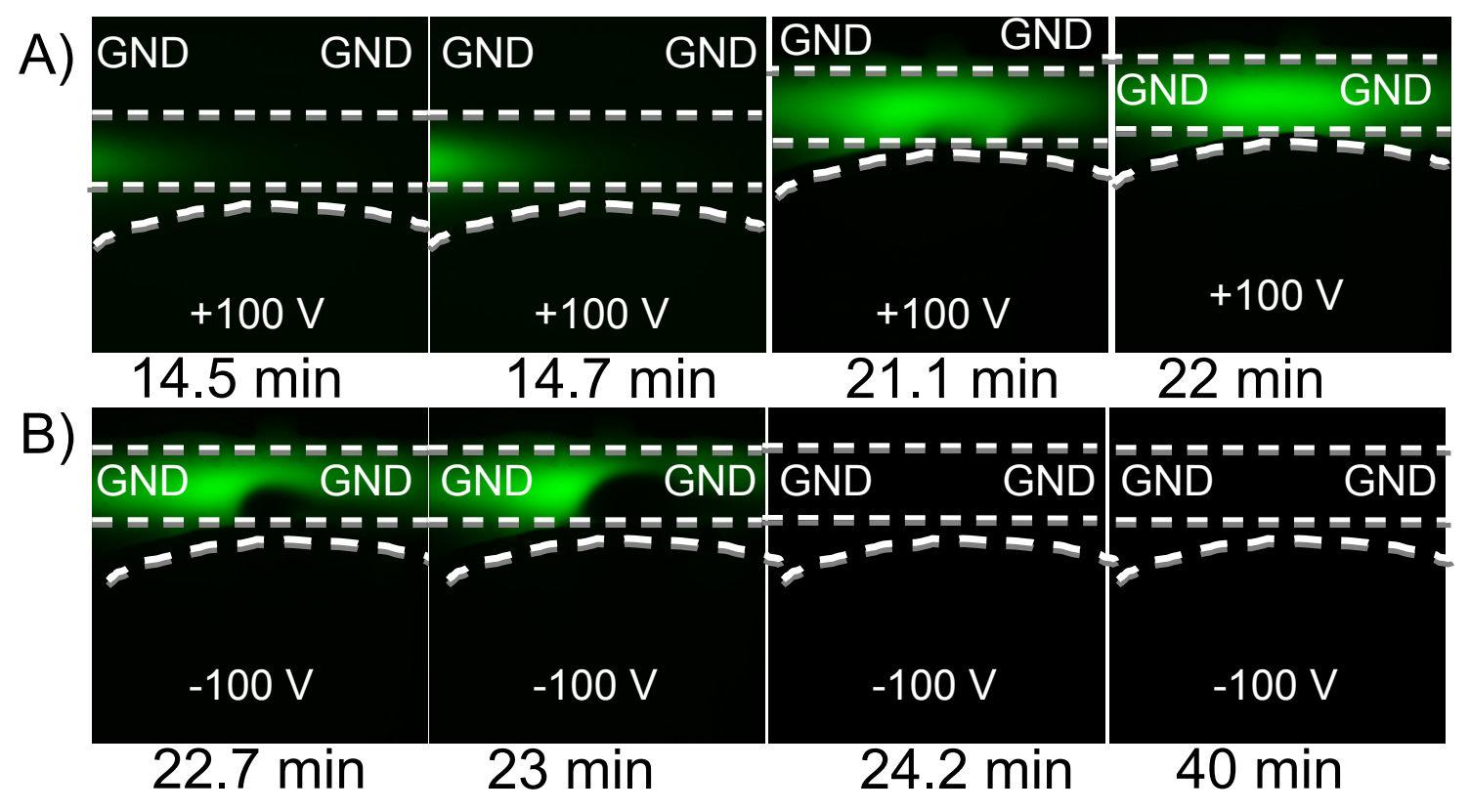

Figure 4.7: Asymmetric device is used to collect the data presented. In row A) of images, the applied voltages cause sample enrichment in the microchannel. At the start of the experiment, the microchannel and large reservoir contain buffer without fluorescein and buffer containing fluorescein is placed in reservoirs A \& B. Initially, it takes a long time for the sample to reach the NMI, but once it does it rapidly increases in concentration. The first two frames use a 10x objective, and a $25 \%$ neutral density filter, and a $100 \mathrm{~ms}$ exposure time in A). The remaining frames in A) and B) still use a 10x objective, but a $6 \%$ neutral density filter and a $25 \mathrm{~ms}$ exposure time are used instead. In row $B$ ) of images, the voltages are applied such that the device has a depletion zone in the microchannel. B) Current vs. time plot that corresponds to the fluorescence imaging for the asymmetric device. The $\mathrm{ON}$ state corresponds to ground applied to the main channel and $+100 \mathrm{~V}$ applied to the large reservoir while the OFF state corresponds to $+100 \mathrm{~V}$ applied to the main channel and ground to the large reservoir. Note the current slowly decays in the OFF state.

The third frame of panel A) shows the main channel beginning to fill with fluorescein and finally in the fourth frame of panel A) the main channel is brightly filled with fluorescein. It is important to note that from the third and fourth frames of panel A) have a different set of imaging settings than frames 1 and 2 of panel A), because the fluorescein became so enriched the camera was saturating. 
Frames 1 and 2 in panel A) are acquired with a neutral density filter that allows $25 \%$ of the excitation light to pass and an exposure time of $100 \mathrm{~ms}$ while frames 3-4 of panel A) and frames 1-4 of panel B) use a neutral density filter that only allows $6 \%$ of the excitation light to pass and a $25 \mathrm{~ms}$ exposure time. Therefore, intensities may not appear much increased in comparison to frames 1 and 2 of panel A) however the amount of fluorescein has actually increased. As the polarity is switched to a negative applied voltage in the large reservoir and ground remains along the main channel, the depletion zone grows into the microchannel as expected. Again, the cations are able to pass through the nanochannel while the anions are unable to pass. The anions must migrate away from the nanochannel in order to maintain electroneutrality leading to the formation of the depletion zone in the microchannel. As time passes, the fluorescence in the main channel reduces to the level of the background. The PDMS-glass hybrid device behaves similarly to the glass device in that fluorescein migrates based on electrophoresis again indicating the net EOF through the system is low. The low net EOF through the system can be attributed to both the lower EOF produced from PDMS in comparison to glass as well as the nanochannel restricting the net EOF through the device and allowing fluorescein to be transported electrophoretically.

The behavior of the current through the hybrid device is reported in Figure 4.8. The current vs. time plot shows as $+100 \mathrm{~V}$ is applied to the large reservoir and ground is applied to the main channel (enrichment of fluorescein in the microchannel is occurring at these voltages) the average current is $\sim 9.2 \mu \mathrm{A}$. When the polarity is switched to negative, the average quasi-steady state current dramatically decreases to $\sim 1.4 \mu \mathrm{A}$. The device exhibits current rectification at $\pm 100 \mathrm{~V}$ as expected by the asymmetric fluidic design about the nanochannel. The ON state of the rectifier occurs during the enrichment process unlike other devices reported in the literature that enrich sample in the OFF state. ${ }^{3,4}$ The net EOF through the system appears to be a critical parameter that governs the device operation as shown in Figure 4.1. The rectification factor is about 6.6 times at $\pm 100 \mathrm{~V}$ and the literature reports a device with a rectification factor of 17 
times, however, this report was at $\pm 800 \mathrm{~V} .^{3}$ The high ON state current aids in the delivery of more sample to the microchannel-nanochannel interface during the concentration process. Thus, the first ionic current recitifying device that enriches sample during the ON state and delivers sample electrophoretically is reported.

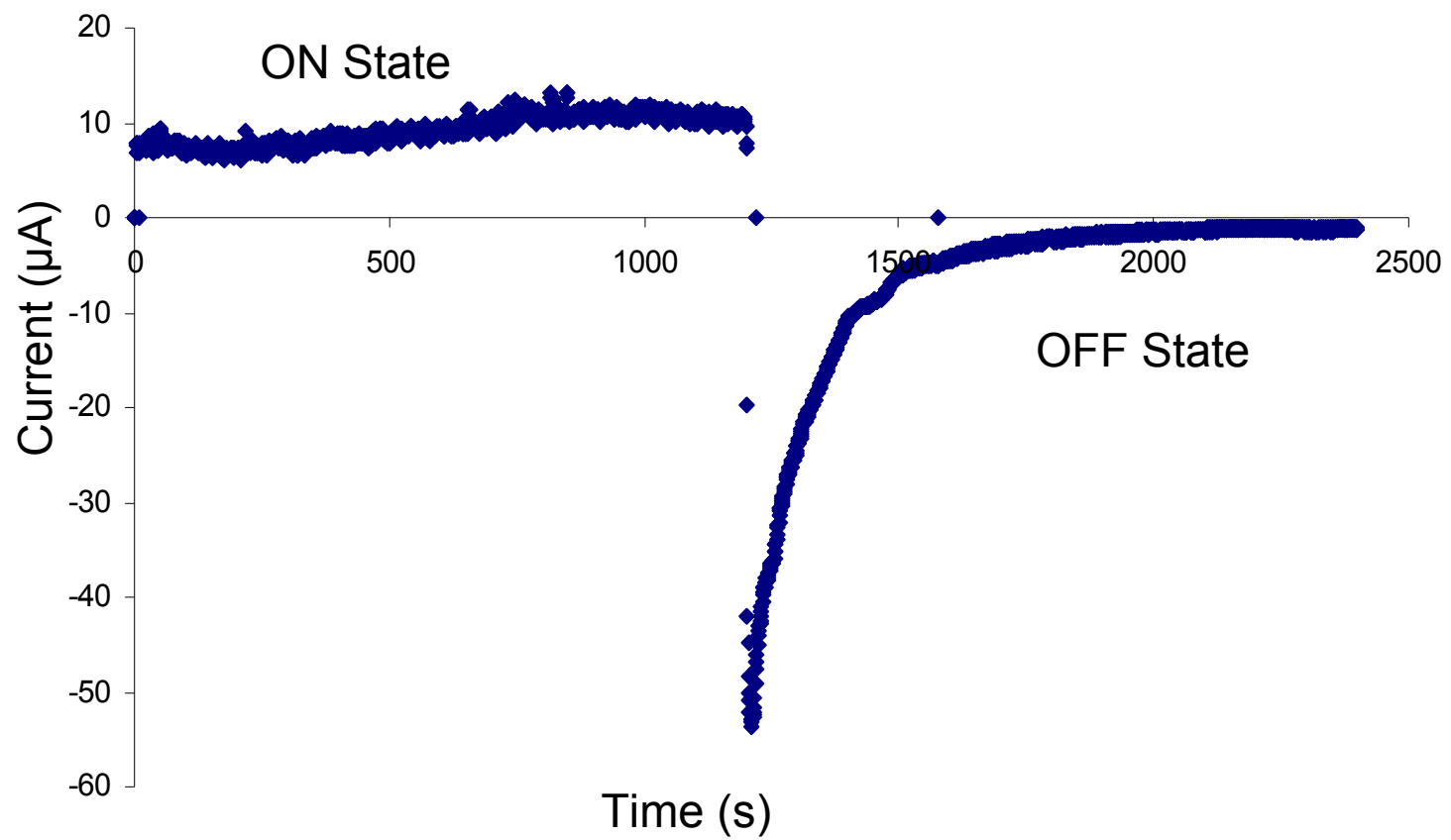

Figure 4.8: Current vs. time plot that corresponds to the fluorescence imaging in Figure 4.7. The ON state corresponds to ground applied to the main channel and $+100 \mathrm{~V}$ applied to the large reservoir while the OFF state corresponds to $+100 \mathrm{~V}$ applied to the main channel and ground to the large reservoir.

The best concentration factor obtained with the asymmetric PDMS-glass hybrid device is $\sim 60$. While this is still below several concentration factors reported in the literature, it is an improvement in comparison to the symmetric device reported previously in this chapter. In Figure 4.9, the intensity vs. time is presented for the asymmetric device. Similar stability problems are observed for the asymmetric design in which the intensity and thus the concentration increase but as time passes the intensity decreases again. The instability of devices that rely on $\mathrm{CP}$ has been reported in the literature. ${ }^{46}$ The instability of the devices is not the focus of this work and will require future work to improve the stability of 
these devices. The concentration factor is 50 at $600 \mathrm{~s}$ and at its best is 62 after only $305 \mathrm{~s}$. The asymmetric design shows improved concentration because of its ability to deliver sample more readily during ON state or high current conditions.

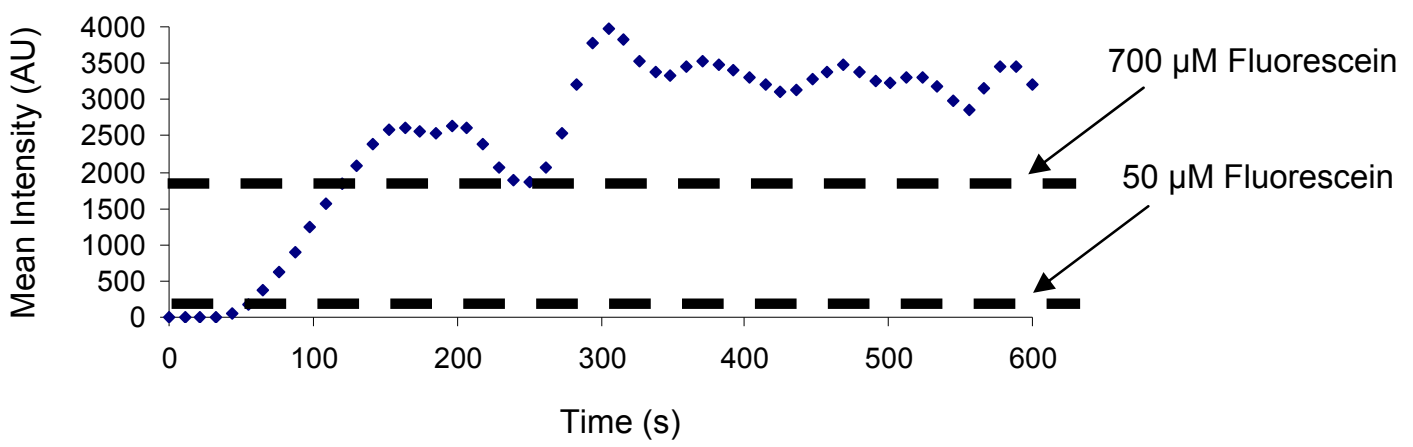

Figure 4.9: The average intensity vs. time is presented for the asymmetric device. After $600 \mathrm{~s}$, the concentration is about $1000 \mu \mathrm{M}$, which corresponds to a concentration factor of 50 . Again, the concentration fluctuates with time and the highest concentration is observed at $305 \mathrm{~s}$. The concentration is about $1235 \mu \mathrm{M}$ and corresponds to a concentration factor of 62 . The intensities are extracted from images collected using the 10x objective, $25 \mathrm{~ms}$ exposure time, $11 \mathrm{~s}$ image capture frequency. A positive $100 \mathrm{~V}$ is placed in the reservoir $\mathrm{C}$ and ground in reservoirs $\mathrm{A} \& \mathrm{~B}$. The buffer is $10 \mathrm{mM}$ phosphate buffer at pH 7 and the initial concentration of fluorescein is 20 $\mu \mathrm{M}$. The nanochannel used to collect this data is $23 \mathrm{~nm}$ deep.

\subsection{Conclusions}

In this work, two NMI concentrators are developed that have low net EOF and are therefore compatible with netural coatings, such as PEG silanes, that minimize non-specific protein adsorption. Initially a symmetric device fabricated of glass with a single nanochannel is investigated. The symmetric glass device delivers anionic sample electrophoretically and is compatible with coatings that reduce surface adsorption while simultaneously reducing EOF. The glass device is capable of concentrating anionic analytes, but the best concentration factor achieved is 7 . The symmetric fluidic design about the nanochannel of the glass device does not rectify current and is consistent with reports in the literature. A second device made of PDMS and glass is designed with an asymmetric fluidic design about the nanochannel to result in a PDMS-glass hybrid device that rectifies ionic current. The hybrid device is still compatible with coatings that 
reduce surface adsorption and EOF, but rectifies ionic current as well.

Concentration factors of at least 50 are obtained with the asymmetric device as it enriches sample during the ON state of the rectification leading to increased sample delivery. Enrichment is initially slow, but becomes faster and may be a result of the positive feedback associated with the depletion zone enhancing the permselectivity of the nanochannel. In the future, lower initial concentrations similar to other literature reports will be investigated to improve the concentration factors. Better fabrication methods to form the nanochannel are needed to improve the stability of the devices. Although some improvement is still needed a mobility dominant concentrator based on a nanochannel is realized and the current rectifying device increases current and the enrichment speed. More reliable nanochannel fabrication and higher currents will provide greater concentration factors and more rapid speeds. This work presents a new NMI analyte concentrator that uses low EOF and consequently is compatible with neutral coatings that minimize protein adsorption. 


\subsection{References}

(1) Ghaemmaghami, S.; Huh, W.-K.; Bower, K.; Howson, R. W.; Belle, A.; Dephoure, N.; O'Shea, E. K.; Weissman, J. S. Global analysis of protein expression in yeast Nature 2003, 425, 737-741.

(2) Zhang, Y.; Timperman, A. T. Integration of nanocapillary arrays into microfluidic devices for use as analyte concentrators Analyst (Cambridge, United Kingdom) 2003, 128, 537-542.

(3) Miller, S. A.; Kelly, K. C.; Timperman, A. T. Ionic current rectification at a nanofluidic/microfluidic interface with an asymmetric microfluidic system Lab on a Chip 2008, 8, 1729-1732.

(4) Kelly, K. C.; Miller, S. A.; Timperman, A. T. Investigation of Zone Migration in a Current Rectifying Nanofluidic/Microfluidic Analyte Concentrator Anal. Chem. 2009, 81, 732-738.

(5) Pu, Q.; Yun, J.; Temkin, H.; Liu, S. Ion-Enrichment and Ion-Depletion Effect of Nanochannel Structures Nano Lett 2004, 4, 1099-1103.

(6) Kim Sun, M.; Burns Mark, A.; Hasselbrink Ernest, F. Electrokinetic protein preconcentration using a simple glass/poly(dimethylsiloxane) microfluidic chip Anal Chem 2006, 78, 4779-4785.

(7) Wang, Y.-C.; Stevens, A. L.; Han, J. Million-fold Preconcentration of Proteins and Peptides by Nanofluidic Filter Anal Chem 2005, 77, 42934299.

(8) Lee, J. H.; Chung, S.; Kim, S. J.; Han, J. Poly(dimethylsiloxane)-Based Protein Preconcentration Using a Nanogap Generated by Junction Gap Breakdown Anal Chem 2007, 79, 6868-6873.

(9) Dhopeshwarkar, R.; Crooks Richard, M.; Hlushkou, D.; Tallarek, U. Transient effects on microchannel electrokinetic filtering with an ionpermselective membrane Anal Chem 2008, 80, 1039-1048.

(10) Dai, J.; Ito, T.; Sun, L.; Crooks, R. M. Electrokinetic trapping and concentration enrichment of DNA in a microfluidic channel Journal of the American Chemical Society 2003, 125, 13026-13027.

(11) Dhopeshwarkar, R.; Sun, L.; Crooks, R. M. Electrokinetic concentration enrichment within a microfluidic device using a hydrogel microplug $L a b$ on a Chip 2005, 5, 1148-1154.

(12) Khandurina, J.; Jacobson, S. C.; Waters, L. C.; Foote, R. S.; Ramsey, J. M. Microfabricated Porous Membrane Structure for Sample Concentration and Electrophoretic Analysis Anal Chem 1999, 71, 1815-1819.

(13) Foote, R. S.; Khandurina, J.; Jacobson, S. C.; Ramsey, J. M. Preconcentration of proteins on microfluidic devices using porous silica membranes AnalChem 2005, 77, 57-63.

(14) Hlushkou, D.; Dhopeshwarkar, R.; Crooks Richard, M.; Tallarek, U. The influence of membrane ion-permselectivity on electrokinetic concentration enrichment in membrane-based preconcentration units $L a b$ on a chip 2008, 8, 1153-1162.

(15) Huang, K.-D.; Yang, R.-J. A nanochannel-based concentrator utilizing the concentration polarization effect Electrophoresis 2008, 29, 4862-4870. 
(16) Yu, H.; Lu, Y.; Zhou, Y.-g.; Wang, F.-b.; He, F.-y.; Xia, X.-h. A simple, disposable microfluidic device for rapid protein concentration and purification via direct-printing Lab on a Chip 2008, 8, 1496-1501.

(17) Wang, S.-C.; Wei, H.-H.; Chen, H.-P.; Tsai, M.-H.; Yu, C.-C.; Chang, H.-C. Dynamic superconcentration at critical-point double-layer gates of conducting nanoporous granules due to asymmetric tangential fluxes Biomicrofluidics 2008, 2, 014102.

(18) Stein, D.; Deurvorst, Z.; Heyden, F. H. J. v. d.; Koopmans, W. J. A.; Gabel, A.; Dekker, C. Electrokinetic Concentration of DNA Polymers in Nanofluidic Channels Nano Lett 2010, DOI: 10.1021/nl902228p.

(19) Yamamoto, S.; Hirakawa, S.; Suzuki, S. In Situ Fabrication of lonic Polyacrylamide-Based Preconcentrator on a Simple Poly(methylmethacrylate) Microfluidic Chip for Capillary Anal. Chem. 2008, 80, 8224-8230.

(20) Lee, J. H.; Song, Y.-A.; Tannenbaum, S. R.; Han, J. Increase of Reaction Rate and Sensitivity of Low-Abundance Enzyme Assay Using Micro/Nanofluidic Preconcentration Chip Anal Chem 2008, 80, 3198-3204. (21) Hoeman, K. W.; Lange, J. J.; Roman, G. T.; Higgins, D. A.; Culbertson, C. $\mathrm{T}$. Electrokinetic trapping using titania nanoporous membranes fabricated using sol-gel chemistry on microfluidic devices Electrophoresis 2009, 30, 3160-3167.

(22) Zhou, K.; Kovarik, M. L.; Jacobson, S. C. Surface-Charge Induced Ion Depletion and Sample Stacking near Single Nanopores in Microfluidic Devices J. Am. Chem. Soc. 2008, 130, 8614-8616.

(23) Kovarik, M. L.; Jacobson, S. C. Integrated Nanopore/Microchannel Devices for ac Electrokinetic Trapping of Particles Anal. Chem. 2008, 80 , 657-664.

(24) Lee, J. H.; Song, Y.-A.; Han, J. Multiplexed proteomic sample preconcentration device using surface-patterned ion-selective membrane Lab on a Chip 2008, 8, 596-601.

(25) Kim, S. J.; Han, J. Self-Sealed Vertical Polymeric Nanoporous-Junctions for High-Throughput Nanofluidic Applications Anal. Chem. 2008, 80, 35073511.

(26) Wang, Y.-C.; Han, J. Pre-binding dynamic range and sensitivity enhancement for immuno-sensors using nanofluidic preconcentrator $L a b$ on a Chip 2008, 8, 392-394.

(27) Probstein, R. F. Physicochemical Hydrodynamics. An Introduction, 1989.

(28) Liu, Y.; Fanguy, J. C.; Bledsoe, J. M.; Henry, C. S. Dynamic coating using polyelectrolyte multilayers for chemical control of electroosmotic flow in capillary electrophoresis microchips Anal. Chem. 2000, 72, 5939-5944.

(29) Katayama, H.; Ishihama, Y.; Asakawa, N. Stable Cationic Capillary Coating with Successive Multiple lonic Polymer Layers for Capillary Electrophoresis Anal. Chem. 1998, 70, 5272-5277.

(30) Pinto, D. M.; Ning, Y.; Figeys, D. An enhanced microfluidic chip coupled to an electrospray Qstar mass spectrometer for protein identification Electrophoresis 2000, 21, 181-190. 
(31) Ro, K. W.; Chang, W.-J.; Kim, H.; Koo, Y.-M.; Hahn, J. H. Capillary electrochromatography and preconcentration of neutral compounds on poly(dimethylsiloxane) microchips Electrophoresis 2003, 24, 3253-3259.

(32) McClain, M. A.; Culbertson, C. T.; Jacobson, S. C.; Allbritton, N. L. S.; C. E.; Ramsey, J. M. Microfluidic Devices for the High-Throughput Chemical Analysis of Cells Anal Chem 2003, 75, 5646-5655.

(33) Razunguzwa, T. T.; Warrier, M.; Timperman, A. T. ESI-MS Compatible Permanent Coating of Glass Surfaces Using Poly(ethylene glycol)Terminated Alkoxysilanes for Capillary Zone Electrophoretic Protein Separations Anal. Chem. 2006, 78, 4326-4333.

(34) Moini, M.; Huang, H. Application of capillary electrophoresis/electrospray ionization-mass spectrometry to subcellular proteomics of Escherichia coli ribosomal proteins Electrophoresis 2004, 25, 1981-1987.

(35) David C. Simpson; Smith, R. D. Combining Capillary Electrophoresis with Mass Spectrometry for Applications in Proteomics Electrophoresis 2005, 26, 1291-1305.

(36) Eric Schiffer; Harald Mischak; Novak, J. High resolution proteome/peptidome analysis of body fluids by capillary electrophoresis coupled with MS Proteomics 2006, 6, 5615-5627.

(37) Ostuni, E.; Chapman, R. G.; Holmlin, R. E.; Takayama, S.; Whitesides, G. M. A Survey of Structure-Property Relationships of Surfaces that Resist the Adsorption of Protein Langmuir 2001, 17, 5605-5620.

(39) $\mathrm{Bi}, \mathrm{H}$. ; Meng, S.; Li, Y.; Guo, K.; Chen, Y.; Kong, J.; Yang, P.; Zhong, W.; Liu, B. Deposition of PEG onto PMMA microchannel surface to minimize nonspecific adsorption Lab on a Chip 2006, 6, 769-775.

(40) Uchida, K.; Otsuka, H.; Kaneko, M.; Kataoka, K.; Nagasaki, Y. A Reactive Poly(ethylene glycol) Layer To Achieve Specific Surface Plasmon Resonance Sensing with a High S/N Ratio: The Substantial Role of a Short Underbrushed PEG Layer in MInimizing Nonspecific Adsorption Anal Chem 2005, 77, 1075-1080.

(41) Liu, J.; Pan, T.; Woolley, A. T.; Lee, M. L. Suface-modified poly(methyl methacrylate) capillary electrophoresis microchips for protein and peptide analysis Anal Chem 2004, 76, 6948-6955.

(42) Razunguzwa, T. T.; Timperman, A. T. Fabrication and Characterization of a Fritless Microfabricated Electroosmotic Pump with Reduced pH Dependence Anal Chem 2004, 76, 1336-1341.

(43) Bard, A. J.; Faulkner, L. R. Electrochemical Methods, Fundamentals and Applications, 2nd ed.; John Wiley \& Sons, 2001.

(44) Kim, S. J.; Wang, Y.-C.; Lee, J. H.; Jang, H.; Han, J. Concentration polarization and nonlinear electrokinetic flow near a nanofluidic channel Physical Review Letters 2007, 99, 044501/044501-044501/044504.

(45) Wu, D.; Steckl, A. J. High speed nanofluidic protein accumulator Lab on a Chip 2009, 9, 1890-1896. 
46) Kim, P.; Kim, S. J.; Han, J.; Suh, K. Y. Stabilization of lon Concentration Polarization Using a Heterogeneous Nanoporous Junction Nano Lett 2010, 10 16-23. 


\title{
Chapter 5
}

\section{Positively Coated Nuclear Track Etched Nanocapillary Membranes for Enrichment of Cations}

\begin{abstract}
In this work, nanocapillary membranes are coated with a multilayer polyelectrolyte coating to create nanocapillary membranes with positive surface charges. The coatings are characterized by observing the movement of three probe molecules of different charges (positive, neutral, and negative) through the nanocapillary membranes under current limited conditions. The transport of the probe molecules indicates that the coated nanocapillary membranes are successfully altered from a net negative surface charge to a net positive surface charge. The transport of the probe molecules also indicates that the nanocapillary membrane became anion permselective. The magnitudes of the electroosmotic flow, determined from the transport of the neutral probe molecule, are compared between uncoated and coated nanocapillary membranes and the coated nanocapillary membranes produce anodic EOF with one-third less electroosmotic flow than the uncoated nanocapillary membranes.
\end{abstract}




\subsection{Introduction}

Multiplexed systems with integrated sample preparation, manipulation, and detection methods offer much hope to fields including proteomics, metabolomics, and clinical analysis. One important aspect of these multiplexed systems are methods to enrich samples to ensure their detection at such small volumes. Several analyte concentrators have been developed for integrated microfluidic devices and of particular relevance to this work are devices that rely on ion permselective materials that generate concentration polarization. ${ }^{1-20}$ Concentration polarization ${ }^{21}(\mathrm{CP})$ arises from the presence of an ion permselective material, media that preferentially transports either cations or anions, with an applied force and in the context of this work an electric field. One method of developing CP has been observed to occur when a nanocapillary membrane (NCM) or nanochannel has double layer overlap that causes it to behave as an ion permselective material. ${ }^{22}$ For example, if the media preferentially transports anions, the anions accumulate on the anode side of the media and become depleted on the cathode side. Since cations are restricted from passing through the NCM, the cations cannot remain near the face of the NCM either on the cathode side to uphold electroneutrality within the system. Rather than passing through the NCM, cations move away from the NCM. Therefore, both cations and anions become depleted on the same side of the media. The anode side of the media must maintain electroneutrality as well, so cations migrate into the region of the accumulating anions. To summarize the overall result, one side of the media becomes enriched while the other side becomes depleted of ions.

The occurrence of $\mathrm{CP}$ has been used to create analyte concentrators in microfluidic devices. In several devices, a permselective nanofluidic element is incorporated into the microfluidic device and as an electric field is applied CP becomes established and at the edge of the $\mathrm{CP}$ depleted zone a front forms where sample becomes enriched. ${ }^{2,3,5-7,9,10,12,14-16,18,19,23}$ Thus far, the majority of these analyte concentrators have focused on anionic samples and incorporated cation permselective materials primarily because most materials 
have a negative surface charge natively and are by default cation permselective. Although many references did not investigate cationic samples, several reports show that devices utilizing $\mathrm{CP}$ to enrich analyte are unable to enrich positive samples with cation permselective materials. 5, 10, 13, 24 However, a few authors have been able to enrich a positive sample while implementing a cation permselective NCM that induced CP. 4, 9, 23 Additionally, Hoeman et al. developed a device that created an enriched plug of their anionic sample, but also exhibited an increase in cations within the nanofluidic element. ${ }^{15}$ While not all the devices enrich analyte under the same polarities and have various differences, little discussion in the literature offers an explanation as to why some devices enrich positive samples and others cannot.

It is advantageous to develop a reliable concentrator capable of enriching positive samples rather than only negative samples. For application to proteomic samples, a device must be capable of accommodating both positive and negative samples due to the diversity among proteins and peptides that arise from the twenty different amino acids in various combinations that make up protein and peptide sequences. It appears there is much ambiguity within the literature on the device characteristics necessary to develop an analyte concentrator for cationic samples. This work aims to develop an anion permselective material to be implemented as the nanofluidic element within a cationic concentrator, which has yet to be accomplished in the literature.

The anion permselective material is developed by coating an existing nanofluidic media to change the surface charge from negative to positive. In order to avoid coating the entire device, an NCM can be implemented as the nanofluidic element and altered separately without affecting the surface charge of the microfluidic device. ${ }^{1-3,16}$ Multiple coating methods have been developed and two major categories exist: those that are dynamic coatings and those that are permanent coatings. Dynamic coatings interact with the surface through nonspecific adsorption and electrostatic interactions while permanent coatings are either created through physical adsorption or covalent linkages between the substrate surface and the coating molecules. Dynamic coatings are typically 
easy to form and do not require precise control of environmental conditions while permanent coatings are typically more sensitive to environmental conditions and are more difficult to create. Dynamic coatings require that the modifier used to coat the surface be dissolved in the running buffer, which is often incompatible with mass spectrometry. Dynamic coatings establish an equilibrium with the solution and therefore the modifier must be present in the running buffer to balance the equilibrium. Permanent coatings do not have a dynamic equilibrium and therefore do not require the surface modifier to be dissolved in the running buffer. Permanent coatings are usually longer lasting, more robust, and compatible with mass spectrometry.

Once a coating has been formed, the coating must be characterized in order to ensure the NCM has been effectively coated and the surface charge altered to a net positive surface charge. Several publications have characterized the transport through nanosized channels. ${ }^{25-30}$ Previously, the transport through NCMs has been characterized by monitoring the movement of probe molecules of different charge through the NCM with varying electric fields. ${ }^{25,26}$ The permeabilities of three different probe molecules with different ionic strengths were compared as well. A similar approach was used to characterize carbon nanotubes and included a method to calculate the electroosmotic flow from the data, which can be useful to quantitatively characterize a coating. ${ }^{27}$

A commonly used permanent coating, formed through physical adsorption, to alter the surface charge is polybrene, which has found applications in capillary electrophoresis (CE) ${ }^{31-38}$ However, the coating has a tendency to degrade over time leading to unstable EOF. To improve the stability of the polybrene dynamic coating, integration of multiple layers on the surface has been performed. ${ }^{37}$ The multilayer coatings are created by alternating between positive and negative polymers and the final layer should have the desired surface charge. Chen et al. fabricated a polycarbonate microfluidic device and successfully coated it with a single polybrene layer to form a positive surface charge and reverse the electroosmotic flow (EOF) of the CE separation. ${ }^{38}$ However, the work reported within this document is the first report of implementing the polybrene coating 
technique on a polycarbonate NCM and the first time the multilayer coating method is used with a polycarbonate substrate.

In this work, the net surface charge of a polycarbonate NCM is altered from negative to positive by implementing a multilayer polyelectrolyte coating. The movement of positive, neutral, and negative probe molecules are monitored as a function of the applied voltage under current limited conditions and qualitatively compared to uncoated NCMs. The electroosmotic flow is calculated for each NCM based on the transport of the neutral probe molecule, riboflavin.

\subsection{Experimental}

\subsubsection{Chemicals}

The following chemicals were purchased from Sigma Aldrich (Milwaukee, WI): sodium phosphate, fluorescein sodium salt, rhodamine 123 , riboflavin, dextran sulfate sodium salt from Leuonostoc spp (average molecular weight is greater than 500,000), hexadimethrine bromide. Methanol, sodium hydroxide $(\mathrm{NaOH})$, sodium chloride $(\mathrm{NaCl})$, and sodium dodecyl sulfate (SDS) were purchased from Fisher Scientific (Fair Lawn, NJ).

\subsubsection{NCM Preparation and Coating}

The polycarbonate nuclear track-etched NCMs with nominal pore sizes of $10 \mathrm{~nm}$ (GE Osmonics, Minnetonka, MN) were cut to $3 / 8$ " in diameter using a shim stock punch (McMaster Carr, Aurora, $\mathrm{OH}$ ). Several NCMs cut to size were placed in a $1 \mathrm{M} \mathrm{NaOH}$ solution containing $0.1 \%(\mathrm{v} / \mathrm{v})$ SDS for 30 minutes. Subsequently, the NCMs were transferred to a solution of deionized water (nominally $18.3 \mathrm{M} \Omega \cdot \mathrm{cm}$ ), prepared with a Barnstead Nanopure Infinity water purifier (Dubuque, IA) for 30 minutes. The NCMs were then placed in a solution of $50 \mathrm{mM} \mathrm{NaCl}$ containing $5 \%$ hexadimethrine bromide for 30 minutes. Next, the NCMs were removed and allowed to set out to dry for 30 minutes in the atmosphere under ambient conditions. The NCMs were rinsed with deionized water and placed in a $50 \mathrm{mM} \mathrm{NaCl}$ solution containing 3\% dextran sulfate and left for 30 minutes. Following the coating with the dextran sulfate, the NCMs set 
to dry for 30 minutes and were rinsed with deionized water. The coating with the hexadimethrine bromide was repeated in the same manner as described to result in a final positive layer.

To prepare the NCMs to be characterized, an area had to be defined reproducibly to characterize the transport through the NCM. An NCM with 3/8" diameter was mounted and sealed between two pieces of tape with 3/16" holes allowing for the same amount of NCM to be exposed to solution for each experiment.

\subsubsection{Instrumental setup}

The permeation cell was made in house with four compartments as shown in Figure 5.1.

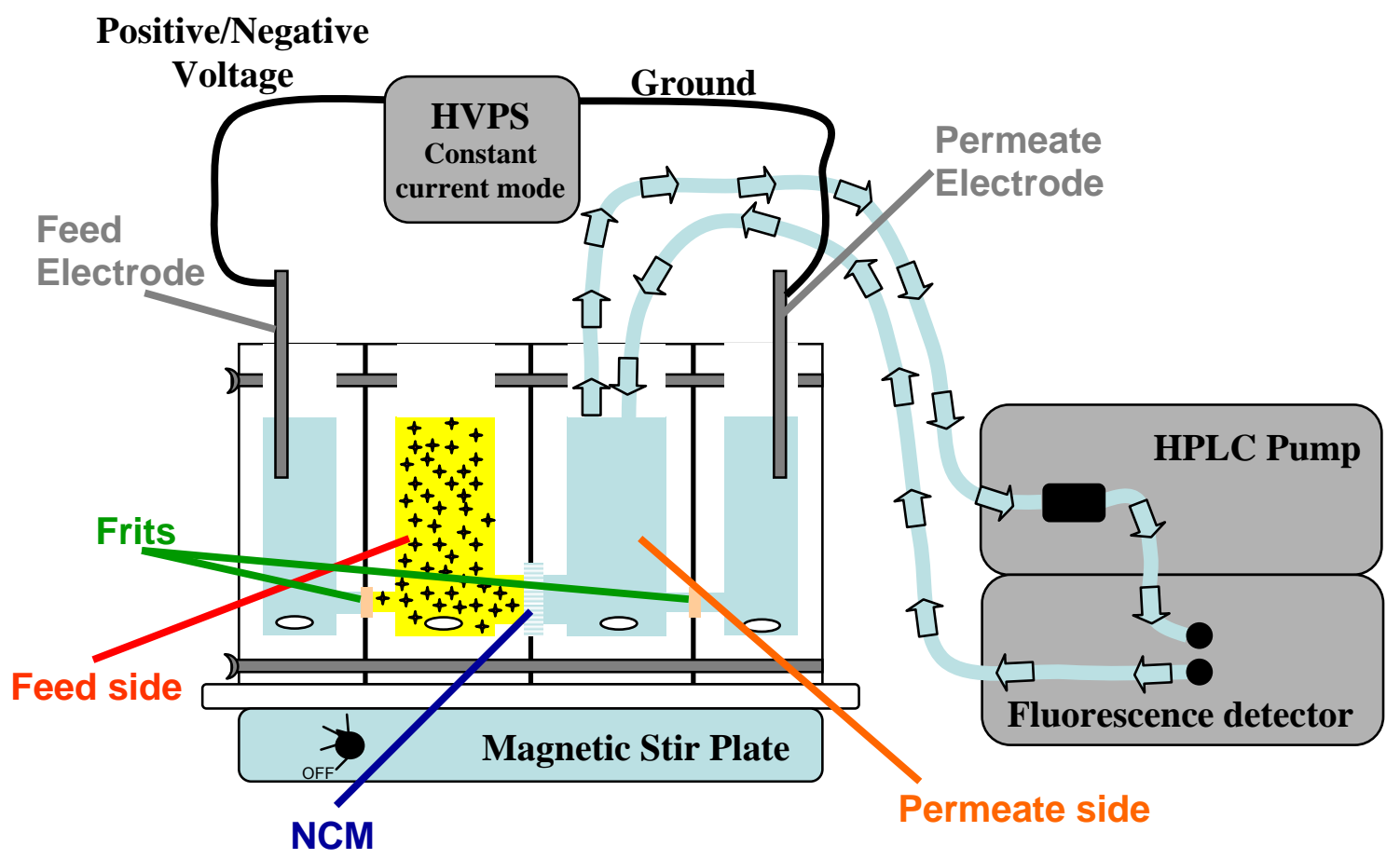

Figure 5.1: Schematic representation of the experimental setup. The feed side of the NCM has a high concentration of probe molecule while the permeate side has a low concentration (initially zero) of probe molecule. The transport of the probe molecule through the NCM is monitored by pumping the permeate solution through a fluorescence detector and monitoring the fluorescence intensity over time. 
A compartment with an electrode was at one end with the adjacent compartment separated by a frit containing buffer solution with analyte. The NCM was placed between the second and third compartments. The third compartment contained buffer solution separated with a frit from a fourth compartment with the second electrode at the end. The two compartments that housed platinum electrodes were separated from their adjoining compartments with frits to minimize affects from electrolysis. The NCM mounted between tape was placed between a compartment containing $10 \mathrm{mM}$ sodium phosphate buffer with a $\mathrm{pH} 7$ and either $10 \mu \mathrm{M}$ fluorescein, $50 \mu \mathrm{M}$ riboflavin, or $10 \mu \mathrm{M}$ rhodamine 123 and a compartment containing only $10 \mathrm{mM}$ phosphate buffer. Experiments that involved rhodamine 123 had $5 \%$ methanol added to the buffer to ensure the solubility of the dye. A stir bar was placed in each compartment to ensure uniform solution concentrations throughout the experiments. The permeation cell was placed on a stir plate and the fluorescence of the compartment containing only buffer and no electrodes was monitored by pumping solution through an Agilent 1100 series fluorescence detector at $5 \mathrm{~mL} / \mathrm{min}$ with an Agilent 1100 (Santa Clara, CA) series binary pump and back into the same compartment.

A 610E Trek HV power supply (Medina, NY) was connected to the electrodes of the permeation cell and operated in constant current mode at 0 , $200,+200,+400,-400$, and $0 \mu \mathrm{A}$ using this sequence throughout the experiments. The electrode closest to the permeate side of the permeation cell is always held at ground while the polarity and magnitude of the voltage was changed on the feed side of the permeation cell. The current and voltage were monitored throughout the experiments by using the voltage monitor output and current monitor outputs from the high voltage power supply. Coaxial cables were connected to both of these outputs and $1 \mathrm{~V}$ at the voltage monitor output represented $1000 \mathrm{~V}$ of high voltage output and $1 \mathrm{~V}$ at the current monitor output represented $200 \mu \mathrm{A}$ of load current. The currents and voltages were recorded on a computer using a personal measurement device model 1608FS (Measurement Computing Norton, MA) and the software provided with the personal measurement device was used for recording the data. The personal 
measurement device was used to convert the current and voltage measurements to *.csv files which were then imported into Microsoft Excel for data analysis. The fluorescence with respect to time was monitored using the analog output from the fluorescence detector and using a PC-interfaced digital multimeter (RadioShack, Fort Worth, TX) and Meterview version 1.0 to record the data. For analyses implementing fluorescein and rhodamine 123 , the excitation was set at $490 \mathrm{~nm}$ and the emission was at $520 \mathrm{~nm}$ with the photomultiplier tube gain set to 9. For analyses using riboflavin, the excitation was set to $450 \mathrm{~nm}$ and the emission was set to $525 \mathrm{~nm}$ with a gain of 11 .

\subsubsection{Calibration Curve Determination}

Calibration curves for each probe molecule were obtained by first placing $10 \mathrm{~mL}$ of blank or buffer solution in a scintillation vial. The intensity of the blank solution and subsequent standards were measured using the same settings as used for the experiments. The measurement was acquired as the buffer was pumped through the detector at $5 \mathrm{~mL} / \mathrm{min}$ and back into the scintillation vial. To obtain each data point for the calibration curve, standard was spiked directly into the scintillation vial and the intensity was allowed to reach a steady value. The average intensity was determined for each standard and background subtracted and correlated to the concentration of the standard after accounting for the dilution.

\subsection{Results and Discussion}

An easy method to characterize the success of the coating process is to determine whether the EOF is reversed. NCMs with positive surface charge are expected to pump toward the anode because the double layer has a net negative charge. In the case of the uncoated NCMs with a negative surface charge, the EOF is expected to be toward the cathode because the double layer has a net positive charge. To determine the amount of probe molecule transported through the NCMs, a calibration curve for each probe molecule as shown in Figure 5.2 is constructed before the NCMs are tested. 


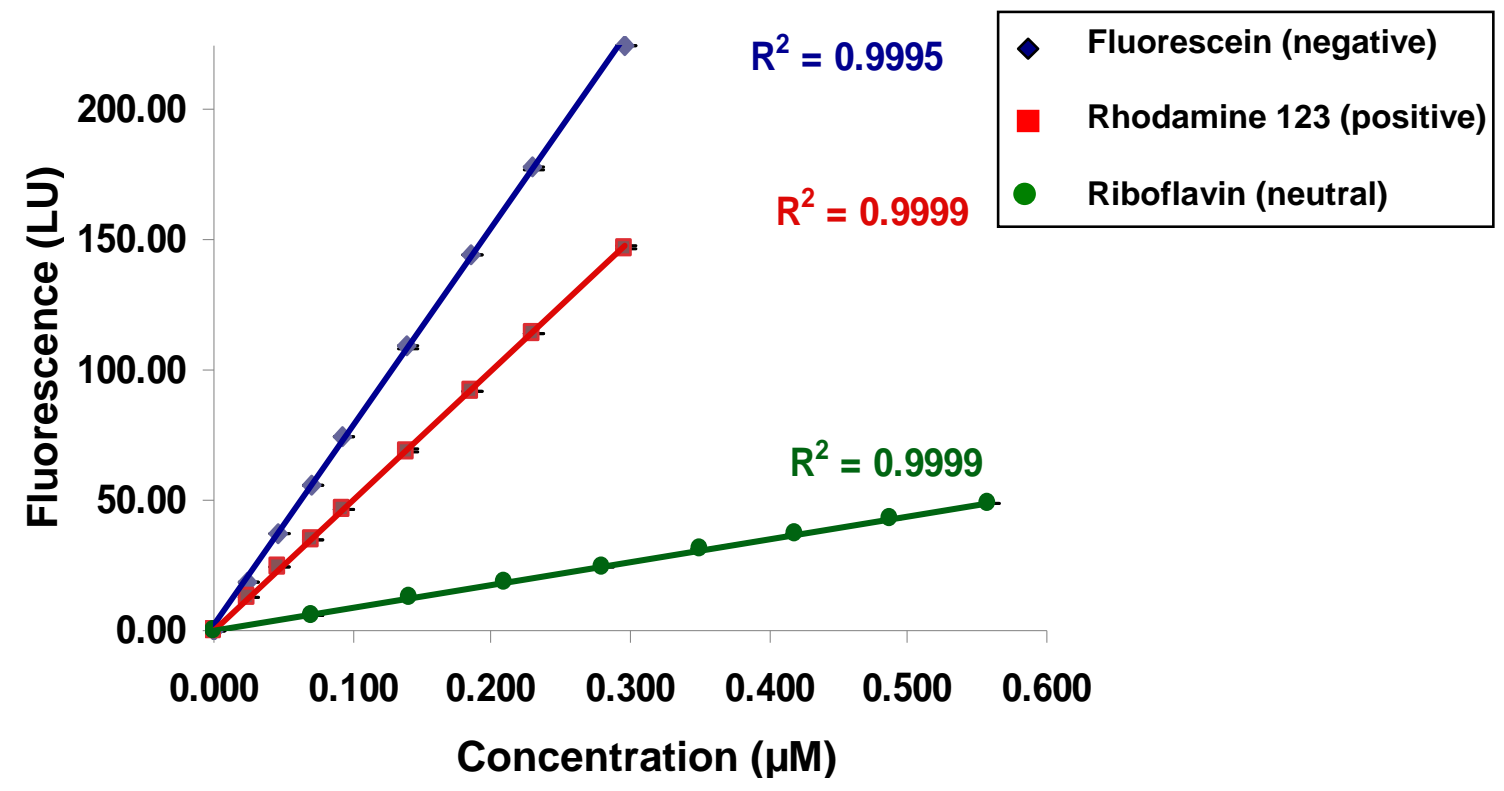

Figure 5.2: Calibration curve for the fluorescence produced from negative fluorescein (blue diamonds), positive rhodamine 123 (red squares), and neutral riboflavin (green triangles) at given concentrations.

The applied voltage under current limited conditions is varied as the transport through the NCM is monitored and graphed as six regions that correspond to different applied currents as shown in Figure 5.3. Initially, no current is applied and therefore the only mechanism for the probe molecule to pass through the NCM is diffusion. The diffusion of riboflavin through both the uncoated and coated NCM is low as expected. In the next region, $-200 \mu \mathrm{A}$ is applied and the passage of riboflavin through the coated NCM, toward the anode, increases significantly while the transport of riboflavin through the uncoated NCM decreases under the same conditions. Note that in Figure 5.3 different $y$-axis scales are used to present the coated and uncoated data to ensure the details of the data can be visualized, but the slopes of each region are provided to illustrate that the changes in slope are evident and not a result of differences in the $y$-axes used. (Figures $5.4 \& 5.5$ present data in the same format) The regions corresponding to transport toward the permeate side always have a greater slope than the regions corresponding to transport toward the feed side. The transport toward the permeate side causes the concentration in the 
permeate side to increase and gives a greater change than the transport toward the feed side that is measuring a decrease in concentration. The riboflavin migrates toward the anode in the case of the coated NCM and toward the cathode in the uncoated NCM. The only mechanisms present to transport the riboflavin are diffusion and EOF since the riboflavin is a neutral molecule. The diffusion is in the same direction (toward the permeate side) for both uncoated and coated NCMs and thus the EOF must be in opposite directions to result in the transport of riboflavin to the permeate side of the cell under opposite polarity voltages. Therefore, the overall surface charge of the NCM has been changed to positive as indicated by the anodic EOF.

Further conformation of the coating is observed with the application of the remaining current values. When $+200 \mu \mathrm{A}$ is applied, the transport of the riboflavin through the coated NCM quickly decreases below the diffusion rate as the slope of the diffusion portion of the curve is greater than the slope where a positive current is applied as seen in the table within Figure 5.3. A sharp increase in the transport of riboflavin through the uncoated NCM is observed in the $+200 \mu$ A region of the curve. As expected, the transport increases even more for the uncoated NCM when the current is increased to $+400 \mu \mathrm{A}$ and the transport through the coated NCM is still extremely low. When the voltage in current limited mode is switched back to the negative polarity, the coated NCM immediately has an increased transport of riboflavin and the uncoated NCM sharply decreases in transport of riboflavin. 


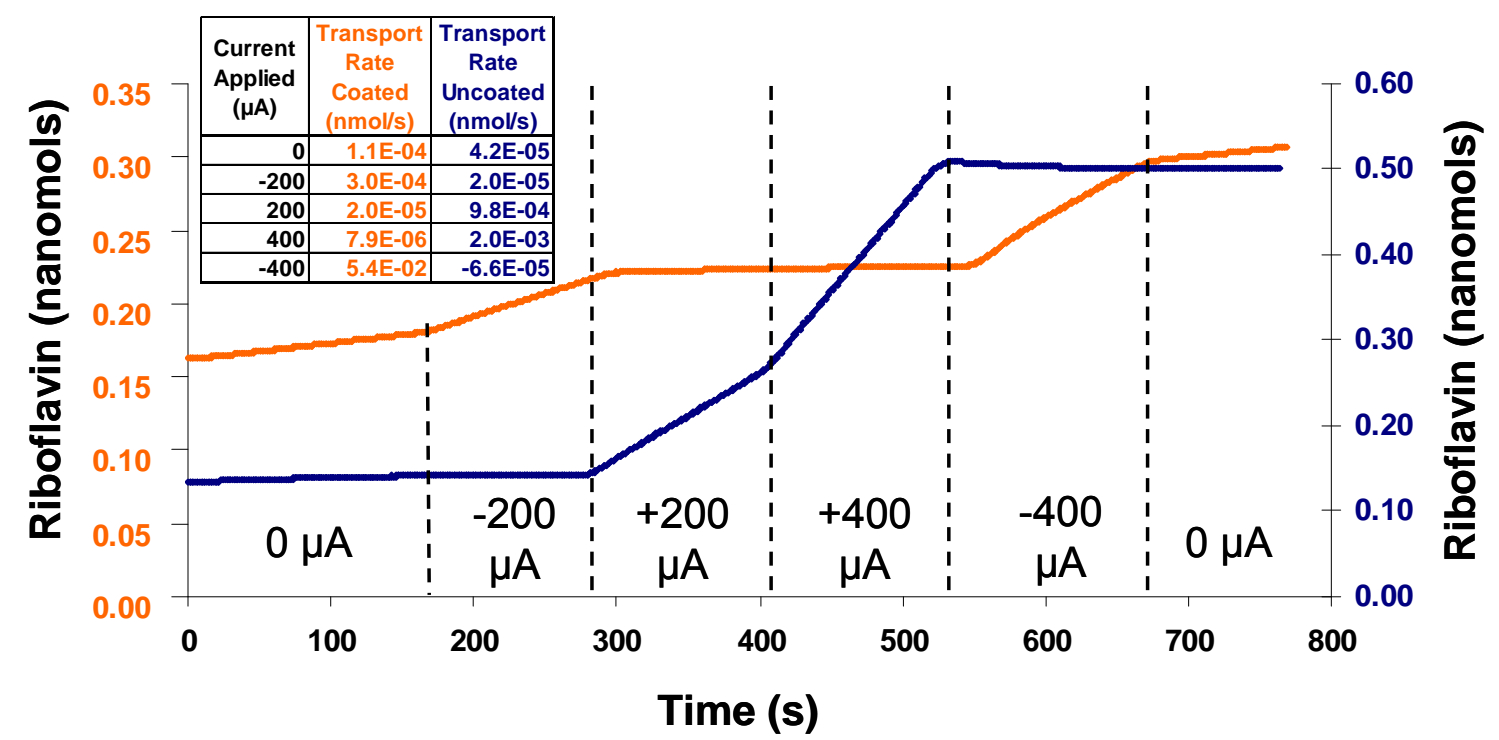

Coated (positive surface)

- Uncoated (negative surface)

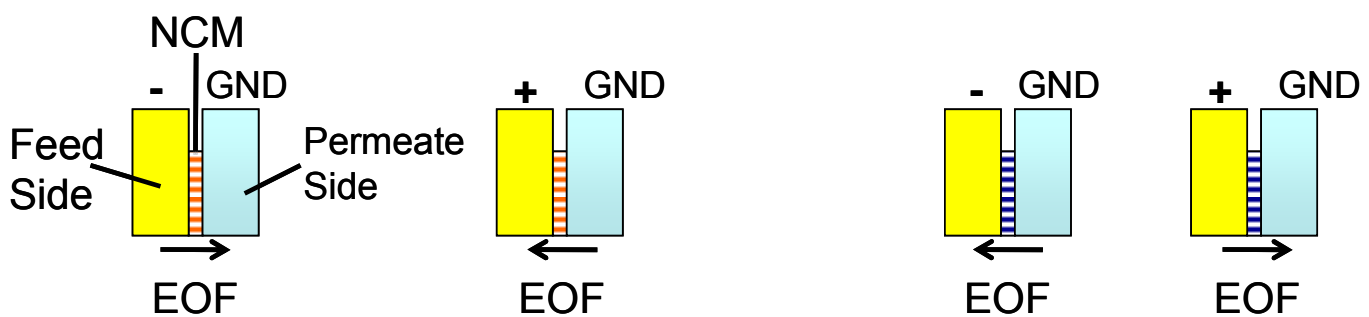

Figure 5.3: Representative transport of a neutral marker, riboflavin, through uncoated (blue) and coated (orange) NCMs in response to applied currents. The NCMs have nominal pore sizes of $10 \mathrm{~nm}$ and $10 \mathrm{mM}$ phosphate buffer at $\mathrm{pH} 7$ is used. The y-axis for the coated NCM appears on the left and the $y$-axis for the uncoated NCM appears on the right. In the inset, a table displays the slopes of the curves within each applied current region for both the coated and uncoated NCMs. A simplified schematic of the permeation cell is provided below the graph to aid in visualizing the flow through the coated and uncoated NCMs as the potential is switched.

Characterization of the transport of a model anion, fluorescein, through negatively and positively coated NCMs is compared in Figure 5.4. Fluorescein is a negatively charged compound and therefore it will tend to electrophoretically migrate to the anode in addition to the diffusive and EOF transport. Depending on the direction and strength of the EOF, the electrophoretic migration of fluorescein may be enhanced or hindered.

The positive coated NCM transports a much greater amount of fluorescein than the uncoated NCM as shown in Figure 5.4. The uncoated NCM allows a 
much smaller amount of fluorescein to be transported through the NCM and a separate $y$-axis had to be used in order to see the details of the data (note the scale is a factor of ten less). The EOF of the uncoated NCM opposes the electrophoretic transport of fluorescein while the EOF of the coated NCM enhances the electrophoretic transport of fluorescein. Another interesting point to note is both the coated and uncoated NCMs show an increase in transport of fluorescein under the negative applied currents and decrease in transport under the positive applied currents. This trend results because the electrophoretic migration of fluorescein is the dominate mechanism of transport for fluorescein through the NCM, despite the EOF of the uncoated NCMs opposing the electrophoretic transport of fluorescein. Finally, the increased transport of fluorescein through the coated NCM indicates that the desired anion permselectivity has been created. 


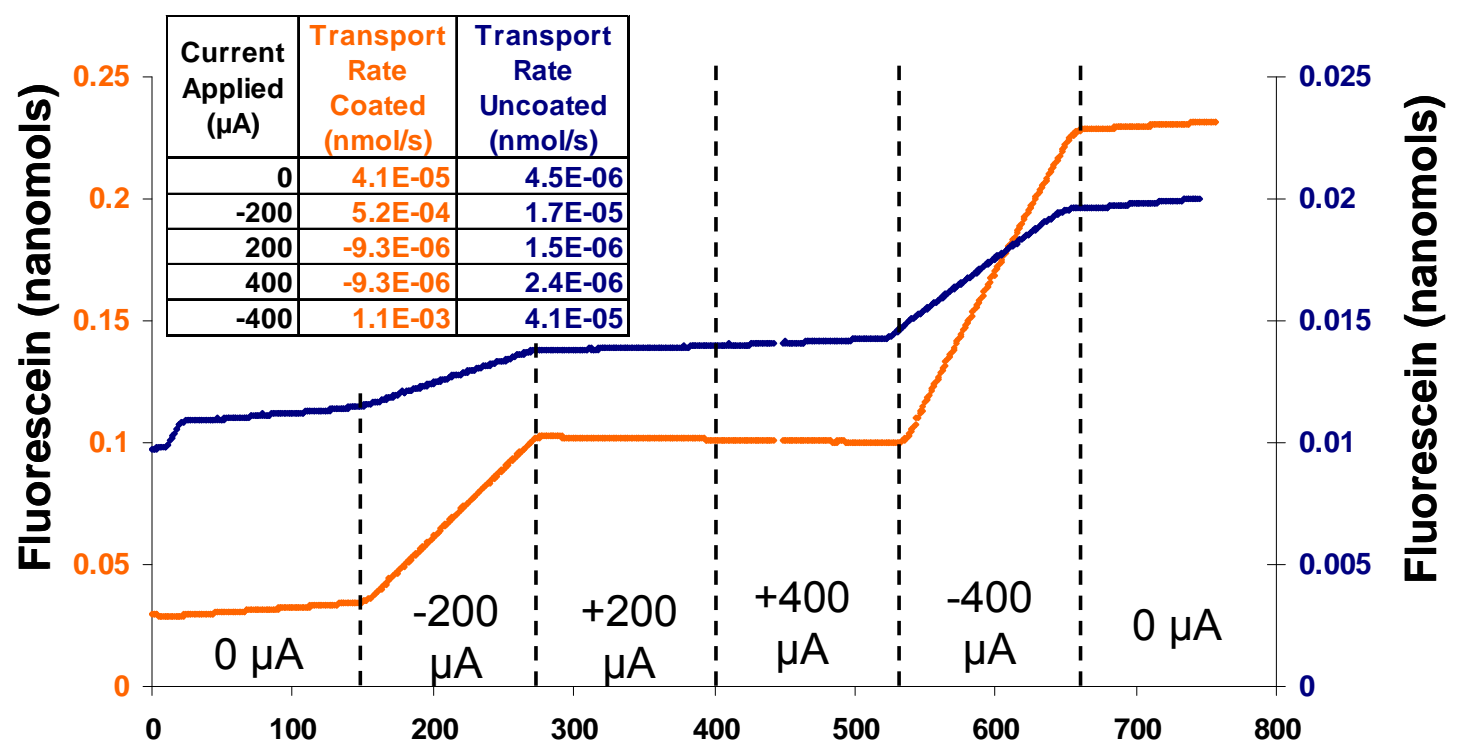

Coated (positive surface)

Time (s)

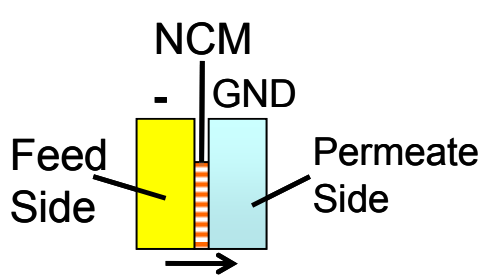

EOF

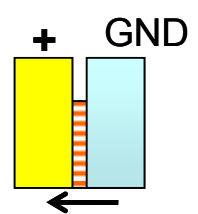

EOF

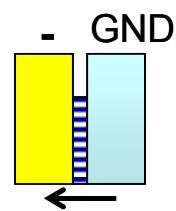

EOF

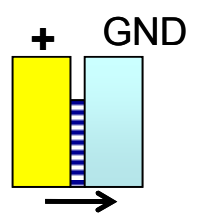

EOF

Figure 5.4: Representative transport of a negative marker, fluorescein, through uncoated (blue) and coated (orange) NCMs in response to applied currents. The NCMs have nominal pore sizes of $10 \mathrm{~nm}$ and $10 \mathrm{mM}$ phosphate buffer at $\mathrm{pH} 7$ is used. The y-axis for the coated NCM appears on the left and the y-axis for the uncoated NCM appears on the right. In the inset, a table displays the slopes of the curves within each applied current region for both the coated and uncoated NCMs. Again, a simplified schematic of the permeation cell is provided below the graph to aid in visualizing the flow through the coated and uncoated NCMs as the potential is switched.

Figure 5.5 compares the transport of the positive organic dye, rhodamine 123 , through the uncoated and coated NCMs. Similar to fluorescein, rhodamine 123 also has three possible transport mechanisms involved: diffusion, EOF, and electrophoresis. However, rhodamine 123 is positive and will tend to migrate toward the cathode rather than the anode. Both the uncoated and coated NCMs show a decrease in transport of rhodamine 123 at negative applied currents and 
an increase in transport of rhodamine 123 at positive applied currents. In this case, the EOF of the coated NCM opposes the electrophoretic transport of rhodamine 123 while the electrophoretic migration of rhodamine 123 dominates as the mechanism to transport rhodamine 123 through the coated NCM. As seen in Figure 5.5, the uncoated NCM has increased transport of rhodamine 123 compared to the coated NCM at positive applied currents. Again separate y-axis scales were used for the coated and uncoated NCMs and in this case the coated NCM has a y-axis that is a factor of two less than the y-axis for the uncoated NCM. Therefore, the coated NCM has a suppressed transport of rhodamine 123 compared to the uncoated NCM. 


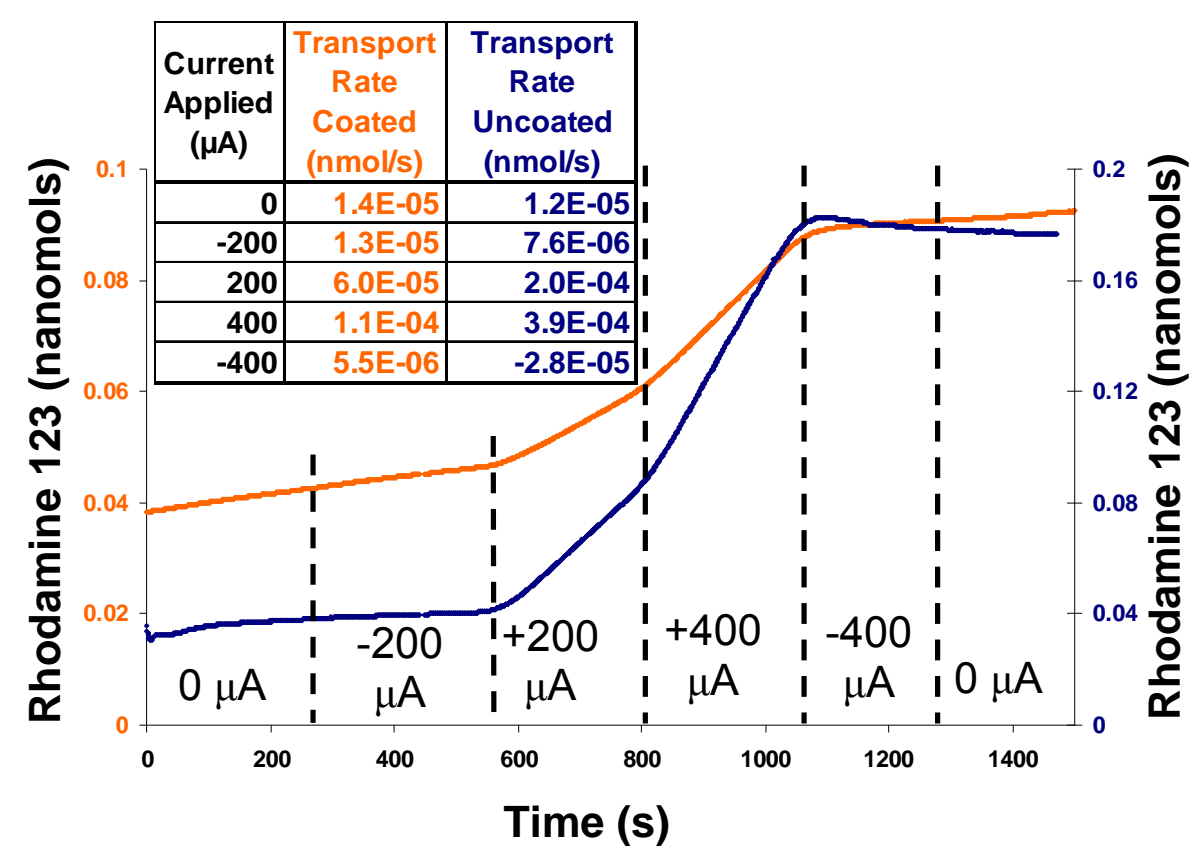

Coated (positive surface)

Uncoated (negative surface)
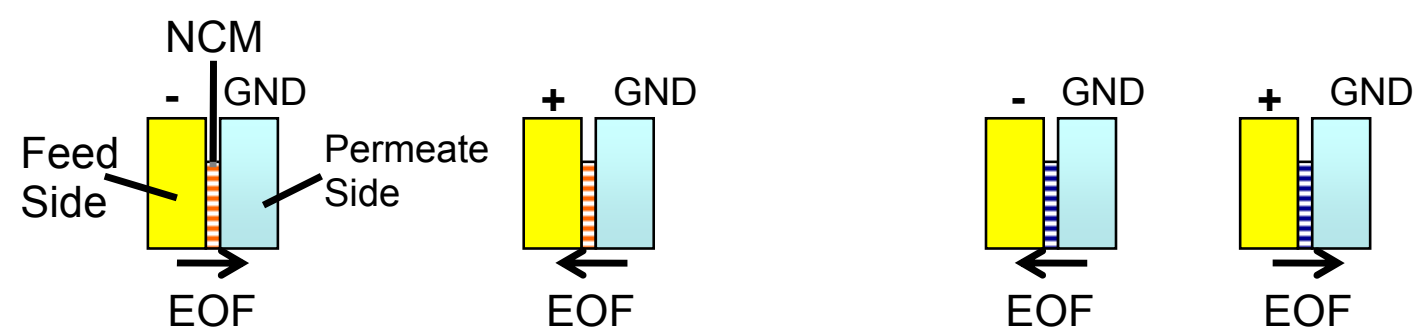

Figure 5.5: Representative transport of a positive marker, rhodamine 123, through uncoated (blue) and coated (orange) NCMs in response to applied currents. The NCMs have nominal pore sizes of $10 \mathrm{~nm}$ and $10 \mathrm{mM}$ phosphate buffer at $\mathrm{pH} 7$ is used with $5 \%$ methanol. The y-axis for the coated NCM appears on the left and the y-axis for the uncoated NCM appears on the right. In the inset, a table displays the slopes of the curves within each applied current region for both the coated and uncoated NCMs. Again, a simplified schematic of the permeation cell is provided below the graph to aid in visualizing the flow through the coated and uncoated NCMs as the potential is switched.

The permselectivity of the coated NCMs are determined by comparing the transport of the negative and positive probe molecules through the coated NCMs. It should be noted that the electrophoretic mobilities of the two probe molecules have been derived from a study involving a coated capillary and are such that the positive probe molecule is transported only 1.08 times faster than the negative probe molecule. ${ }^{39}$ In Figures $5.4 \& 5.5$, tables are provided that show the 
transport rate of each probe molecule. Since both the transport of the negative and positive are dominated by their electrophoretic mobilities rather than the EOF, the transport rates will be compared under opposite polarities. At a magnitude of $400 \mu \mathrm{A}$, the negative is transported ten times faster than the positive through the coated NCM. This data provides additional proof that the NCM has been successfully changed from a cation permselective NCM to an anion permselective NCM. The transport ratio of 10 shows anion permselectivity, but the degree of anion permselectivity is very small. The rate of transport of the positive through the uncoated NCM at $400 \mu \mathrm{A}$ is 163 times faster than the negative through the uncoated NCM. Therefore, the uncoated NCM is a more permselective media than the coated NCM, indicating that the native surface has a higher charge density and zeta potential than the coated surface.

Thus far, only qualitative comparisons have been discussed between the coated and uncoated NCMs. From the data collected about the transport of riboflavin, the EOF produced by each NCM can be calculated. The calculated EOF values presented in Table 5.1 have two obvious implications: 1) the reproducibility of the EOF from the coated NCMs is poor in comparison to the uncoated NCMs according to the values of the relative standard deviations and 2) the magnitude for the EOF produced by the coated NCM is approximately one-third the magnitude of EOF produced by the uncoated NCM.

Table 5.1: Comparison of Electroosmotic Flow produced by Coated and Uncoated NCMs.

\begin{tabular}{|c|c|c|c|c|}
\hline $\mathbf{N C M}$ & $\begin{array}{c}-\mathbf{2 0 0} \boldsymbol{\mu A} \text { Applied } \\
\text { Positive Coated } \\
\boldsymbol{v}_{\text {eo }}(\mathbf{m L} / \mathbf{C})\end{array}$ & $\begin{array}{c}+\mathbf{2 0 0} \boldsymbol{\mu A} \text { Applied } \\
\text { Uncoated } \\
\boldsymbol{v}_{\text {eo }}(\mathbf{m L} / \mathrm{C})\end{array}$ & $\begin{array}{c}-\mathbf{4 0 0} \boldsymbol{\mu A} \text { Applied } \\
\text { Positive Coated } \\
\boldsymbol{v}_{\text {eo }}(\mathbf{m L} / \mathbf{C})\end{array}$ & $\begin{array}{c}+\mathbf{4 0 0} \boldsymbol{\mu A} \text { Applied } \\
\text { Uncoated } \\
\boldsymbol{v}_{\text {eo }}(\mathbf{m L} / \mathbf{C})\end{array}$ \\
\hline 1 & $2.58 \mathrm{E}-02$ & $1.04 \mathrm{E}-01$ & $2.34 \mathrm{E}-02$ & $1.09 \mathrm{E}-01$ \\
\hline 2 & $2.91 \mathrm{E}-02$ & $9.90 \mathrm{E}-02$ & $3.00 \mathrm{E}-02$ & $9.97 \mathrm{E}-02$ \\
\hline 3 & $1.62 \mathrm{E}-02$ & $1.04 \mathrm{E}-01$ & $1.49 \mathrm{E}-02$ & $9.57 \mathrm{E}-02$ \\
\hline 4 & $3.81 \mathrm{E}-02$ & $1.00 \mathrm{E}-01$ & $3.65 \mathrm{E}-02$ & $9.67 \mathrm{E}-02$ \\
\hline 5 & $3.87 \mathrm{E}-02$ & $9.75 \mathrm{E}-02$ & $3.54 \mathrm{E}-02$ & $9.58 \mathrm{E}-02$ \\
\hline Average & $2.96 \mathrm{E}-02$ & $1.01 \mathrm{E}-01$ & $2.80 \mathrm{E}-02$ & $9.94 \mathrm{E}-02$ \\
\hline Std. Dev. & $9.34 \mathrm{E}-03$ & $3.03 \mathrm{E}-03$ & $9.03 \mathrm{E}-03$ & $5.64 \mathrm{E}-03$ \\
\hline$\%$ RSD & 31.6 & 3.0 & 32.2 & 5.7 \\
\hline
\end{tabular}

The relative standard deviations are higher for the coated compared to the uncoated NCMs indicating that the coating process is a significant source of the 
error or uncertainty in the measurements. Note the EOF is reported in $\mathrm{mL} / \mathrm{C}$, because this allows for the EOF to be normalized to the amount of current passed through the membrane thereby eliminating any variation in membrane porosity as a factor.

The second observation about the measured EOF of the coated NCMs compared to the uncoated NCMs indicates the surface charge of the coated NCM is opposite but with a much reduced surface charge compared to the uncoated NCM. The greater the surface charge on the nanocapillaries the greater the ion permselectivity of the NCM. Therefore, coupling the NCM to a microfluidic device as performed previously may result in a less effective creation of concentration polarization (CP) that eventually leads to the formation of an enriched plug of analyte within the microchannel. The formation of $\mathrm{CP}$ is necessary to form an enriched plug of analyte within the device. However, it has been suggested previously that $\mathrm{CP}$ is a self-feeding process meaning that once it becomes established it drives the system into stronger $\mathrm{CP} .{ }^{40}$ Therefore, the decreased permselectivity may not be inhibitive to the performance of an NMI involving a multilayer polyelectrolyte coated NCM, as long as the membrane is permselective enough to induce $\mathrm{CP}$. Once $\mathrm{CP}$ occurs the system will continue to establish stronger CP through positive feedback leading to the formation of an enriched band of analyte.

\subsection{Conclusions}

The coating effectively changes the net surface charge of the NCM and therefore reverses the electroosmotic flow as shown by the transport of the neutral marker. However, the magnitude of the reversed EOF is one-third the magnitude of the EOF produced from the negative (uncoated) surface. Therefore, the ion permselectivity of the coated NCM is much lower than the uncoated NCM. The transport of the negative and positive probe molecules also indicates the reversal of the EOF and anion permselectivity of the NCM. The reproducibility of the EOF measurements for the coated NCMs is poor compared to the uncoated NCMs when five different NCMs were used to obtain 
electroosmotic flow values. The reproducibility of the coating method must be improved. 


\subsection{References}

(1) Zhang, Y.; Timperman, A. T. Integration of nanocapillary arrays into microfluidic devices for use as analyte concentrators, Analyst (Cambridge, United Kingdom) 2003, 128, 537-542.

(2) Miller, S. A.; Kelly, K. C.; Timperman, A. T. Ionic current rectification at a nanofluidic/microfluidic interface with an asymmetric microfluidic system, Lab on a Chip 2008, 8, 1729-1732.

(3) Kelly, K. C.; Miller, S. A.; Timperman, A. T. Investigation of Zone Migration in a Current Rectifying Nanofluidic/Microfluidic Analyte Concentrator, Anal. Chem. 2009, 81, 732-738.

(4) Pu, Q.; Yun, J.; Temkin, H.; Liu, S. Ion-Enrichment and Ion-Depletion Effect of Nanochannel Structures, Nano Lett 2004, 4, 1099-1103.

(5) Kim Sun, M.; Burns Mark, A.; Hasselbrink Ernest, F. Electrokinetic protein preconcentration using a simple glass/poly(dimethylsiloxane) microfluidic chip, Anal Chem 2006, 78, 4779-4785.

(6) Wang, Y.-C.; Stevens, A. L.; Han, J. Million-fold Preconcentration of Proteins and Peptides by Nanofluidic Filter, Anal Chem 2005, 77, 42934299.

(7) Lee, J. H.; Chung, S.; Kim, S. J.; Han, J. Poly(dimethylsiloxane)-Based Protein Preconcentration Using a Nanogap Generated by Junction Gap Breakdown, Anal Chem 2007, 79, 6868-6873.

(8) Dhopeshwarkar, R.; Crooks Richard, M.; Hlushkou, D.; Tallarek, U. Transient effects on microchannel electrokinetic filtering with an ionpermselective membrane, Anal Chem 2008, 80, 1039-1048.

(9) Huang, K.-D.; Yang, R.-J. A nanochannel-based concentrator utilizing the concentration polarization effect, Electrophoresis 2008, 29, 4862-4870.

(10) Yu, H.; Lu, Y.; Zhou, Y.-g.; Wang, F.-b.; He, F.-y.; Xia, X.-h. A simple, disposable microfluidic device for rapid protein concentration and purification via direct-printing, Lab on a Chip 2008, 8, 1496-1501.

(11) Wang, S.-C.; Wei, H.-H.; Chen, H.-P.; Tsai, M.-H.; Yu, C.-C.; Chang, H.-C. Dynamic superconcentration at critical-point double-layer gates of conducting nanoporous granules due to asymmetric tangential fluxes, Biomicrofluidics 2008, 2, 014102.

(12) Stein, D.; Deurvorst, Z.; Heyden, F. H. J. v. d.; Koopmans, W. J. A.; Gabel, A.; Dekker, C. Electrokinetic Concentration of DNA Polymers in Nanofluidic Channels, Nano Lett 2010, DOI: 10.1021/nl902228p.

(13) Yamamoto, S.; Hirakawa, S.; Suzuki, S. In Situ Fabrication of Ionic Polyacrylamide-Based Preconcentrator on a Simple Poly(methylmethacrylate) Microfluidic Chip for Capillary, Anal. Chem. 2008, 80, 8224-8230.

(14) Lee, J. H.; Song, Y.-A.; Tannenbaum, S. R.; Han, J. Increase of Reaction Rate and Sensitivity of Low-Abundance Enzyme Assay Using Micro/Nanofluidic Preconcentration Chip, Anal Chem 2008, 80, 31983204. 
(15) Hoeman, K. W.; Lange, J. J.; Roman, G. T.; Higgins, D. A.; Culbertson, C. T. Electrokinetic trapping using titania nanoporous membranes fabricated using sol-gel chemistry on microfluidic devices, Electrophoresis 2009, 30, 3160-3167.

(16) Zhou, K.; Kovarik, M. L.; Jacobson, S. C. Surface-Charge Induced Ion Depletion and Sample Stacking near Single Nanopores in Microfluidic Devices, J. Am. Chem. Soc. 2008, 130, 8614-8616.

(17) Kovarik, M. L.; Jacobson, S. C. Integrated Nanopore/Microchannel Devices for ac Electrokinetic Trapping of Particles, Anal. Chem. 2008, 80, 657-664.

(18) Lee, J. H.; Song, Y.-A.; Han, J. Multiplexed proteomic sample preconcentration device using surface-patterned ion-selective membrane, Lab on a Chip 2008, 8, 596-601.

(19) Kim, S. J.; Han, J. Self-Sealed Vertical Polymeric Nanoporous-Junctions for High-Throughput Nanofluidic Applications, Anal. Chem. 2008, 80, 3507-3511.

(20) Wang, Y.-C.; Han, J. Pre-binding dynamic range and sensitivity enhancement for immuno-sensors using nanofluidic preconcentrator, Lab on a Chip 2008, 8, 392-394.

(21) Probstein, R. F. Physicochemical Hydrodynamics. An Introduction, 1989.

(22) Plecis, A.; Schoch Reto, B.; Renaud, P. Ionic transport phenomena in nanofluidics: experimental and theoretical study of the exclusionenrichment effect on a chip, Nano Lett 2005, 5, 1147-1155.

(23) Huang, K.-D.; Yang, R.-J. Formation of ionic depletion/enrichment zones in a hybrid micro-/nano-channel, Microfluid Nanofluid 2008, 5, 631-638.

(24) Zhang, B.; Liu, H.; Karger, B. L.; Foret, F. Microfabricated Devices for Capillary Electrophoresis-Electrospray Mass Spectrometry, Anal Chem 1999, 71, 3258-3264.

(25) Kemery, P. J.; Steehler, J. K.; Bohn, P. W. Electric field mediated transport in nanometer diameter channels, Langmuir 1998, 14, 28842889.

(26) Kuo, T.-C.; Sloan, L. A.; Sweedler, J. V.; Bohn, P. W. Manipulating Molecular Transport through Nanoporous Membranes by Control of Electrokinetic Flow: Effect of Surface Charge Density and Debye Length, Langmuir 2001, 17, 6298-6303.

(27) Miller, S. A.; Young, V. Y.; Martin, C. R. Electroosmotic flow in templateprepared carbon nanotube membranes, Journal of the American Chemical Society 2001, 123, 12335-12342.

(28) Bath, B. D.; White, H. S. Electrically Facilitated Molecular Transport Analysis of the Relative Contributions of Diffusion, Migration, and Electroosmosis to Solute Transport in an Ion-Exchange Membrane, Anal. Chem. 2000, 72, 433-442.

(29) Bath, B. D.; Lee, R. D.; White, H. S. Imaging Molecular Transport in Porous Membranes. Observation and Analysis of Electroosmotic Flow in Individual Pores Using the Scanning Electrochemical Microscope, Anal. Chem. 1998, 70, 1047-1058. 
(30) Chen, W.; Yuan, J.-H.; Xia, X.-H. Characterization and Manipulation of the Electroosmotic Flow in Porous Anodic Alumina Membranes, Anal. Chem. 2005, 77, 8102-8108.

(31) Liu, X.; Erickson, D.; Li, D.; Krull, U. J. Cationic polymer coatings for design of electroosmotic flow and control of DNA adsorption, Anal. Chim. Acta 2004, 507, 55-62.

(32) Katayama, H.; Ishihama, Y.; Asakawa, N. Development of novel capillary coating based on physical adsorption for capillary electrophoresis, Anal. Sci. 1998, 14, 407-408.

(33) Liu, Y.; Fanguy, J. C.; Bledsoe, J. M.; Henry, C. S. Dynamic coating using polyelectrolyte multilayers for chemical control of electroosmotic flow in capillary electrophoresis microchips, Anal. Chem. 2000, 72, 5939-5944.

(34) Le Potier, I.; Taverna, M.; Fattal, E.; Benzaki, J.; Chevalier, M.; Ferrier, D. Performance evaluation of capillary surface treatments for peptide mapping by capillary zone electrophoresis, Chromatographia 2001, 53, 563-570.

(35) Ro, K. W.; Chang, W.-J.; Kim, H.; Koo, Y.-M.; Hahn, J. H. Capillary electrochromatography and preconcentration of neutral compounds on poly(dimethylsiloxane) microchips, Electrophoresis 2003, 24, 3253-3259.

(36) Pinto, D. M.; Ning, Y.; Figeys, D. An enhanced microfluidic chip coupled to an electrospray Qstar mass spectrometer for protein identification, Electrophoresis 2000, 21, 181-190.

(37) Katayama, H.; Ishihama, Y.; Asakawa, N. Stable Cationic Capillary Coating with Successive Multiple Ionic Polymer Layers for Capillary Electrophoresis, Anal. Chem. 1998, 70, 5272-5277.

(38) Chen, J.; Wabuyele, M.; Chen, H.; Patterson, D.; Hupert, M.; Shadpour, H.; Nikitopoulos, D.; Soper, S. A. Electrokinetically Synchronized Polymerase Chain Reaction Microchip Fabricated in Polycarbonate, Anal. Chem. 2005, 77, 658-666.

(39) Reschke, B. R.; Schiffbauer, J.; Edwards, B. F.; Timperman, A. T. Simultaneous separation and detection of cations and anions on a microfluidic device with suppressed electroosmotic flow and a single injection point, Analyst 2010, DOl:10.1039/b921914e.

(40) Kim, S. J.; Wang, Y.-C.; Lee, J. H.; Jang, H.; Han, J. Concentration polarization and nonlinear electrokinetic flow near a nanofluidic channel, Physical Review Letters 2007, 99, 044501/044501-044501/044504. 


\section{Chapter 6}

\section{Future Work}

While significant strides have been made toward the development and understanding of NMIs for use as analyte concentrators through the work presented here, many improvements are still needed for analyte concentrators to be used in the routine analyses of real-world proteomic samples. A few main issues exist including device stability, protein solubility, and protein identification.

The stability and reproducibility of the devices needs to be improved for use with real samples. The devices do not always reach and maintain the increased enrichment over time, which is problematic for analysis of real samples. For the analysis of real samples, the amount and rate of enrichment must be predictable based on multiple experiments. If the amount and rate of enrichment are not reproducible, then it will be difficult to determine an appropriate length of time to carry out the enrichment process for sample analysis. One aspect of these devices that must be improved is the nanochannels, which will enhance the reproducibility and stability of the devices.

To facilitate the fabrication of better nanochannels several desired characteristics must be realized. First, reproducible methods of creating nanochannels with similar sizes must be established. Second, the size of the nanochannel must be stable over the life of the device. Often, the nanochannels formed by dielectric breakdown in glass that integrate nanochannels would initially exhibit concentration polarization but their ability to develop concentration polarization would diminish during their use. Subsequent measurement of the nanochannel size indicated that it had increased in size as more experiments were carried out and compromised the performance of the device. The reproducibility of the fabrication and the stability of nanochannels used in this work are not reliable enough to use for real proteomic samples and therefore, better methods are needed.

To use these devices with real protein samples, the behavior of proteins during the enrichment process needs to be characterized. If the concentration of 
proteins becomes too high during the enrichment process, they have a tendency to precipitate. Therefore, optimization of the enrichment process is needed to reach a detectable level of analyte without leading to precipitation. Obviously, not every protein will behave identically; however, experiments with proteins known to precipitate easily could be carried out and compared to experiments using proteins that are easier to keep in solution. These experiments would help indicate how much enrichment is useful before it creates deleterious effects. Also, increased salt concentration can cause some proteins to precipitate. The behavior of the buffer ions is not completely understood, but it is believed that the concentration of the buffer increases in the local region where the fluorescent analyte concentration is increasing. Therefore, the concentration of the buffer could be varied to determine its influence on the precipitation of the proteins and therefore optimizing the buffer concentration.

The last major obstacle is the identification of the proteins. In this work, fluorescent imaging is used to characterize the enrichment process. Fluorescent imaging is not a viable option for real proteomic samples.

Proteomics usually involves attempting to identify several proteins and some of the proteins may be novel. Therefore, proper standards do not exist to compare to the unknowns in the sample. Second, proteins are typically not fluorescent at wavelengths compatible with fluorescence microscopes and therefore must be labeled in order to observe them using fluorescence. The efficiency of the labeling reaction would become a major issue and then the excess unreacted dye would have to be separated from the labeled proteins. This would add significant steps and sample handling that will decrease throughput and lead to sample contamination and loss. Therefore, mass spectrometry is the detection method that should be used, because it is capable of sequencing and identifying the proteins without a standard. This need requires that the analyte concentrator be coupled with mass spectrometry for real proteomic samples to be analyzed. 